\title{
Wideband and UWB Antennas for Wireless Applications: A Comprehensive Review
}

\author{
Renato Cicchetti, Emanuela Miozzi, and Orlandino Testa \\ Department of Information Engineering, Electronics and Telecommunications, University of Rome "La Sapienza", \\ Via Eudossiana 18, 00184 Rome, Italy \\ Correspondence should be addressed to Renato Cicchetti; cicchetti@die.uniromal.it
}

Received 22 July 2016; Revised 23 November 2016; Accepted 19 December 2016; Published 20 February 2017

Academic Editor: Ahmed Toaha Mobashsher

Copyright (C) 2017 Renato Cicchetti et al. This is an open access article distributed under the Creative Commons Attribution License, which permits unrestricted use, distribution, and reproduction in any medium, provided the original work is properly cited.

\begin{abstract}
A comprehensive review concerning the geometry, the manufacturing technologies, the materials, and the numerical techniques, adopted for the analysis and design of wideband and ultrawideband (UWB) antennas for wireless applications, is presented. Planar, printed, dielectric, and wearable antennas, achievable on laminate (rigid and flexible), and textile dielectric substrates are taken into account. The performances of small, low-profile, and dielectric resonator antennas are illustrated paying particular attention to the application areas concerning portable devices (mobile phones, tablets, glasses, laptops, wearable computers, etc.) and radio base stations. This information provides a guidance to the selection of the different antenna geometries in terms of bandwidth, gain, field polarization, time-domain response, dimensions, and materials useful for their realization and integration in modern communication systems.
\end{abstract}

\section{Introduction}

The antenna design for high-speed multimedia connectivity represents a challenging activity for designers of fixed and mobile wireless communication systems. In fact, the rapid growth of mobile systems toward the fifth-generation (5G systems) requires multiband, wideband, and UWB antennas suitable to cover mobile and wireless services and to reduce the system complexity, the overall device dimensions, and costs. Many efforts are underway to identify new antenna geometries suitable to satisfy the challenging requirements of the modern wireless communication systems [1-4]. This is particularly relevant in UWB applications operating in the extended frequency bands listed in Table 1, where the communication standards adopted by the major countries in the world are reported [5].

Planar, printed (2D profile), and 3D antennas have been developed with the aim of meeting the integration requirements and transportability of handheld devices (mobile phones, laptops, tablets, etc.) as well as those of radio base stations and vehicles (cars, airplanes, ships, etc.). In addition, antenna conformability may be a useful requirement to reduce the size and improve the look of mobile devices, while it results in an essential requirement for on-body type communication systems. This is particularly meaningful in wireless body area networks (WBANs) where high performances, as well as antenna flexibility under bending conditions, are important issues.

In general, 2D and 3D antenna technologies developed for wideband, multiband, and UWB applications can be divided into the following groups: microstrip antennas, monopole antennas over metal plates, printed monopole/dipole antennas, wide-slot antennas, metamaterial antennas, and dielectric resonator antennas (DRAs). These antennas may in some cases meet the demanding requirements of the existing communication systems, while they can be the starting point from which to develop new radiating systems suitable for the future communication requirements. To this end, in this paper a comprehensive review of the scientific literature of the last decade concerning these aspects is taken into account with the aim to provide researchers and designers with a valuable support tool to the antenna design. Particular emphasis is given to geometries, radiation mechanisms, broadbanding methods, materials, and prediction numerical tools adopted 
TABLE 1: UWB standards.

\begin{tabular}{|c|c|c|}
\hline Country & Frequency bands & Regulated by \\
\hline America, Canada & $\begin{array}{l}3.1-10.6 \mathrm{GHz} \\
\text { unlicensed band } \\
\text { without restrictions }\end{array}$ & $\begin{array}{l}\text { Federal } \\
\text { Communication } \\
\text { Commission (FCC) }\end{array}$ \\
\hline Europe & $\begin{array}{l}\text { 3.1-4.8 GHz with } \\
\text { Detect And Avoid } \\
\text { (DAA) restrictions; } \\
6-8.5 \mathrm{GHz} \text { band with } \\
\text { no restrictions }\end{array}$ & $\begin{array}{l}\text { Electronic } \\
\text { Communications } \\
\text { Committee (ECC) }\end{array}$ \\
\hline Japan & $\begin{array}{l}\text { 3.4-4.8 GHz with } \\
\text { DAA restrictions; } \\
7.25-10.25 \mathrm{GHz} \\
\text { unlicensed band }\end{array}$ & $\begin{array}{l}\text { Ministry of Internal } \\
\text { Affairs \& } \\
\text { Communications } \\
\text { (MICs) }\end{array}$ \\
\hline Korea & $\begin{array}{l}\text { 3.1-4.8 GHz with } \\
\text { DAA restrictions; } \\
7.2-10.2 \mathrm{GHz} \text { band } \\
\text { with no restrictions }\end{array}$ & $\begin{array}{l}\text { Electronics and } \\
\text { Telecommunications } \\
\text { Research Institute } \\
\text { (ETRI) }\end{array}$ \\
\hline Singapore & $\begin{array}{l}\text { 6-9 GHz band with } \\
\text { no restrictions; } \\
3.4-4.2 \mathrm{GHz} \text { band } \\
\text { with DAA restrictions }\end{array}$ & $\begin{array}{l}\text { Infocomm } \\
\text { Development } \\
\text { Authority (IDA) }\end{array}$ \\
\hline
\end{tabular}

to the analysis and design of these important classes of antennas.

The paper is organized in 12 sections. In Section 2 the features of some wideband microstrip antennas are presented. In Section 3, the characteristics of monopole antennas over metal plates are discussed. In Sections 4, 5, and 6, the performances of planar printed monopole, wide-slot, and dipole antennas for wideband and UWB applications are illustrated. In Section 7, directional UWB antennas are presented, in Section 8 metamaterial antennas are discussed, and in Section 9 antennas and materials useful to realize wearable antennas are considered, while in Section 10 the most recent advances of DRA antennas are illustrated. The main techniques adopted to realize UWB antennas with notched bands are highlighted in Section 11. Finally, in Section 12 some remarks concerning the state of the art, the future trends, and the main limitations of the different antenna manufacturing technologies being considered are outlined.

\section{Wideband Planar Microstrip Antennas}

Microstrip patch antennas (MPAs) were first introduced in the 1950s [6] but they found success only in the 1970s, driven by the fast growth of integration technology with active devices which allows realizing very compact structures. However, although these antennas exhibit low-profile, small size, limited manufacturing costs, and easy circuital integration, they typically present low fractional bandwidths $(\mathrm{FBW}=7 \%)$ not compatible with the modern wireless communication systems. To overcome this drawback, a significant research activity has been performed in the last twenty years to identify new geometries suitable to satisfy those requirements. This required the identification of the physical mechanisms governing the radiative processes taking place in said antennas.

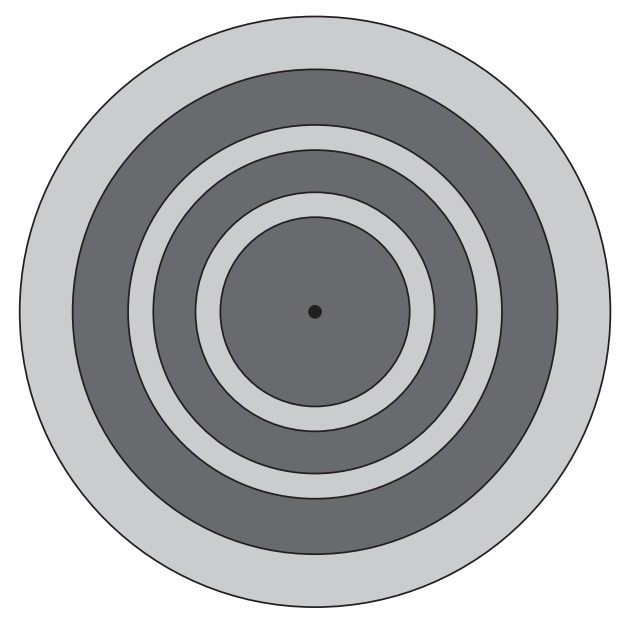

FIGURE 1: A monopolar patch antenna with a circular patch, circular ground plane, and two coupled metal rings [7]. The dot point indicates the coaxial probe pin. Dark gray monopole and light gray dielectric substrate.

In this section, some examples of the most recent antennas belonging to this important class are reported.

As a first structure, a wideband patch antenna having a radiation pattern similar to that of a monopole and consisting of two metal rings coupled to a circular patch radiator, presented in [7] is illustrated. The antenna is printed on a circular grounded dielectric substrate (DiClad 527), with relative permittivity $\varepsilon_{r}=2.5$, thickness of $1.5 \mathrm{~mm}$, and diameter $120 \mathrm{~mm}$, while a coaxial probe, located at the center of the circular patch, is used to excite the antenna (see Figure 1). The antenna has been analyzed using the full-wave FEM-based HFSS [8]. The antenna return loss is principally affected by the distances between the rings, between the first ring and the central radiating element, and by the radius of circular metal strip. The monopole-like radiation characteristics of the pattern are due to the central position of the excitation probe as well as to the symmetric field configurations excited in each slot through which the emission of electromagnetic energy occurs. The measured impedance bandwidth covers the 5.45-7.16 GHz frequency band (FBW $=27.1 \%$ ) making the antenna useful to be employed for wireless communication standards (WLAN, WiMAX). The realized measured peak gain ranges between $1.9 \mathrm{dBi}$ and $6.3 \mathrm{dBi}$, while the crosspolarization levels are of about $15 \mathrm{~dB}$ and $20 \mathrm{~dB}$ in the $H$-plane and $E$-plane, respectively.

A different design solution for a broadside coverage, consisting of a compact perturbed E-shaped patch antenna printed on a grounded dielectric substrate RT/duroid 5880 of dimensions $42 \mathrm{~mm} \times 42 \mathrm{~mm} \times 3.15 \mathrm{~mm}$ (about $0.82 \lambda_{0} \times$ $0.82 \lambda_{0} \times 0.0612 \lambda_{0}$, where $\lambda_{0}$ is the free-space wavelength at the antenna central frequency $f=5.38 \mathrm{GHz}$ ) and dielectric permittivity $\varepsilon_{r}=2.2$, has been presented in [9]. Two zigzag slots and two rectangular notches have been inserted in the patch for achieving a wider impedance bandwidth with higher return loss levels. To excite the antenna, a $50 \Omega$ probe is inserted between the first teeth of the zig-zag slots (see Figure 2). The zig-zag slots extend the current paths on the 


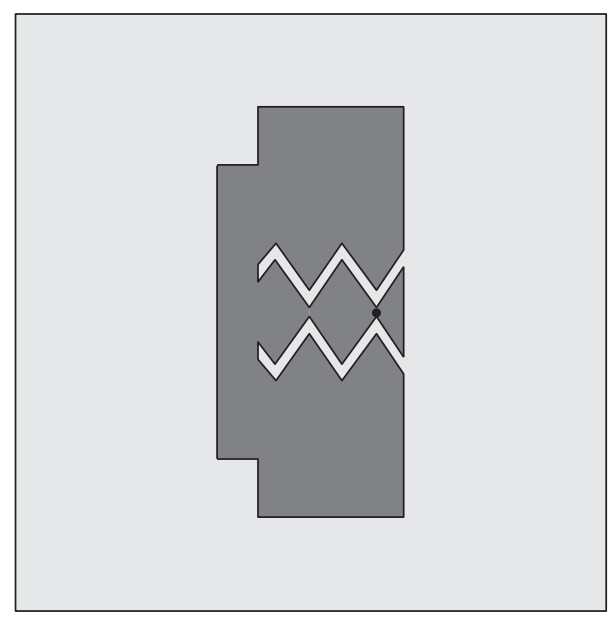

Figure 2: A compact perturbed E-shaped patch antenna [9]. Corner notches and zig-zag slots realized on the metallic patch are adopted to obtain a wideband behavior, while a $50 \Omega$ probe is inserted between the first teeth of the zig-zag slots to excite the antenna. Dark gray monopole and light gray dielectric substrate.

metal patch making the antenna less longer along the teeth direction reducing in this way the antenna dimensions. In addition, they excite two low $\mathrm{Q}$ resonant modes, resulting in an impedance antenna bandwidth ranging between $4.96 \mathrm{GHz}$ and $6.69 \mathrm{GHz}(\mathrm{FBW}=30 \%)$, thus ensuring the coverage of the Hiperlan/2, IEEE 802.11a, and HiSWaNa frequency bands. A homemade numerical prediction tool based on a locally conformal finite-difference time-domain (FDTD) scheme, together with the surface equivalence theorem employing the free-space dyadic Green's functions, has been adopted to compute the field distributions near and outside the FDTD computational domain [10]. At lower frequency the radiation patterns are similar to that of a simple rectangular patch antenna, while at higher frequencies they show a tilt of about $16.2^{\circ}$ from the boresight direction in the $E$-plane. This behavior can be explained with the presence of a surface current density traveling along the zig-zag region. Front-toback ratios are higher than $21 \mathrm{~dB}$ due to the adopted full ground solution, while the realized gains are between 4 and $7 \mathrm{dBi}$. An equivalent circuit useful to simplify the design of the RF front end and an analysis of the radiation mechanisms responsible for the antenna behavior are reported in [11].

To improve the impedance bandwidth a U-shaped antenna with a defect ground plane, adopted to reduce the reactive energy stored within the antenna cavity and in the neighboring region of the metal patch, has been proposed in [12]. Said antenna, useful for wideband wireless communications, is printed on a glass polytetrafluoroethylene (PTFE) dielectric substrate of permittivity $\varepsilon_{r}=2.4$, loss tangent 0.00022 , and thickness of about $1.6 \mathrm{~mm}$. A coaxial probe, placed in the antenna symmetry plane, is adopted to excite the antenna (see Figure 3). The effects of circular and square ground planes have been investigated. It is observed that antennas equipped with circular ground planes exhibit wider impedance bandwidth with respect to those equipped with a square ground plane having comparable dimensions. In particular, the antenna having a diameter of $36 \mathrm{~mm}$ offers the best radiative performance and covers the frequency range from $4.5 \mathrm{GHz}$ to $11.4 \mathrm{GHz}(\mathrm{FBW}=86.79 \%)$. Parametric optimizations have been performed using the full-wave commercial software Ansoft based on the Method of Moments. The measured gain ranges between $2.2 \mathrm{dBi}$ and $4.1 \mathrm{dBi}$ over the entire frequency band.

Other examples of wideband planar microstrip antennas can be found in [13-16].

\section{Monopole Antennas over Metal Plates (3D Profile)}

In 1976, Dubost and Zisler [17] introduced for the first time wideband monopole antennas arranged orthogonally on a metal ground plane. Even though their vertical size can prevent their integration into handset devices, different kinds of planar monopole antennas have been developed in the last twenty years, due to their several practical advantages. In particular, the metal plate can be usefully employed to increase the antenna gain and the front-to-back ratio, thus allowing obtaining a high electrical insulation with the RF circuitry of the communication system and preserving the antenna characteristics regardless of the installation site features [18]. Of particular practical interest are the monopoles or the folded antennas as they can be employed in base stations, avionics or automotive systems, and so forth. Said applications, which generally do not need compact communication systems, require instead low vertical-profile radiators when they are intended to be installed on highspeed mobile vehicles. A wide class of said antennas having the above-mentioned features have been proposed in the literature in order to obtain high radiative performance (see Figure 4). For the sake of brevity in this section an overview of the most significant results published in literature is provided.

Empirical formulas, to identify the frequency band covered by monopole antennas vertically placed on ground planes, useful during the preliminary design stage, are proposed in [19-22]. These formulas are derived assuming that the electromagnetic field, first to be radiated by the monopole, substantially propagates in the spatial region located between the lower edge of the monopole and the ground plane; that is, it experiences a propagation similar to that which occurs in a simple or tapered transmission line.

Adopting the lower profile of the monopole similar to that of a tapered transmission line, a planar inverted cone antenna (PICA) for broadband wireless applications has been proposed in [23]. Two different design solutions, intended for UWB applications related to imaging and communication, are considered (see Figure 5). In particular, the first design solution operates in the frequency range between 1 and $9 \mathrm{GHz}$, while the second one, provided with holes, covers between 1 and more than $10 \mathrm{GHz}$ with a FBW $=163 \%$. Both antennas are characterized by an adequate level of crosspolarization and frequency stability of the radiation diagrams (best performances are exhibited by the solution provided with holes) up to about $7 \mathrm{GHz}$. The antenna gain varies between 4 and $8 \mathrm{dBi}$ in the frequency range between 1 and $10 \mathrm{GHz}$. The monopole, of length of about $76.2 \mathrm{~mm}\left(\lambda_{0} / 4\right.$, 


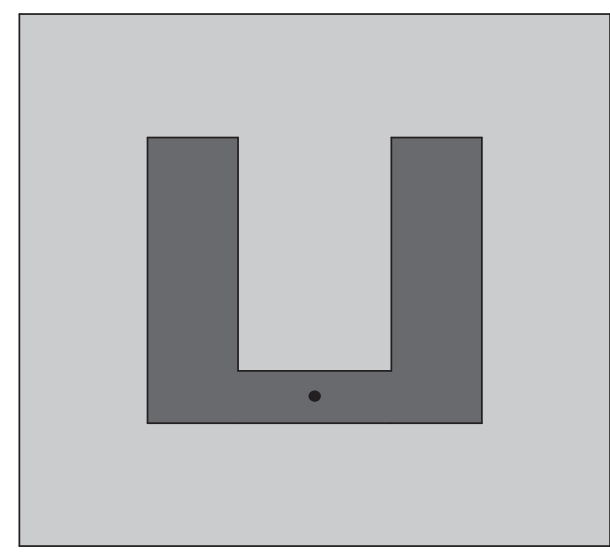

(a)

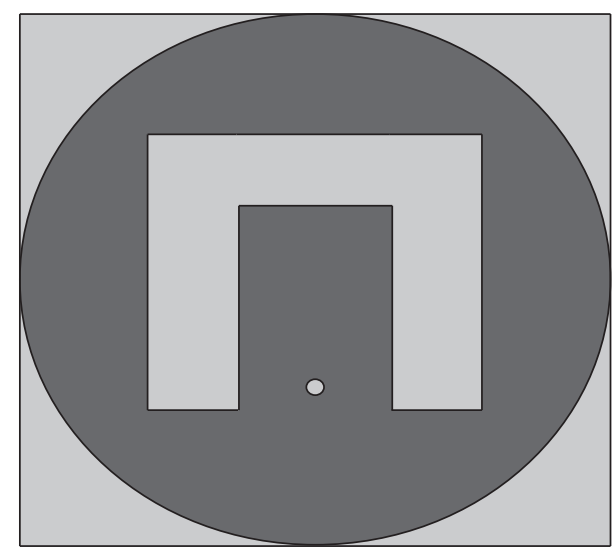

(b)

FIGURE 3: U-shaped patch antenna with a U-slot embedded in a circular/square ground plane [12]. Top view (a) and bottom view (b). The black dot point indicates the pin of the coaxial probe. Dark gray monopole and light gray dielectric substrate.

where $\lambda_{0}$ is wavelength at the lower working frequency), is printed on a substrate of relative dielectric permittivity 2.33 and thickness $0.79 \mathrm{~mm}$, while the metal plate has dimensions of $610 \mathrm{~mm} \times 610 \mathrm{~mm}\left(2 \lambda_{0} \times 2 \lambda_{0}\right)$. The structure, fed by a coaxial probe, is spaced from the ground plane of $0.64 \mathrm{~mm}$ to increase the operating band. The antenna was analyzed and designed using a homemade software based on the full-wave FDTD numerical technique.

A similar design solution, but having a wider bandwidth, has been proposed in [24]. This solution adopts a leaf-shaped monopole having three circular holes, mounted vertically on a grounded dielectric slab of dimensions $80 \mathrm{~mm} \times 80 \mathrm{~mm}$, with dielectric constant $\varepsilon_{r}=3.5$ and thickness of $1.5 \mathrm{~mm}$ (see Figure 6). The holed monopole, of height $76.2 \mathrm{~mm}$ and thickness $1 \mathrm{~mm}$, together with an air gap of $0.64 \mathrm{~mm}$, is employed to improve the antenna bandwidth that extends from $1.3 \mathrm{GHz}$ up to $29.7 \mathrm{GHz}(\mathrm{FBW}=183 \%)$. The antenna gain ranges between 3.6 and $4.7 \mathrm{dBi}$ in the frequency band between 3 and $9 \mathrm{GHz}$. The applications for which the antenna is proposed are of commercial and military nature. The antenna was analyzed by means of the full-wave FIT-based CST Microwave Studio [25]. The measurement results are in good agreement with the numerical simulations.

Simple monopoles, with bevels and with vertical foldings of the radiating surfaces, for broadband and UWB applications, have been analyzed in [20]. Both structures, vertically mounted on a ground plane of dimensions $260 \mathrm{~mm} \times 260 \mathrm{~mm}$, have a height of $45 \mathrm{~mm}$ and an air gap of $1 \mathrm{~mm}$, while the bevel angle is of $16^{\circ}$ for the monopole and $20^{\circ}$ for the accordion version. In particular, it is shown how both the monopole tapering and the bending are able to expand significantly the antenna operating band (see Figure 7). In fact, the adoption of the two mentioned techniques not only widens the antenna bandwidth but makes the radiation patterns more stable in frequency. The performances of the antennas were analyzed by means of the full-wave FIT-based CST Microwave Studio [25]. The results of the measurements performed on the antenna prototypes are found to be in good agreement with the numerical computations.
A modified bevel technique is adopted in [26], where two monopole antennas suitable to cover the AMPS (824$894 \mathrm{MHz}$ ), the GSM900 (890-960 MHz), and the DCS (1710$1880 \mathrm{MHz}$ ) frequency bands were presented. The antennas are proposed for multiband mobile communication systems such as receivers for vehicle applications. A homemade fullwave method of moment (MoM), based on the Rao-WiltonGlisson (CRWG) basis functions, has been employed to solve the electric field integral equation (EFIE) from which the antenna surface current distributions have been derived. The radiating element, of dimensions of $50 \mathrm{~mm} \times 87 \mathrm{~mm}$ and thickness of $0.5 \mathrm{~mm}$, with bevel cuts on its two lower corners, is connected to a $50 \Omega$ SMA connector pin. The first radiant structure, composed of a rectangular monopole having a bevel angle of $22.3^{\circ}$, a suitable width of the feeding strip, and a gap distance of $3 \mathrm{~mm}$ from the ground plane, is depicted in Figure $8(\mathrm{a})$. The top edge of the radiating element is rounded to improve the high-frequency performance of the monopole. The geometrical parameters affecting the antenna bandwidth are the height of the monopole, which controls the lower frequency, and the bevel angle which controls the antenna upper frequency. In this way, an antenna whose gain varies from 4 to $7 \mathrm{dBi}$ is obtained.

The second monopole antenna, having a staircase profile of its contour (see Figure 8(b)), presents a larger tapering region close to the feeding strip. The monopole of dimensions $68 \mathrm{~mm} \times 69.5 \mathrm{~mm}$, presenting an air gap of $1.8 \mathrm{~mm}$, is placed vertically on a ground plane of size $500 \mathrm{~mm} \times 500 \mathrm{~mm}$. The radiation diagrams show a frequency behavior similar to those of the first monopole antenna even if the presence of a staircase profile introduces some ripples in the radiation diagrams, while the gain increases monotonically from about 2.5 up to $6.3 \mathrm{dBi}$ in the frequency range of $800-1880 \mathrm{MHz}$. Note that the total height of the second antenna is only of $0.19 \lambda_{L}$, which is much lower than the height of the first antenna $0.24 \lambda_{L}\left(\lambda_{L}\right.$ is the wavelength at the lower edge of the AMPS frequency band).

A combined technique to realize a wideband antenna, consisting of a rectangular monopole with a bevel and 

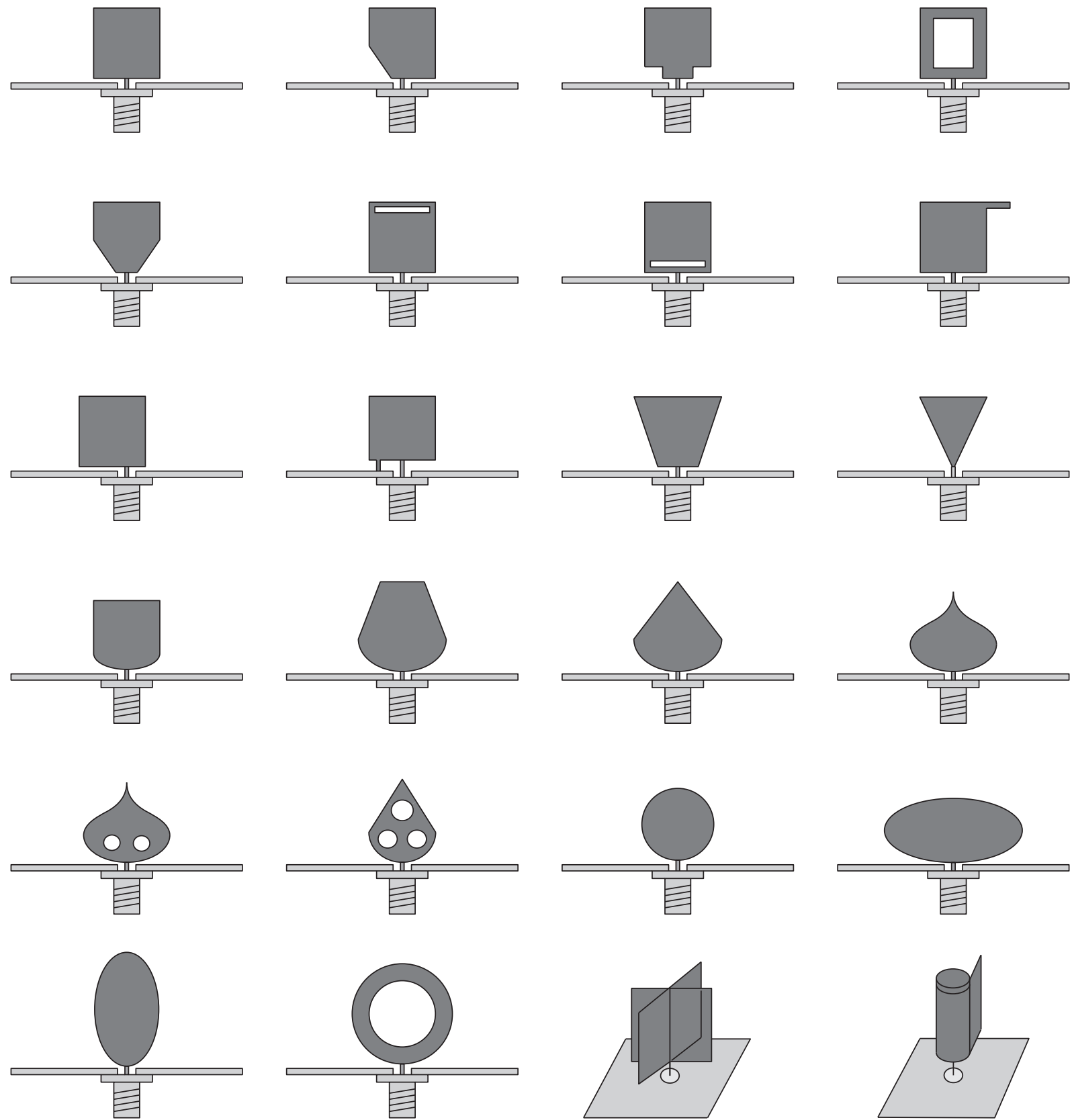

FIGURE 4: Examples of broadband and UWB monopole antennas over a metal plate. Dark gray monopole, medium gray ground plane, and light gray dielectric substrate.

a shorting post, has been proposed in [27]. The antenna exhibits a frequency band with a VSWR $\leq 3$ ranging from $800 \mathrm{MHz}$ to more than $10.5 \mathrm{GHz}$. Said antenna (see Figure 9) can meet the requirements of the GSM, PCS, IMT-2000, and DECT of the $2.4 \mathrm{GHz}$ and $5.8 \mathrm{GHz}$ ISM bands, U-NII, as well as those of the most recent UWB communication systems. The frequency behavior of the parameter VSWR was simulated by means of a homemade software based on the MoM technique, using a finite-gap voltage-feed source and a wire-grid mesh with piecewise sinusoidal basis functions. The experimental results performed on an antenna prototype show that the simultaneous adoption of the short circuit strip and of the bevel (whose angle should not exceed $40^{\circ}$ ) realized in the lower edge profile of the monopole is able to increase significantly the antenna bandwidth. The monopole has a size of $60 \mathrm{~mm} \times 60 \mathrm{~mm} \times 0.2 \mathrm{~mm}$, with a feed gap of $1.2 \mathrm{~mm}$ and a shorting post of $2 \mathrm{~mm}$, while the ground plane has a size of approximately $200 \mathrm{~mm} \times 200 \mathrm{~mm}$.

An excitation system with three branches named tridentshaped feeding line, proposed to obtain an enhancement of the antenna bandwidth and a better polarization purity of the radiated field, has been presented in [28]. The antenna consists of a squared monopole (size $40 \mathrm{~mm} \times 40 \mathrm{~mm} \times$ $0.2 \mathrm{~mm}$ ) placed vertically with respect to a ground plane 


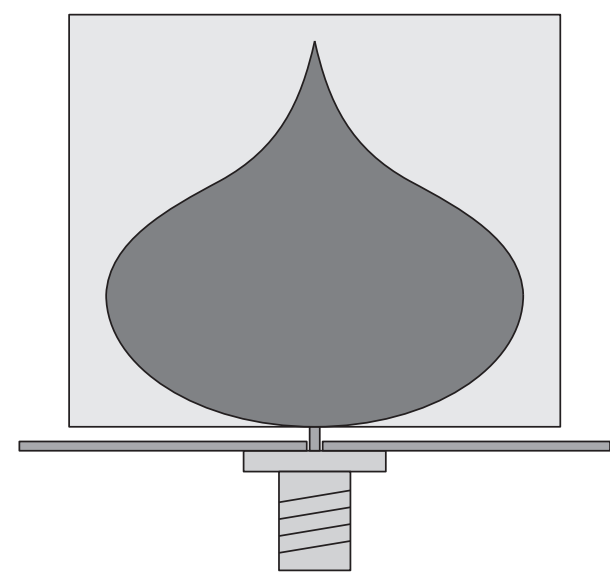

(a)

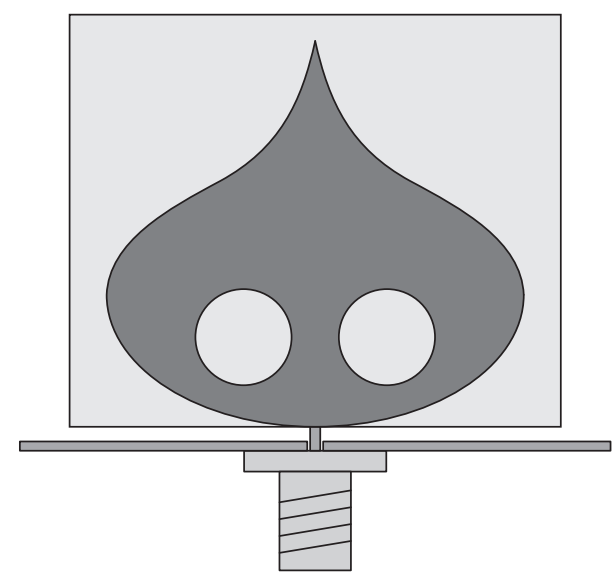

(b)

Figure 5: The PICA broadband antenna [23]. The design solutions (a) without holes and (b) with holes in the monopole are shown in the figure. Dark gray monopole and medium gray ground plane.

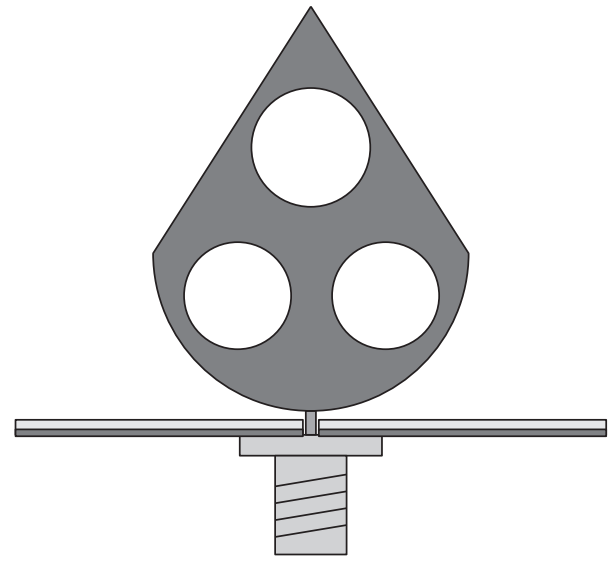

FIgURE 6: The leaf-shaped antenna [24]. The holes in the monopole are used to modify the surface currents distribution giving rise to a more favorable reactive energy configuration near the monopole. Dark gray monopole and medium gray ground plane.

of dimensions $150 \mathrm{~mm} \times 150 \mathrm{~mm}$. The improvement of the antenna characteristics is obtained thanks to a better spatial distribution of the vertical component of the surface current together with a drastic reduction of the transverse component excited on the monopole (see Figure 10). The experimental measurements confirm that the monopole exhibits a narrowband behavior when it is excited by a single feed point, while its behavior becomes broadband, with a frequency band ranging between 1.4 and about $11.5 \mathrm{GHz}$, when the monopole is fed in three equal-spaced points. The antenna is proposed for the applications concerning the new broadband wireless metropolitan area network system using the IEEE 802.16a (2$11 \mathrm{GHz}$ ) standard [29]. The antenna gain varies from about 4 to $7 \mathrm{dBi}$, in the frequency range between about 1.3 and $6 \mathrm{GHz}$, while maintaining fairly constant in the frequency range between $6 \mathrm{GHz}$ and $10 \mathrm{GHz}$. The simulated results are obtained using the full-wave FEM-based HFSS [8].
A technique useful to improve the impedance bandwidth of a rectangular monopole antenna, consisting in the use of an additional rectangular metallization, placed orthogonal with respect to the monopole axis, so as to obtain a resulting crossshaped geometry, having the aim of obtaining an antenna suitable to cover the UWB band (3.1-10.6 GHz), has been proposed in [30]. The monopole of dimensions $24 \mathrm{~mm} \times$ $18 \mathrm{~mm}$ is placed vertically on a circular metal plate (see Figure 11) whose diameter is of about $150 \mathrm{~mm}$ (corresponding to about $3.2 \lambda_{0}$, where $\lambda_{0}$ is the free-space wavelength relative to the central working frequency). The antenna gain ranges between about 3.1 and $5.8 \mathrm{dBi}$. The cross-polarization levels are similar to those of a rectangular monopole. The radiation phenomena occur near the lower edge of the monopole. The full-wave FIT-based CST Microwave Studio [25] and MoMbased WIPL-D [31] are used for the numerical simulations.

Finally, an example of a wideband antennas array is reported. The array is formed by four horizontal pinwheelshaped folded monopole planar antennas (PMAs) assembled with additional four vertical PMAs to improve the directivity and the performance of the circular polarization [32]. Since the overall antenna height is of $19 \mathrm{~mm}$ it can be easily integrated into low-profile devices. Moreover, supporting the circular polarization it may be useful to realize radio base stations for indoor applications. The antenna has an impedance bandwidth ranging between 1.05 and $2.55 \mathrm{GHz}(\mathrm{FBW}=87 \%)$ in which the axial ratio $\mathrm{AR}<3 \mathrm{~dB}$. The antenna is realized on an RF-35 substrate with a dielectric constant of 3.5 and thickness of $0.5 \mathrm{~mm}$, having a ground plane of $150 \mathrm{~mm} \times$ $150 \mathrm{~mm}$. The PMAs are excited by means of strip fed with bevels, used to increase the antenna bandwidth, connected to a planar balun formed by a broadband Wilkinson power divider and by a commercial hybrid coupler. The length of the folded part of the monopole, as well as its distance from the ground plane, determines the in-band entrance, while the width of each radiating element controls the array bandwidth. An excitation with increasing phase step of $90^{\circ}$ between adjacent monopoles ensures the excitation of the circular 


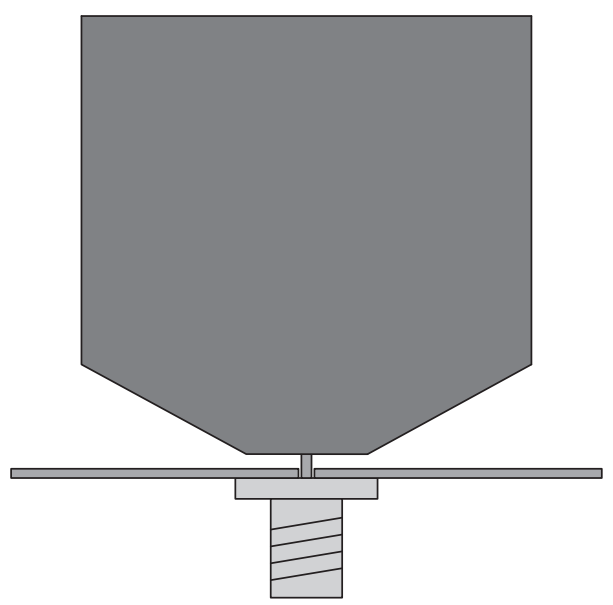

(a)

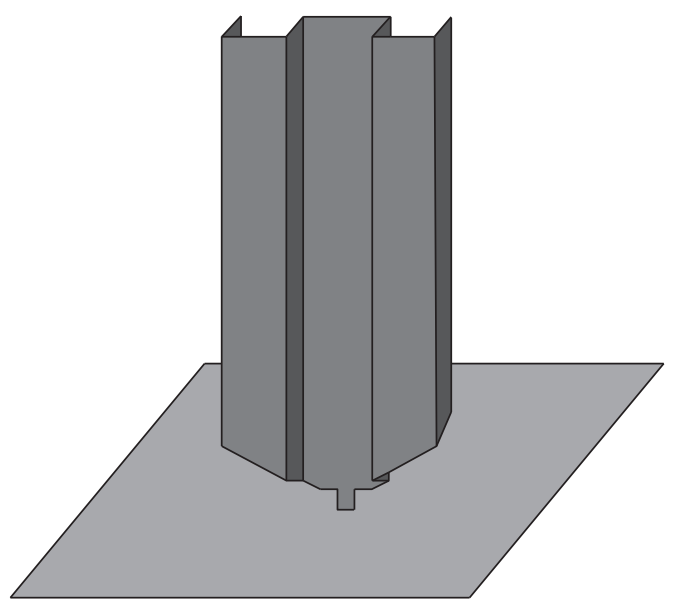

(b)

FIGURE 7: Hexagonal monopole antenna (a) and folded monopole antenna (b) for broadband and UWB applications [20]. The accordion version is adopted to reduce the transverse size of the monopole. Dark gray monopole and medium gray ground plane.

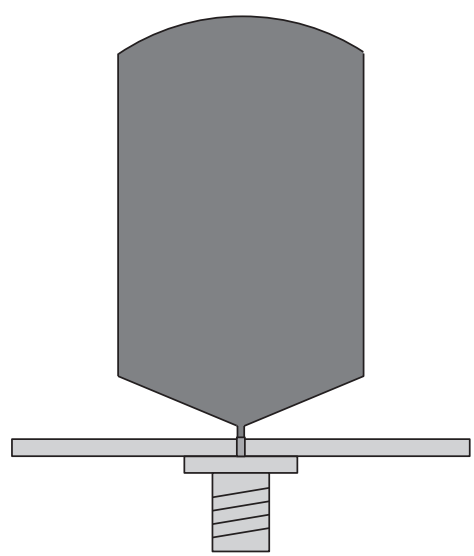

(a)

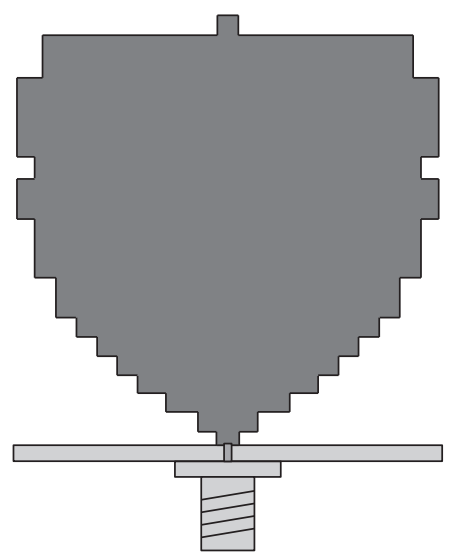

(b)

FIGURE 8: Monopole antenna (a) with bevels and (b) with staircase contours [26]. The bevels or the staircase contours are used to facilitate the energy emission in the monopole region near the ground plane. Dark gray monopole and medium gray ground plane.

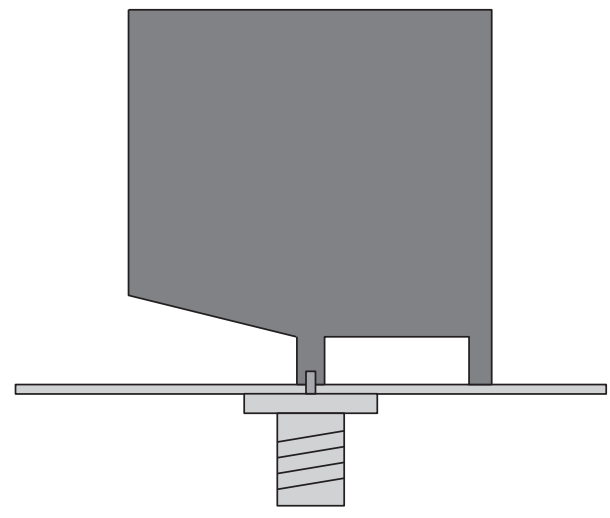

FIGURE 9: A rectangular monopole with a shorting post. Dark gray monopole and medium gray ground plane [27].

polarization along the boresight direction. The resulting antenna gain is of about $8.24 \mathrm{dBi}$. Two frequency notches are introduced to avoid the energy emission at the frequencies of 2.03 and $2.39 \mathrm{GHz}$. This result is achieved through the adoption of a U-shaped resonant $\operatorname{strip}(f=2.03 \mathrm{GHz})$ and an inverted $\mathrm{W}$-shaped resonant slot $(f=2.39 \mathrm{GHz})$. The electromagnetic analysis was performed using the full-wave FIT-based CST Microwave Studio [25].

\section{Printed Monopole Antennas (2D Profile)}

Modern portable devices, working with high data rates, employ broadband antennas characterized by small sizes and low profiles. In fact, whereas monopoles antennas, having a tridimensional shape, are more suitable to be used in radio base stations and mobile radio systems (cars, trains, airplanes, ships, etc.), the printed monopole antennas not only reduce the vertical bulk of the portable device, but also enable an easy integration with the RF circuitry. Notice that typical smart phones, tablets, and laptops exhibit size of about $130 \mathrm{~mm} \times 50 \mathrm{~mm} \times 6 \mathrm{~mm}, 240 \mathrm{~mm} \times 140 \mathrm{~mm} \times$ 


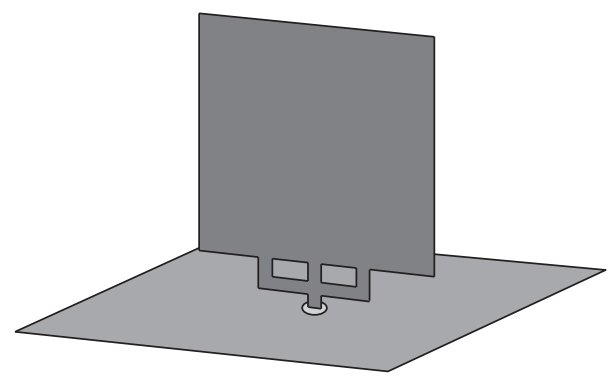

FIgURE 10: A planar metal plate monopole antenna employing a trident-shaped feeding network [28]. The multipoint excitation is used to increase the amplitude of vertical component of the surface current excited in the monopole. Dark gray monopole and medium gray ground plane.

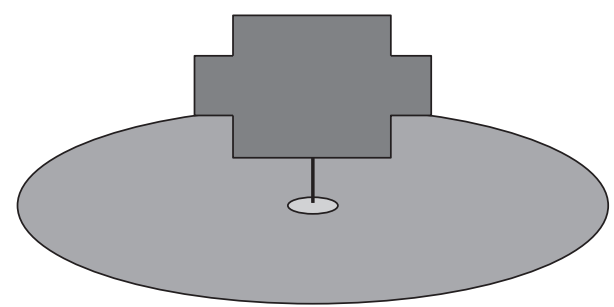

Figure 11: A cross-shaped monopole antenna [30]. The additional metal plate, placed orthogonal with respect to the main rectangular metallization, is employed to reduce the field discontinuity caused by the transition between the coaxial probe and the radiant monopole, resulting in a wideband antenna behavior. Dark gray monopole, medium gray ground plane, and light gray dielectric substrate.

$8 \mathrm{~mm}$, and $260 \mathrm{~mm} \times 200 \mathrm{~mm} \times 21 \mathrm{~mm}$, respectively. The need to satisfy these important requirements has given rise to a strong research activity that led to the development of a large class of antennas. In this context, of particular interest are the wideband [33-38], the multiband [39-48], and the UWB printed monopole antennas, simple [49-57] and with notched bands to avoid interference with other radio communication systems [58-68] or those suitable for supporting the circular polarization [33, 69-71].

A large class of geometries (rectangular, circular, elliptical, triangular, hexagonal, annular, etc.) has been proposed in order to improve the impedance matching of said antennas with the feeding line, generally consisting of a microstrip line, simple or tapered $[40-42,51-57,62,69,72,73]$, or based on a coplanar waveguide (CPW) having a single or multiple transition $[34,35,39,60,71,74]$. Of particular interest are also the techniques based on the use of notches placed near the transition region between the ground plane and the radiating monopole $[33,36,72]$, on the use of trapezoidal $[35,72,74]$ or tapered ground planes $[49,58,59,63,68,75]$, having zig-zag shaping $[76,77]$, or with defects in the ground plane (DGS) $[34,37,39,62,78,79]$. Additional techniques are those based on the use of bevels $[37,80]$ or of a particular shape of the upper region of the monopole [81]. For the sake of brevity in the following an overview of the most significant papers concerning the aforementioned design aspects is provided.

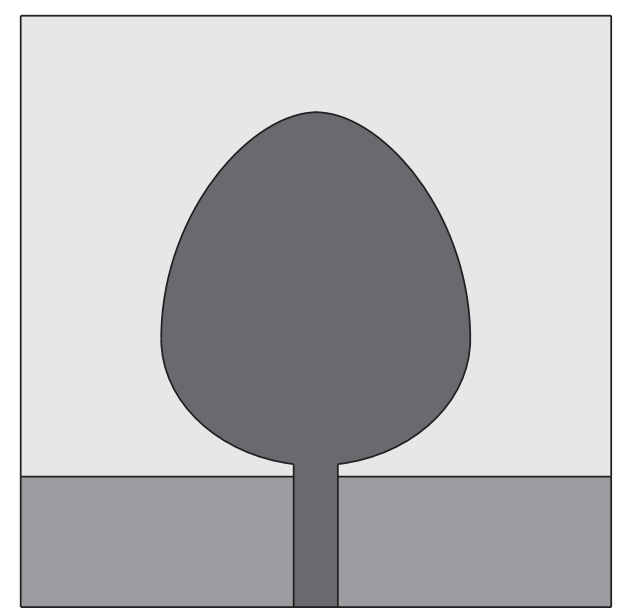

FIGURE 12: The printed Newton's egg curved monopole antenna [51]. The regular contour expressed by Newton's egg curve promotes the energy emission in the region close to the ground plane avoiding energy reflections toward the feeding line. Dark gray upper metal face, medium gray bottom metal face, and light gray dielectric substrate.

4.1. UWB Antennas. The UWB antennas are characterized by extremely high operating bands. In these bands the antennas have to ensure a low signal distortion as well as an adequate stability of the group delay, of the antenna gain, and of the angular behavior of the radiation patterns [82]. To this end, in this section, UWB antennas and suitable design solutions useful to improve the antenna bandwidth are presented.

A suitable low-profile geometry useful to radiate UWB signals is Newton's egg curved monopole antenna [51]. A prototype of said antenna (see Figure 12), of dimensions $40 \mathrm{~mm} \times$ $40 \mathrm{~mm}$, is printed on double-sided FR-4 substrate with relative permittivity $\varepsilon_{r}=4.4$, loss tangent 0.018 , and thickness $h=$ $1.6 \mathrm{~mm}$. A parametric formula, based on Newton's egg curve, is proposed for the design of the monopole profile, while an empirical formula is introduced to determine the lower cut-off frequency of the impedance bandwidth. The antenna analysis and design have been performed using the full-wave FEM-based HFSS [8]. The experimental measurements show that the antenna has a FBW of $116.55 \%(2.9-11.0 \mathrm{GHz})$ and exhibits a stable gain of about $2.2-2.9 \mathrm{dBi}$. The proposed antenna exhibits almost omnidirectional radiation patterns with low cross-polarization in all directions and adequate group delay over the entire UWB bandwidth $(3.1-10.6 \mathrm{GHz})$.

Three different UWB antenna geometries (see Figures 13 and 14) useful to transmit short pulse signals, and characterized by reduced overall dimensions, have been proposed in [52]. These antennas, having impedance bandwidth ranging from 3 up to $11 \mathrm{GHz}$, are realized using a Rogers $4350 \mathrm{~B}$ substrate having a dielectric permittivity of 3.48 and thickness $0.8 \mathrm{~mm}$. A thin substrate is used to limit the excitation of surface waves. In particular, the first antenna, of dimensions $30 \mathrm{~mm} \times 30 \mathrm{~mm}$, consists of an elliptic monopole excited by a tapered microstrip line and equipped with a trapezoidal ground plane. The second radiating structure, of dimensions $15 \mathrm{~mm} \times 30 \mathrm{~mm}$, is obtained by removing the left half part 


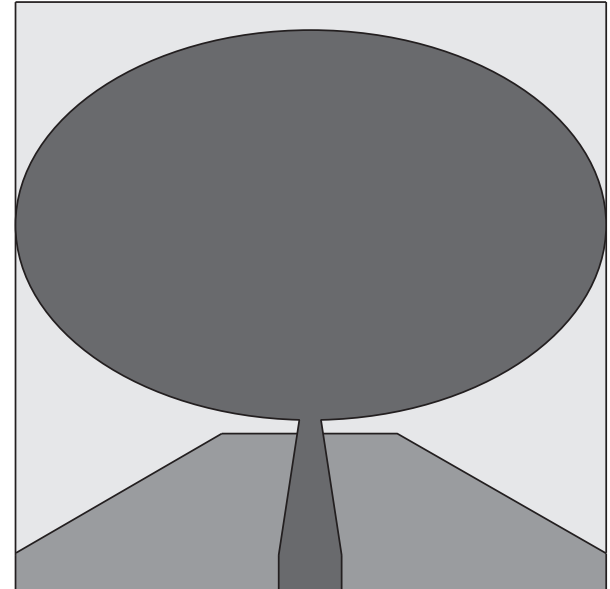

FIGURE 13: Configuration of the full-size UWB antenna [52]. Dark gray upper metal face, medium gray bottom metal face, and light gray dielectric substrate.

of the monopole, while the last geometry, of dimensions $12 \mathrm{~mm} \times 17 \mathrm{~mm}$, is derived by removing from said antenna, part of the upper and right side of the monopole and the lower part of the ground plane. Despite the considerable reduction of the antenna size observable in Figure 14(b), the antenna response to pulse signals having the waveform of the fifthderivative of the Gaussian pulse is excellent. Further information useful to reduce the size and increase the performance of symmetric UWB antennas can be found in [3].

4.2. Monopole Antennas for Circular Polarization. The printed monopole antennas typically work in linear polarization. This kind of field polarization although useful in many applications cannot be very effective when the communication systems have to operate in indoor environments where multipath and scattering processes can reduce the quality of the radio link [83]. To this end, several kinds of printed monopole antennas suitable to support a circular polarization have been recently proposed in the literature $[33,36,38,69-$ $71,84,85]$. In particular, in the papers discussed below the main features of some of them are reported.

A very simple monopole antenna, obtained by extending the feeding microstrip line beyond the ground plane in the upper left part region of which two notches were made thus leading to an asymmetry in the metal screen (see Figure 15), has been proposed in [33]. The electromagnetic characteristics of the antenna have been derived using the full-wave FEM-based HFSS [8]. The measured impedance bandwidth is of about $5.96 \mathrm{GHz}$ from 4.06 to $10.02 \mathrm{GHz}$, while the measured AR bandwidth is of about $2.64 \mathrm{GHz}$ from 5.91 to $8.55 \mathrm{GHz}$. The antenna, of dimensions of $16 \mathrm{~mm} \times$ $22 \mathrm{~mm} \times 1 \mathrm{~mm}$, is realized on a FR4 substrate with a dielectric permittivity of 4.6 and loss tangent of 0.02 . The circular polarization is induced by the asymmetrical ground plane, which is responsible for the excitation of both the horizontal and vertical component of the surface current with a phase shift of about $90^{\circ}$, while a notch placed under the monopole is used to increase the antenna impedance bandwidth. The radiation patterns are mainly left handed circularly polarized (LHCP) in the lower half-space and right handed circularly polarized (RHCP) in the upper half-space, while the cross-polarization can keep more than $15 \mathrm{~dB}$ lower than the copolarization in each plane. The antenna gain is between 1 and $2.8 \mathrm{dBi}$.

Similar radiation mechanisms are employed in the antenna proposed in [36] where a rectangular monopole, fed asymmetrically by means of a microstrip line and equipped with a ground plane integrating a metal stub and a slit, is adopted to obtain a circularly polarized electromagnetic field (see Figure 16). According to the measurement results, the antenna covers the frequency range between 2.32 and $8.88 \mathrm{GHz}$ with an impedance bandwidth of $6.56 \mathrm{GHz}$. AR values of less than $3 \mathrm{~dB}$ are observed in the frequency range between 3.2 and $4.4 \mathrm{GHz}$ (AR bandwidth of $1.2 \mathrm{GHz}$ ). The antenna, of dimensions of $45 \mathrm{~mm} \times 40 \mathrm{~mm} \times 1.6 \mathrm{~mm}$, is printed on a FR4 substrate with a relative permittivity $\varepsilon_{r}=$ 4.4 , loss tangent 0.024 , and thickness $1.6 \mathrm{~mm}$. The full-wave FEM-based HFSS [8] was adopted to analyze the antenna.

The slit is adopted to increase the frequency band where the radiated field is circularly polarized, while the stub is used to restore the antenna impedance bandwidth perturbed by the presence of the slit. The antenna gain ranges between 1 and $2 \mathrm{dBi}$, while the efficiency is of about $78 \%$.

A more complex design solution, consisting of a C-shaped monopole, coupled to an open metallic loop printed on the opposite face of a FR4 dielectric substrate with a relative permittivity of 4.4 , a loss tangent of 0.02 , and dimensions of $25 \mathrm{~mm} \times 25 \mathrm{~mm} \times 1 \mathrm{~mm}$, has been proposed in [38]. A rectangular stub, integrated into the upper edge of the ground plane, and coupled to the open metallic loop, is used to improve the impedance and the axial ratio (AR) bandwidth (see Figure 17). The gap in the loop significantly influences the bandwidth of the AR but not that of the impedance. The antenna, analyzed using the full-wave FEM-based HFSS [8], has an impedance bandwidth ranging between 4.26 and $10.92 \mathrm{GHz}(\mathrm{FBW}=87.7 \%)$ and an $\mathrm{AR}$ bandwidth ranging between 4.28 and $8.42 \mathrm{GHz}$ (AR FBW $=65.2 \%$ ). A solution equipped with a metal reflector, placed at a distance of about $16 \mathrm{~mm}$ from the antenna ground plane, is suggested to make unidirectional the radiation pattern and to increase the antenna gain of about $3 \mathrm{~dB}$ without affecting significantly the operating band of the antenna.

A different radiation mechanism is excited in the dualband circularly polarized antenna in [85] consisting of a rectangular metal frame, acting as a ground, and an Sshaped monopole working as a radiator. The antenna having dimensions $37 \mathrm{~mm} \times 37 \mathrm{~mm} \times 1 \mathrm{~mm}$ has been fabricated on a FR4 substrate with dielectric permittivity of 4.4 and loss tangent 0.02 (see Figure 18). The proposed antenna is capable of operating in the $2.39-2.81 \mathrm{GHz}$ and $5.42-5.92 \mathrm{GHz}$ bands, thus suitable for the WLAN and WiMAX applications. The full-wave FEM-based HFSS [8] has been adopted to determine the antenna characteristics. The circular polarization is obtained by means of a suitable design of the S-shaped monopole and of the external metallic frame. This frame 


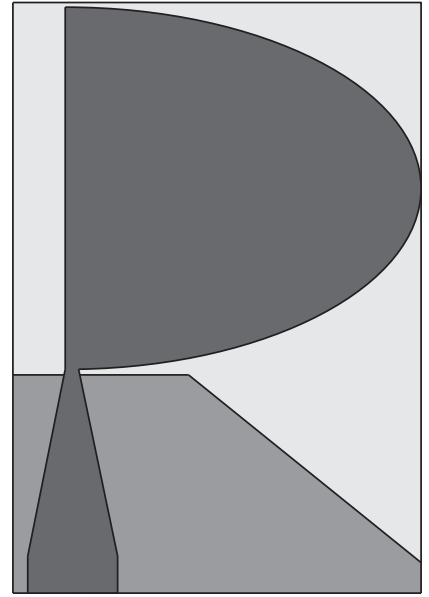

(a)

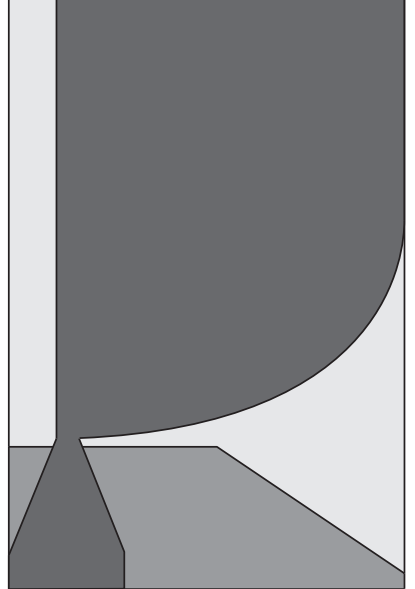

(b)

FIGURE 14: Configuration of the half-size (a) and of the compact UWB antenna (b) [52]. Dark gray upper metal face, medium gray bottom metal face, and light gray dielectric substrate.

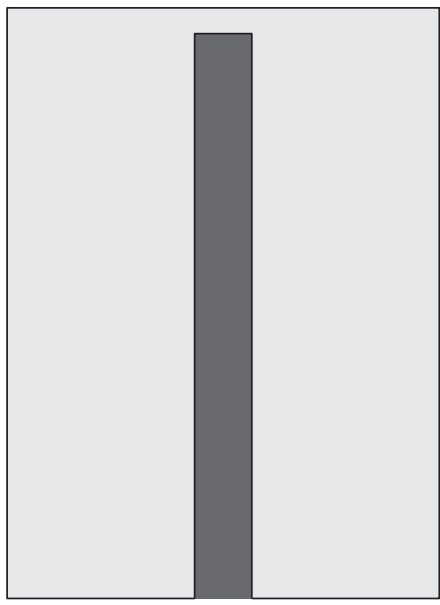

(a)

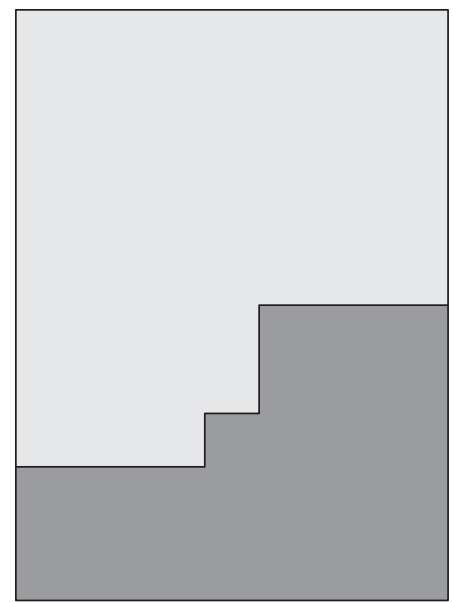

(b)

FIGURE 15: A planar monopole antenna with wideband circular polarization [33]. Dark gray upper metal face, medium gray bottom metal face, and light gray dielectric substrate. Top view (a) and bottom view (b).

determines the excitation of edge currents whose radiated field, along with that sustained by the monopole, is suitable to support a circular polarization in the band of interest. The radiation efficiency is approximately $80 \%$, while the gain ranges between 3.2 and $4 \mathrm{dBi}$.

Further papers concerning printed monopole antennas suitable to support the circular polarization can be found in [69-71].

4.3. Design Solutions to Improve the Antenna Bandwidth. This section provides an overview of the most commonly used techniques to increase the operating band of the printed monopole antennas. In particular, trapeziform and rounded ground planes, with notches near the feeding lines or with a zig-zag shaping of the upper edge, have proven to be particularly effective in increasing the operating band of said antennas.
A compact CPW-fed monopole antenna composed of an elliptical monopole and a coplanar trapeziform ground plane has been proposed in [35]. The antenna depicted in Figure 19(e) achieves a ratio impedance bandwidth of $21.6: 1$ and exhibits a nearly omnidirectional radiation pattern with a gain ranging between about 0.4 and $4 \mathrm{dBi}$. The radiating structure is a suitably modified version of the planar discone antenna. In particular, it is composed of an elliptical monopole fed by a tapered coplanar waveguide (CPW) and of a ground plane having the shape of a trapezoid to improve the energy emission. The antenna is realized on a dielectric substrate of thickness $1.524 \mathrm{~mm}$ having a relative dielectric permittivity 3.48. The antenna size is approximately $0.19 \lambda_{0} \times$ $0.16 \lambda_{0}$ at the lower working frequency. The antenna characteristics have been evaluated by using the full-wave FITbased CST Microwave Studio [25]. The antenna presents a simulated impedance band ranging from 0.40 up to $9.51 \mathrm{GHz}$ 


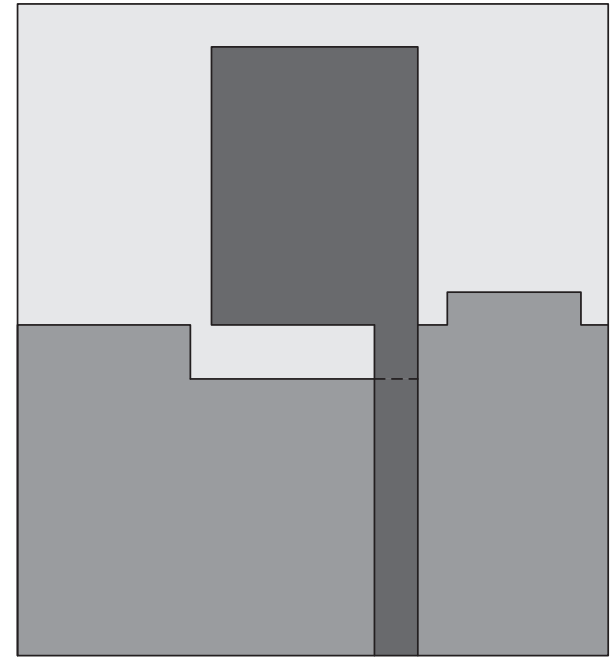

FIgURE 16: A microstrip-fed broadband circularly polarized monopole antenna with a stub integrated in the ground plane [36]. Dark gray upper metal face, medium gray bottom metal face, and light gray dielectric substrate.

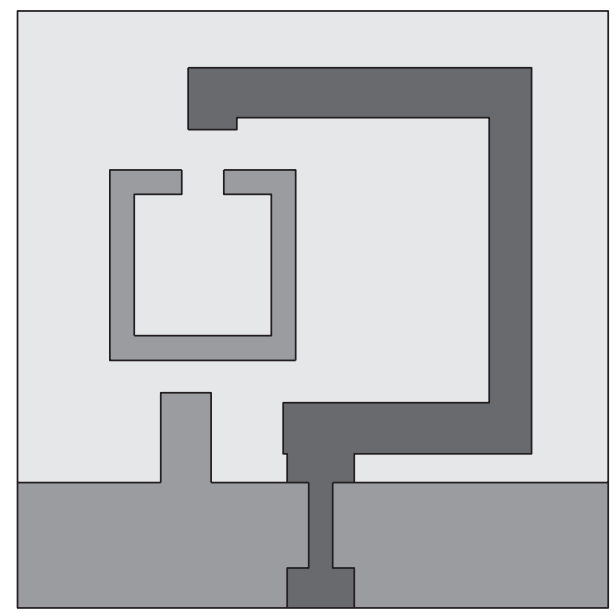

FIGURE 17: A C-shaped circularly polarized monopole antenna [38]. Dark gray upper metal face, medium gray bottom metal face, and light gray dielectric substrate.

and a measured operating band ranging from about 0.41 up to $8.86 \mathrm{GHz}$.

Best performances are obtained using an excitation technique making use of a trident-shaped tapered CPW to excite an elliptic monopole having a trapeziform ground plane [74] (see Figure 19(f)). The measured impedance bandwidth ranges from 1.0 to $24.1 \mathrm{GHz}$ useful for satellite navigation applications, wireless LANs, and ultrawideband systems.

The antenna can support many existing wireless services, including GPS (1.57-1.58 GHz), GSM1800 (1.71-1.88 GHz), PCS1900 (1.93-1.99 GHz), WLAN (2.5 or $5-6 \mathrm{GHz})$, and multiband GNNS and UWB (3.1-10.6 GHz). As for the metal plate monopole antenna ( $3 \mathrm{D}$ profile), the adoption of a fork feeding line improves the excitation of the vertical component of the current density with respect to the horizontal one

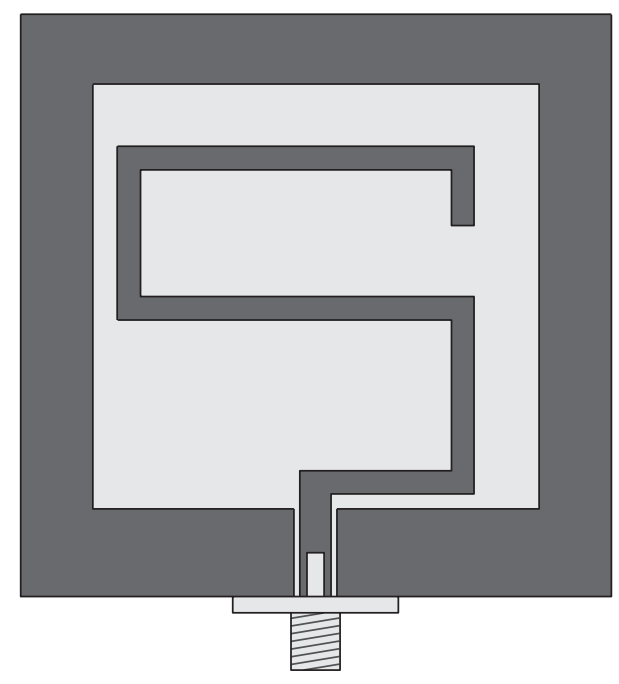

FIGURE 18: A dual-band circularly polarized rectangular slot antenna with an S-shaped monopole [85]. The excitation of edge currents together with those excited on the monopole is suitable to support a circular polarization. Dark gray upper metal face and light gray dielectric substrate.

on the monopole, making the antenna suitable to operate in a more extended frequency band. The antenna, printed on a dielectric substrate of thickness $1.524 \mathrm{~mm}$ and relative dielectric permittivity of 3.48 , has been characterized using the full-wave FIT-based CST Microwave Studio [25]. The radiation patterns show that in the $H$-plane the antenna has nearly omnidirectional radiation pattern at lower frequencies (from $1 \mathrm{GHz}$ to $5 \mathrm{GHz}$ ), while the $E$-plane radiation pattern shows a typical figure-of-eight at the frequency of $1 \mathrm{GHz}$, which is similar to a conventional dipole or to that of a biconical antenna. Further geometries of printed antennas based on trapeziform ground are shown in Figure 19.

An example of an antenna consisting of a dome-topped, bowl-shaped patch with a tapered truncated ground plane is shown in [72]. A notch is introduced below the feeding line near the patch for widening the antenna impedance bandwidth (see Figure 20). In this way the antenna is capable of operating in the frequency band $2.65-13 \mathrm{GHz}$ for UWB applications $(\mathrm{FBW}=132 \%)$. The antenna, printed on a FR4 substrate having $\varepsilon_{r}=4.4$, has dimensions of about $18 \mathrm{~mm} \times$ $20 \mathrm{~mm} \times 1.6 \mathrm{~mm}\left(0.47 \lambda_{0} \times 0.53 \lambda_{0} \times 0.04 \lambda_{0}\right.$, where $\lambda_{0}$ is the free-space wavelength at the central working frequency). From an analysis of the return loss, performed using the full-wave FEM-based HFSS [8], it appears that the antenna bandwidth typically depends on the width and depth of the notch realized in the ground plane and on the radius of the bottom part of the monopole. In particular, the upper frequency is greatly affected by the notch depth, while an optimum value of the gap between the radiator and ground plane promotes an increase in the electromagnetic coupling and therefore a widening of the operating band. Furthermore, the analysis of the current distribution excited on the metal surfaces reveals that the top of the radiator is little affected by the surface currents and therefore it has limited impact on the bandwidth. On the contrary, a higher current level 


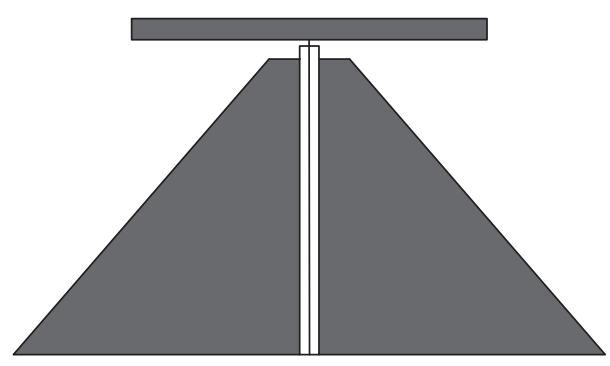

(a)

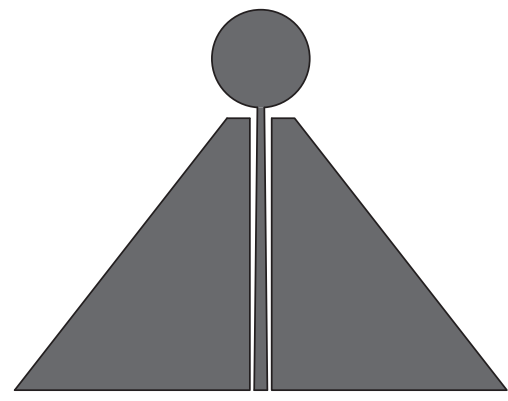

(d)

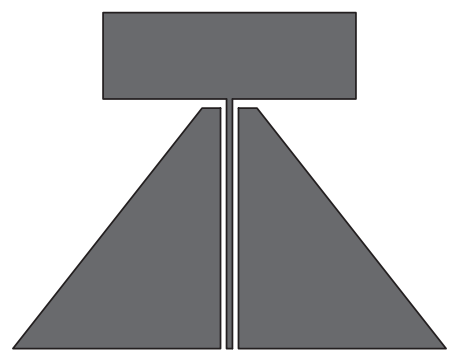

(b)

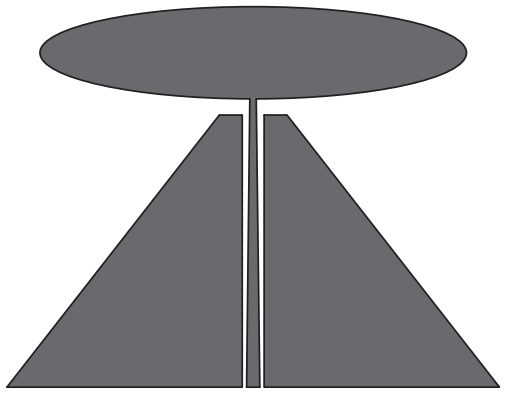

(e)

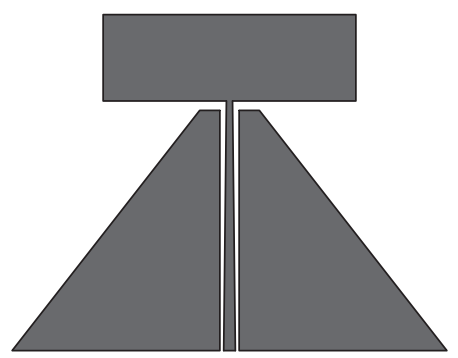

(c)

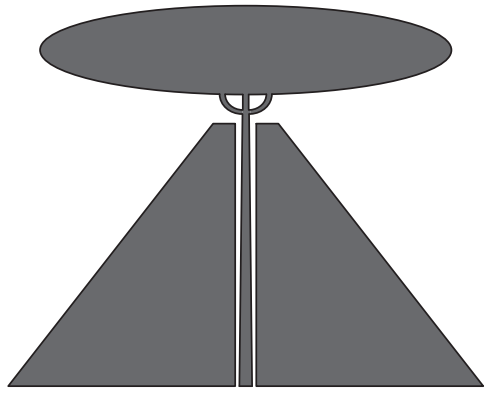

(f)

Figure 19: Printed antennas with trapeziform ground planes [74]. Different geometries of the monopole and of the excitation systems are shown in the figure.

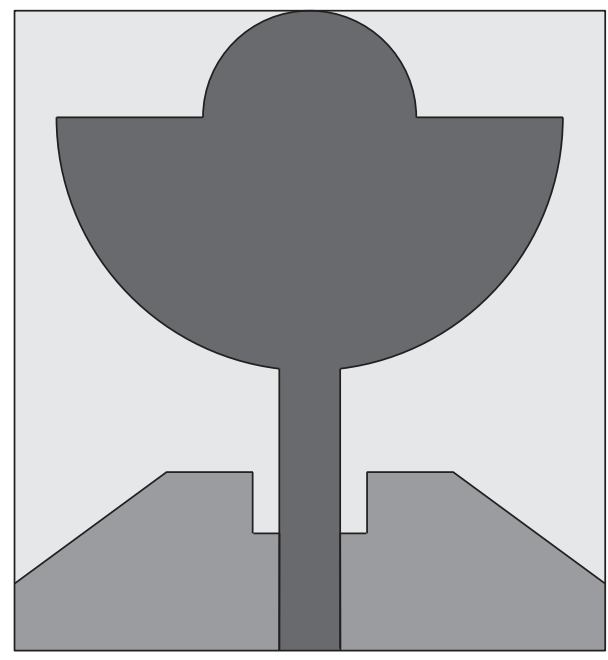

FIGURE 20: Miniaturized UWB printed monopole antenna with a dome-topped, bowl-shaped patch and truncated ground plane provided with a notch [72]. Dark gray upper metal face, medium gray bottom metal face, and light gray dielectric substrate.

is observed around the notch in the ground plane near the feeding line when the operating frequencies are lower, while in the lower patch region when the antenna working frequency increases.

Since antennas equipped with rounded ground plane have characteristics similar to those making use of trapeziform ground planes, for the sake of brevity they will not be taken into consideration. The reader interested in this subject can find information about this class of antennas in $[49,58,59]$.

An example of a rectangular monopole antenna, for wireless personal area network (WPAN), wireless body area network (WBAN), indoor localization, biomedical imaging, and UWB applications, making use of a zig-zag shaping of the upper edge of the ground plane (see Figure 21), is reported in [76]. The patch of the proposed antenna, having dimensions of $14.5 \mathrm{~mm} \times 14.75 \mathrm{~mm}$, is printed on the top side of a FR4 substrate of size of $30 \mathrm{~mm} \times 22 \mathrm{~mm}$, thickness $1.6 \mathrm{~mm}$, and relative dielectric permittivity of 4.6. The antenna band ranges from 3.8 up to $15.90 \mathrm{GHz}(\mathrm{FBW}=135 \%)$, while the maximum gain reaches $5.9 \mathrm{dBi}$. The increase of the operative band, introduced by the zig-zag profile of the upper edge of the ground plane, is due to the distributed modulation of the distance between the ground plane and the lower part of the monopole. Such modulation allows performing a more effective capacitive coupling between the ground plane and the monopole in a more extended frequency band, thus increasing the bandwidth of the original design by about $45 \%$ with respect to the solution without zig-zag contour. In addition, the sawtooth shaping allows reducing the overall size of the antenna and the levels of cross-polarization due to those currents excited in the ground plane by the presence of the asymmetric monopole [86].

Islam and Azim have shown [86] that the effect of the sawtooth shaping becomes more effective at high frequencies. The full-wave MoM-based IE3D [87] has been employed to analyze and design the antenna. With an average gain of $4.04 \mathrm{dBi}$, the antenna achieves a maximum gain of $5.9 \mathrm{dBi}$ at $9.4 \mathrm{GHz}$, while the gain variation is less than $2 \mathrm{dBi}$. 


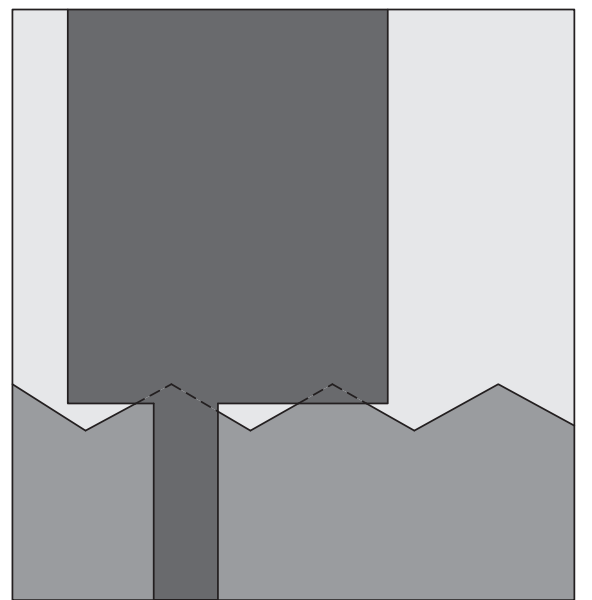

Figure 21: A monopole antenna making use of a zig-zag shaping of the upper edge of the ground plane [76]. The adopted zig-zag profile allows performing a more effective electromagnetic coupling between the feeding line and the monopole in a more extended frequency band. Dark gray upper metal face, medium gray bottom metal face, and light gray dielectric substrate.

A further radiating structure making use of a sawtooth ground plane, but with resonant elements placed under the rectangular monopole at the upper edge of the ground plane (where the magnetic field exhibits its maximum level) (see Figure 22) used to achieve three notched bands in the frequency ranges $3.26-3.71 \mathrm{GHz}, 5.15-5.37 \mathrm{GHz}$, and $5.78-$ $5.95 \mathrm{GHz}$, is presented in [77]. The antenna, which covers the frequency band ranging from 2 up to $11 \mathrm{GHz}$, has been proposed for UWB applications. A FR4 substrate, having $\varepsilon_{r}=$ $4.6, \tan \delta=0.02$, and thickness $1.6 \mathrm{~mm}$, has been employed to realize the antenna. The overall dimensions of the monopole antenna are of about $30 \mathrm{~mm} \times 22 \mathrm{~mm}$ corresponding to about $0.65 \lambda_{0} \times 0.48 \lambda_{0}$, where $\lambda_{0}$ is the free-space wavelength at the center frequency of the UWB band. In particular, an analysis of the current distributions excited on the metal filters [88] highlights an inductive behavior of the metal strips, whose entity depends on the length and width of the strips, while the distances between the filter arms or their gap regions determine a capacitive effect. The resonance frequency of the filter can be tuned by varying these two geometrical parameters. The full-wave MoM-based IE3D [87] has been employed to analyze and design the antenna. In conclusion, by measurements and simulations [77] it appears that the strips parameters and their positions with respect to the sawtooth edge influence the width and the center frequency of the notched bands.

4.4. Printed Monopoles with DGS. The removal of metal parts in the radiator and/or in the ground plane, in the neighborhood of which the reactive energy is mainly stored, can be used to increase the antenna impedance bandwidth. This procedure, known in the literature as defected ground structure (DGS), has led to the development of different geometries of slots and notches. The concept of DGS is basically inspired by photonic/electromagnetic bandgap structures (EBG/PBG) and metamaterials. In fact, EBG and PBG have the ability to inhibit the wave propagation in specific frequency ranges. On the other hand, metamaterials, whose dimensions of the intrusions should be lesser than a quarter wavelength, may not easily be engineered [78]. Given the lower structural complexity and the greater constructive simplicity, the DGS structures have found a greater spread in the design and realization of broadband antennas.

DGS technology is usually inserted beneath the microstrip feeding line since it usually acts as a capacitor and/or an inductance that compensates the original input impedance, thus widening the bandwidth. In fact, the presence of a defect in the ground plane modifies the surface current distributions with a remarkable impact on the antenna radiative performances [37]. Different efforts for improving the bandwidth [37], enhancing the gain [34], and reducing the size of printed monopole antennas [39] using DGSs are discussed in literature. In particular, they have been often employed in multiband and band-notched UWB systems to reduce the impact of electromagnetic interferences with other wireless systems $[62,79]$ as well as to suppress the higher order harmonics taking place in the active RF circuitry [89].

A large class of DGS structures (see Figure 23) together with some design formulas useful to identifying the working frequencies of said filtering structures is illustrated in [78]. These structures can also be employed in the feeding systems of printed antennas.

A trapezoidal monopole antenna, fed by a microstrip line printed on a dielectric substrate, and with two U-shaped slits, integrated in the ground plane (see Figure 24) to increase the antenna impedance bandwidth, has been presented in [37]. Using the mentioned DGS structure an antenna having a frequency band ranging between the 790 and $2060 \mathrm{MHz}$ $(\mathrm{FBW}=90.4 \%)$, which is higher of about $112.4 \%$ with respect to that without the DGS structure, is obtained. The antenna was analyzed and designed using the fullwave MoM-based IE3D [87]. The antenna, of dimensions of $60.4 \mathrm{~mm} \times 103.8 \mathrm{~mm}$, was realized on a $\mathrm{RO} 4003$ substrate with a dielectric constant of 3.38 and thickness of $1.524 \mathrm{~mm}$. A stub is placed near the transition between the microstrip line and the monopole to improve the impedance matching. Using two U-shaped DGS structures having different lengths, but identical width, two distinct bandgaps at 2.5 and $5 \mathrm{GHz}$ are obtained. Finally, the measurements results of the radiation patterns show a behavior similar to that of a conventional monopole antenna.

A further example of a broadband monopole antenna, fed by CPW and with cross-shaped DGS structures, to cover the bands $2.44-2.58 \mathrm{GHz}$ and $3.5-8.85 \mathrm{GHz}$ useful for WLAN applications $(2.4 / 5.2 / 5.8 \mathrm{GHz})$, WiMAX $(2.5 / 3.5 / 5.5 \mathrm{GHz})$, and point-to-point high-speed wireless applications (5.925$8.5 \mathrm{GHz}$ ), was proposed in [34]. The antenna is printed on a FR4 substrate, of dimensions $30 \mathrm{~mm} \times 28.8 \mathrm{~mm}$, with dielectric permittivity $\varepsilon_{r}=4.4$, loss tangent of 0.02 , and thickness $1.58 \mathrm{~mm}$ (see Figure 25). The antenna was analyzed using the full-wave FEM-based HFSS [8]. The measured gain varies between 4 and $10.8 \mathrm{dBi}$. 


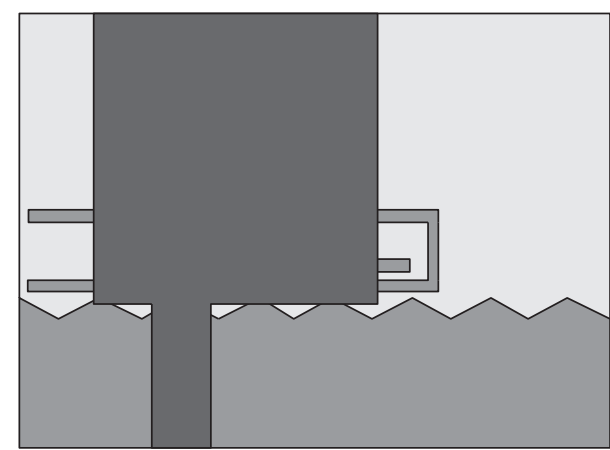

(a)

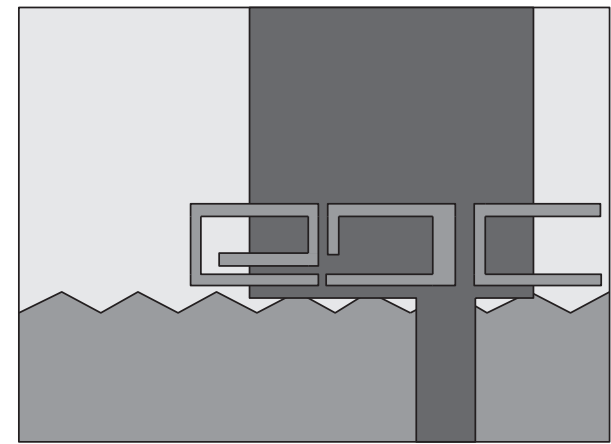

(b)

FIGURE 22: Monopole antenna printed on a sawtooth ground plane [77]. Resonant filters used to limit the electromagnetic emission in the frequency bands $3.26-3.71 \mathrm{GHz}, 5.15-5.37 \mathrm{GHz}$, and 5.78-5.95 GHz are shown in figure. Top view (a) and bottom view (b). Dark gray upper metal face, medium gray bottom metal face, and light gray dielectric substrate.

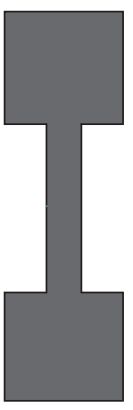

(a)

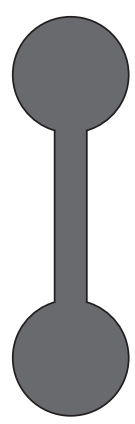

(b)

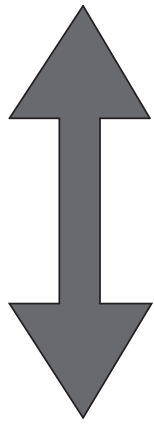

(c)

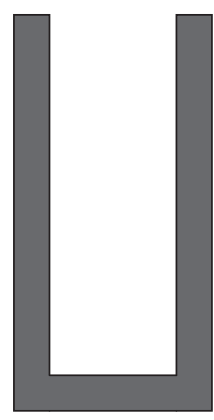

(d)

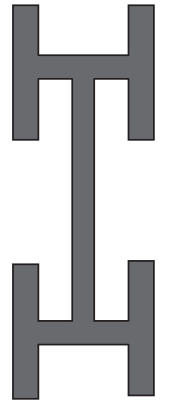

(e)

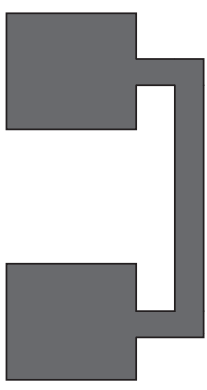

(f)

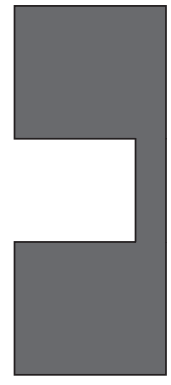

(g)

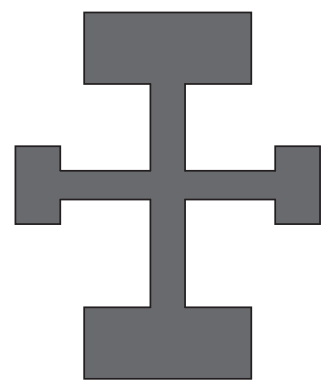

(h)

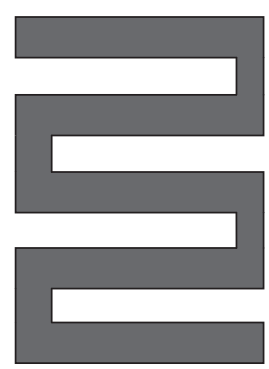

(i)

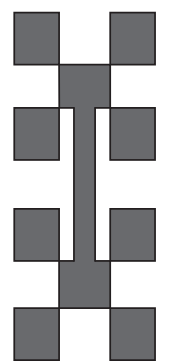

(j)

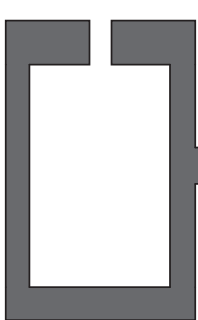

(k)

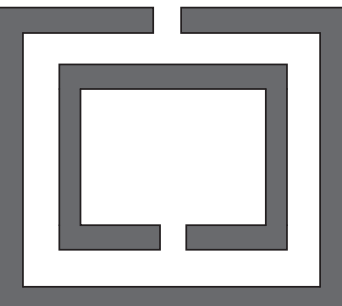

(q)

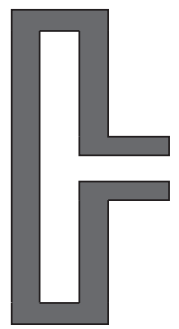

(l)

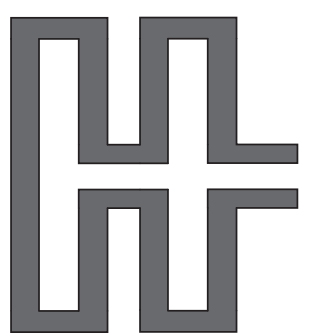

(m)

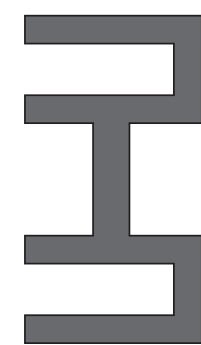

(n)

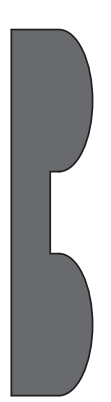

(s)

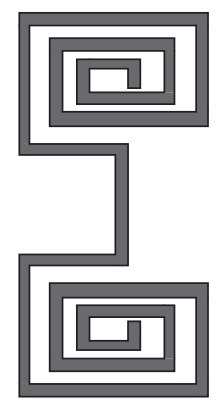

(t)

FIGURE 23: Some of the most common DGS structures employed in the applications [78]. (a) Dumbbell-shaped, (b) circular head dumbbell, (c) arrow head dumbbell, (d) U-shaped, (e) H-shaped, (f) squared heads connected with U-slots, (g) squared slots connected with narrow slot at edge, (h) cross-shaped, (i) interdigital, (j) fractal, (k) open loop dumbbell, (l) L-shaped, (m) meander lines, (n) U-head dumbbell, (o) double equilateral U, (p) V-shaped, (q) split-ring resonators, (r) concentric ring shaped, (s) half-circle, and (t) spiral-shaped. 


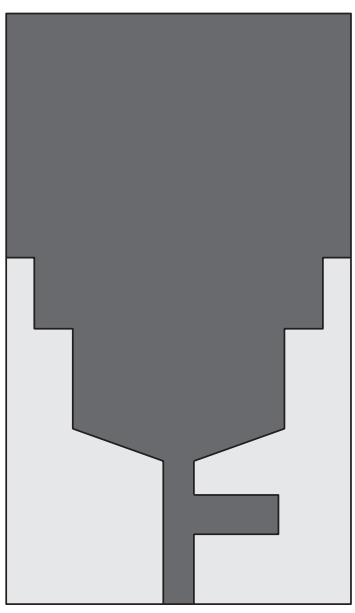

(a)

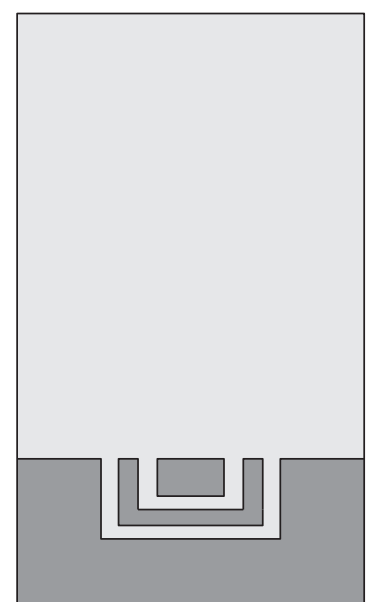

(b)

FIGURE 24: A microstrip monopole antenna with a defected ground structure [37]. The presence of the U-shaped DGS structure and of the stub is evident in the figure. Dark gray upper metal face, medium gray bottom metal face, and light gray dielectric substrate. Top view (a) and bottom view (b).

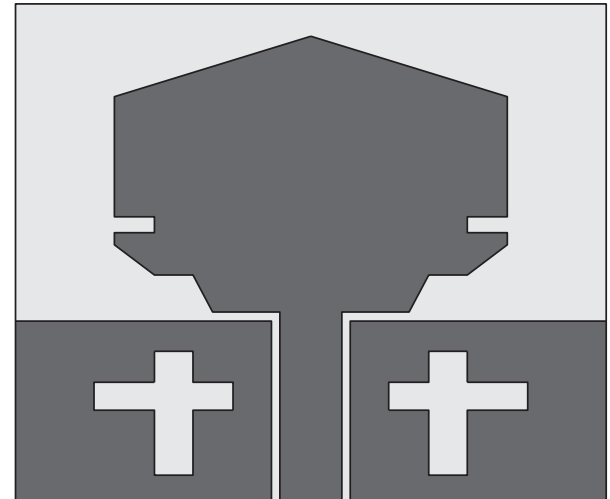

FIGURE 25: A CPW-fed monopole patch antenna with cross-shaped DGS [34]. The presence of the DGS structures is evident in the figure. Dark gray upper metal face and light gray dielectric substrate.

\section{Printed Slot Antennas}

Recently, printed slot antennas have been introduced because they are very attractive for wideband and UWB applications, especially the so-called printed wide-slot monopole antennas (see Figure 26(a)), which usually consist of a monopole (see Figure 26(b)) and a slot realized in a metal frame acting as ground plane (see Figure 26(c)). In fact, printed slot antennas typically exhibit wide impedance bandwidths and low near-field coupling which make them very suitable for the reduction of the EMC/EMI problems with the nearby devices and objects [90]. However, wideband performances and compact low-profile antennas are generally conflicting requirements that lead to a trade-off between the bandwidth and the antenna size. For this reason, many efforts have been done in literature for reducing the dimensions of slot antennas keeping the same wideband performances [90-92]. To this purpose, several slots geometries, such as square, rectangular, triangular, circular, and elliptical together with suitable excitation techniques making use of cross, fork, triangle, square, and arc monopoles, have been employed and investigated. In particular, a microstrip-fed antenna with a rectangular tuning stub and a tapered slot has been proposed in [91] (see Figure 26(a)).

The structure has a dimensions of about $22 \mathrm{~mm} \times 24 \mathrm{~mm} \times$ $1.6 \mathrm{~mm}\left(0.52 \lambda_{0} \times 0.57 \lambda_{0} \times 0.04 \lambda_{0}\right.$, where $\lambda_{0}$ is the free-space wavelength at the central frequency) and it is realized on a FR4 Roger substrate having dielectric constant of about 4.6 and loss tangent 0.02 . The obtained operational bandwidth, which ranges between 3 and $11.2 \mathrm{GHz}(\mathrm{FBW}=115.5 \%)$ covering the UWB applications, has been optimized by choosing adequate monopole and slot geometries. In particular, the rectangular monopole shows a better coupling with respect to rounded monopoles, while the tapered slot exhibits a larger impedance bandwidth than circular, elliptical, and square slots, as the current paths around the tapered slot edges are longer. Moreover, the gap between the monopole and the metal frame, illustrated in Figure 26(a), has been opportunely set in order to increase the coupling between the microstrip feeding line and the radiating slot. These characteristics have been carried out using the full-wave MoM-based IE3D [87]. Realized gain ranges between 5.4 and $9.8 \mathrm{dBi}$, while the radiation patterns at lower frequencies show low crosspolarization $(-20 \mathrm{~dB})$ levels. However, when the frequencies increase the polarization purity reduces. Finally, the antenna group delay variations are below $1.3 \mathrm{~ns}$, which can be retained acceptable in UWB communications systems.

A more compact printed wide-slot antenna has been proposed in [90]. In fact, the structure having dimensions of $16 \mathrm{~mm} \times 16 \mathrm{~mm}\left(0.41 \lambda_{0} \times 0.41 \lambda_{0}\right.$, where $\lambda_{0}$ is the free-space wavelength at the central frequency) is suitable to cover the 4.5-10.9 GHz frequency band (FBW $=83 \%$ ) for breast cancer detection and medical imaging. The antenna, formed by a pshaped slot excited by a rectangular monopole, is printed on a 


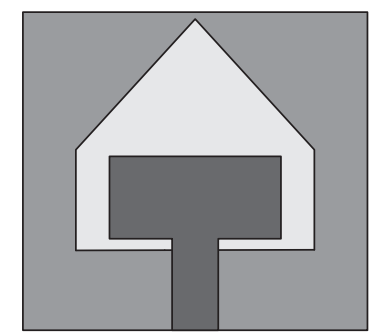

(a)
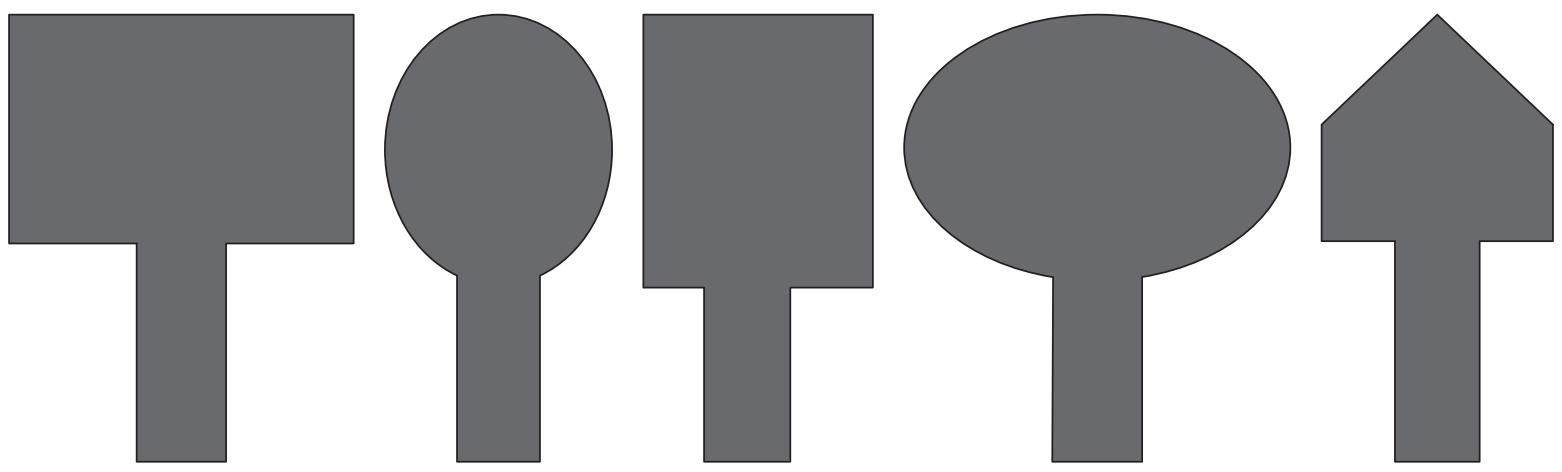

(b)
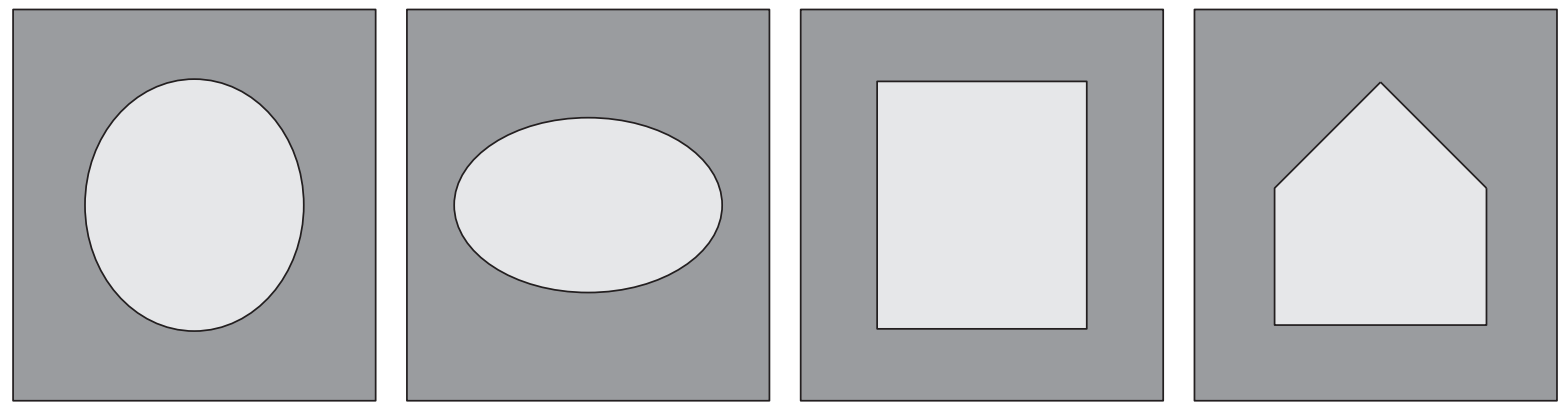

(c)

Figure 26: Example of a printed wide-slot monopole antenna (a) [91]. Monopole shapes (b) and frame geometries (c) adopted to realize printed wide-slot monopole antennas. Dark gray upper metal face, medium gray bottom metal face, and light gray dielectric substrate.

Rogers substrate of thickness $1.27 \mathrm{~mm}\left(0.03 \lambda_{0}\right)$ and dielectric permittivity of 10.2. A $50 \Omega$ microstrip feeding line connected to a SMA connector is used for the excitation of the antenna (see Figure 27).

The structure has been analyzed and designed with the full-wave FIT-based CST Microwave Studio [25]. Radiation patterns are typically donut-shaped and omnidirectional, resembling those of a dipole antenna. The antenna responses to UWB signals reveal a low distortion of the radiated field with values of the fidelity factor ranging within acceptable limits $(>0.5)$.

As the bandwidth of printed wide-slot antennas is mainly affected by slots and monopoles geometries, a deep investigation of binomial structures has been provided in [93]. In particular, six kinds of antennas have been simulated and measured for different orders of the binomial function $(N=$ $1,2,3,6,12$, and infinite). In particular, when $N=1$ the slot and the monopole have a triangular shape, while they

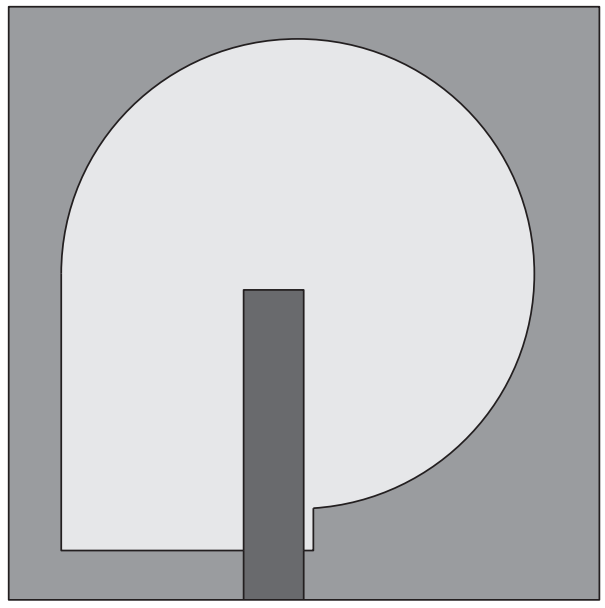

FIGURE 27: A compact UWB p-shaped wide-slot antenna for cancer detection [90]. Dark gray upper metal face, medium gray bottom metal face, and light gray dielectric substrate. 


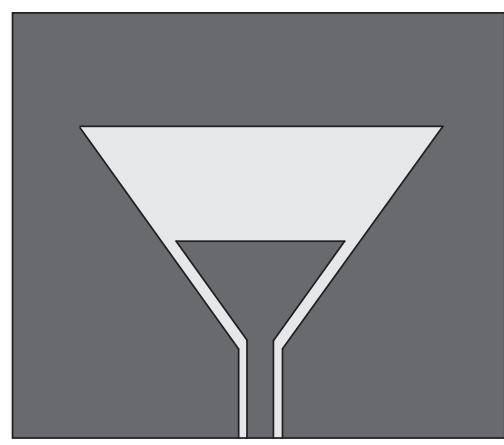

$N=1$

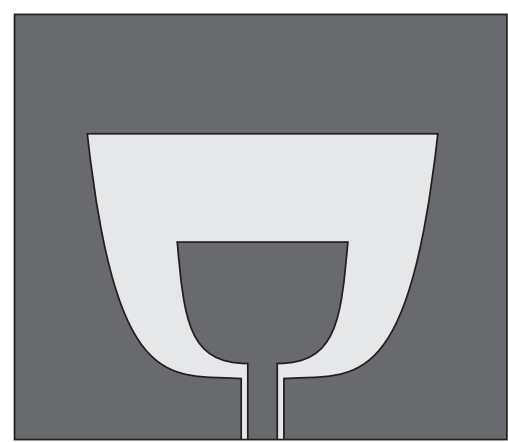

$N=6$

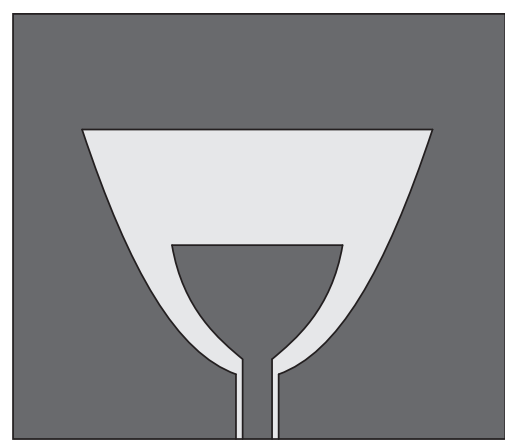

$N=2$

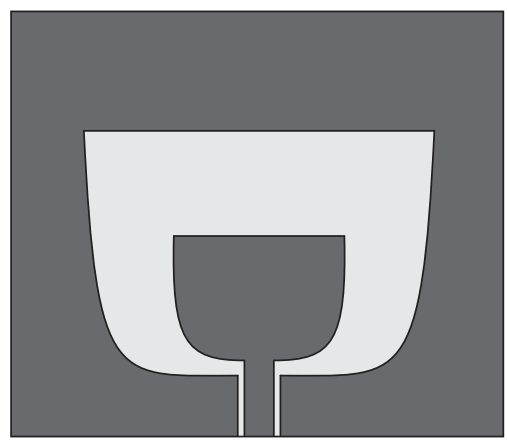

$N=12$

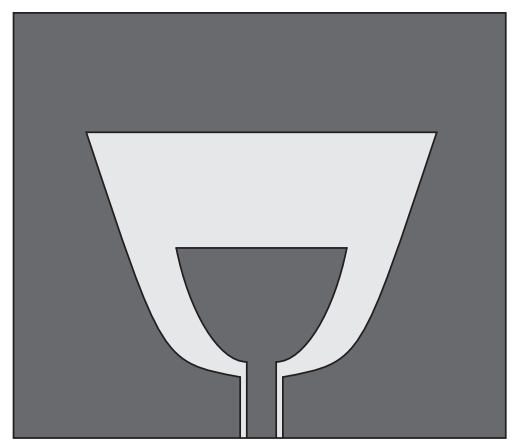

$N=3$

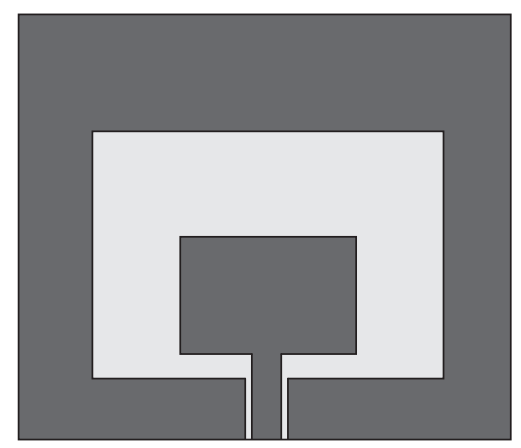

$N \rightarrow \infty$

FIGURE 28: The CPW-fed printed binomial wide-slot antennas [93]. Dark gray upper metal face and light gray dielectric substrate.

assume a rectangular shape when $N$ tends to infinite. Some examples of CPW-fed printed wide-slot antenna geometries for different values of the binomial order $N$ are depicted in Figure 28.

The proposed antennas are fabricated on a $35 \mathrm{~mm}$ $\times 40 \mathrm{~mm}$ dielectric Arlon TC600 substrate of thickness $0.787 \mathrm{~mm}$ and relative permittivity $\varepsilon_{r}=6.15$. The antenna features have been determined by means of the full-wave FEM-based HFSS [8]. Simulated fractional bandwidths range between $10.8 \%$ (triangular case) and $108.4 \%$ (rectangular case) as the antenna binomial order increases, while the measured values range between $9.3 \%$ and $99.7 \%$. In general, radiation patterns in the $E$-plane show cross-polarization levels that are lower than $-30 \mathrm{~dB}$ due to the symmetry of the structures. On the other hand, in $H$-plane antennas are asymmetric and thus cross-polarized components are higher. However, when $N$ increases the structures exhibit a reduced cross-polarized field level in the $H$-plane. Realized antenna gains vary between 3.3 and $5.5 \mathrm{dBi}$.

\section{Printed Dipole Antennas}

Although monopoles antennas present several advantages they do not radiate along their axes, making an array of monopoles not suitable to radiate in that direction. This can be disadvantageous if a radiating system, having a high-gain and compact dimensions, has to be integrated into a lowprofile host platform. To overcome these drawbacks printed

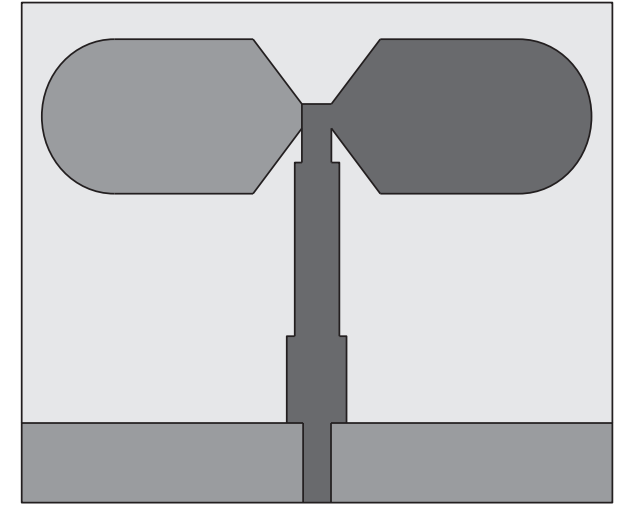

FIgURE 29: A printed double-sided rounded bow-tie antenna (DSRBA) [94]. Dark gray upper metal face, medium gray bottom metal face, and light gray dielectric substrate.

dipole antennas, having a proper geometry and equipped with a suitable metal screen, can be advantageously used. To this end, in this section a brief description of the main design solutions proposed in the literature is provided.

Two printed double-sided rounded bow-tie antennas (DSRBAs) for UWB applications (see Figure 29) have been proposed in [94]. The first antenna is printed on a dielectric substrate ROGER RO3600 of dimensions $36 \mathrm{~mm} \times 36 \mathrm{~mm} \times$ $1.27 \mathrm{~mm}$, dielectric permittivity $\varepsilon_{r}=6$, and loss tangent 0.002 . The second one is printed on a liquid crystal polymer 


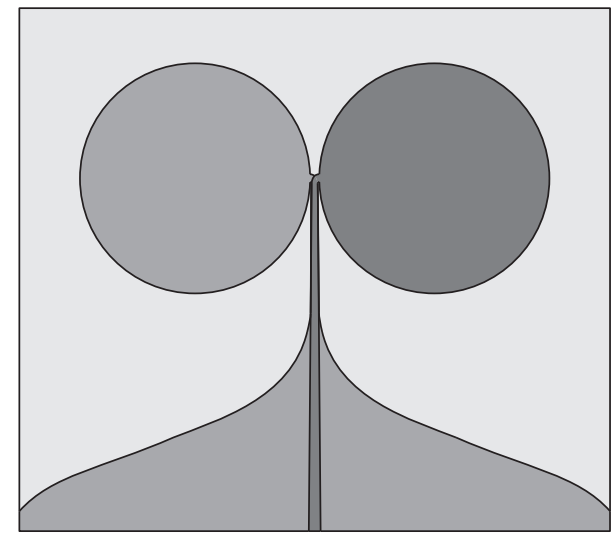

(a)

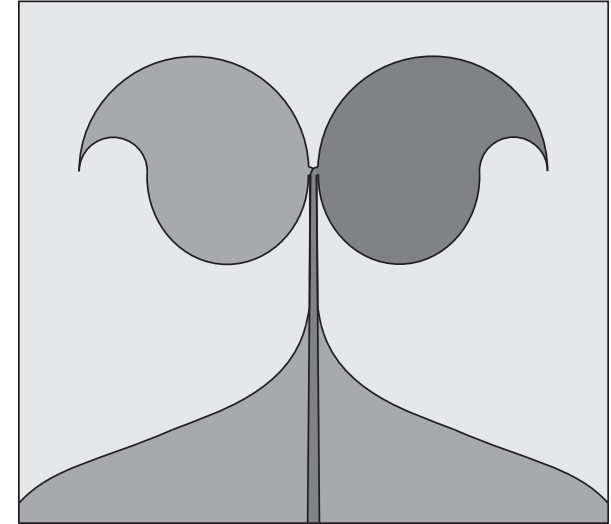

(b)

FIGURE 30: Geometry of the circular (a) and drop-shaped (b) antipodal dipole antenna employing an unbalanced feeding line [95]. Dark gray upper metal face, medium gray bottom metal face, and light gray dielectric substrate.

(LCP) of dimensions $32.4 \mathrm{~mm} \times 32.4 \mathrm{~mm} \times 1.14 \mathrm{~mm}, \varepsilon_{r}=$ 3.1, and $\tan \delta=0.002$, whose properties are suitable for conformal applications. Even though for both antennas the radiation characteristics are similar, it is shown that the geometry presenting rounded arms exhibits better performances in terms of bandwidth and cross-polarization levels with respect to the design solution based on flat-ended arms (rounded arms limit the level of the electric charges generally excited on sharp metallic edges, thereby reducing the reactive energy storage). In fact, a 59\% of additional bandwidth and $\mathrm{a}-10 \mathrm{~dB}$ lower cross-polarization level are achieved. The antenna gain ranges between 0.7 and $5.5 \mathrm{dBi}$, while the relative group delay is stable and acceptable within the UWB frequency spectrum. The main characteristics of the antennas have been derived using a homemade software based on a finite-element boundary integral formulation that employs tangential vector finite elements (TVFEs) and tetrahedral meshing and the full-wave FEM-based HFSS [8] typically used for high frequencies applications.

Two printed dipole antennas with antipodal circular and drop-shaped radiators have been proposed in [95] (see Figure 30). These antennas, which exhibit a lower level of surface waves and an excellent response to short pulse signals, are particularly useful for wideband, WLAN, WiMAX, and UWB applications. These antennas can be employed in portable devices (mobile PC, printers, scanners, DVD players, and digital projectors), in radio base stations, and in the nextgeneration textile fabrics for wearable applications, as they are printed on a CuClad217 thin flexible substrate that is more conformal and less prone to surface degradation. Such dielectric substrate has dimensions of about $51 \mathrm{~mm} \times 45 \mathrm{~mm} \times$ $0.38 \mathrm{~mm}$, dielectric permittivity $\varepsilon_{r}=2.17$, and $\tan \delta=$ 0.0009 . The antenna is excited by a suitable tapered transmission line to achieve an adequate $50 \Omega$ impedance matching within the UWB band. The two structures, which have been analyzed by means of a homemade locally conformal finitedifference time-domain (FDTD) numerical technique [96], are characterized by half-wavelength dipole-like radiation patterns up to $5.25 \mathrm{GHz}$. Furthermore, the particular contour of the drop-shaped antenna is suitable to guide the electromagnetic energy along the backside contour of the antenna arms toward the boresight direction resulting in a higher radiation level, as well as in a reduced energy emission in the backward direction, where electronic circuitry is typically located. This feature is also enhanced by the rear shaped planar reflector. The relative group delay ranges between -0.1 and $0.25 \mathrm{~ns}$ resulting in being excellent for UWB radar and wireless applications $[82,97]$. Finally, the antipodal characteristic of drop-shaped antenna allows obtaining compact antenna arrays for frequency or time-domain applications. These important features related to the particular radiative properties of said antenna make it particularly useful to be integrated not only in mobile devices, but also in high-gain RF base stations.

A different planar dipole antenna geometry has been introduced in [98]. The antenna, of overall dimensions $40 \mathrm{~mm} \times 65.3 \mathrm{~mm} \times 0.8 \mathrm{~mm}$, exhibits an operative bandwidth ranging between 2.8 and $10.9 \mathrm{GHz}(\mathrm{FBW}=118 \%)$ suitable for UWB applications. The dipole consists of two semielliptical-ended arms connected by a shorting bridge, which reduces the arms' length, increases the gain, enhances the response's quality, and improves the impedance bandwidth. The antenna is excited with a wideband balun composed of a Chebyshev three-section transformer connected to a $50 \Omega$ microstrip line. The feeding line and the impedance transformer are printed on a grounded dielectric slab having dielectric permittivity $\varepsilon_{r}=3.38$, while the dipole is printed in a region of the dielectric slab without ground plane to increase the antenna operating band (see Figure 31).

Numerical simulations have been performed by means of the full-wave MoM-based IE3D [87]. Although the antenna is relatively small, its peak gains are comparable with those of a bulkier dipole antenna and their values range between 2.6 and $5 \mathrm{dBi}$ with the balun and between 2.5 and $6.5 \mathrm{dBi}$ without the balun. It is observed that the three impedance steps realized in the balun are the major cause for spurious radiation and high cross-polarization levels. On the other hand, the shorting bridge avoids the current to be concentrated around 


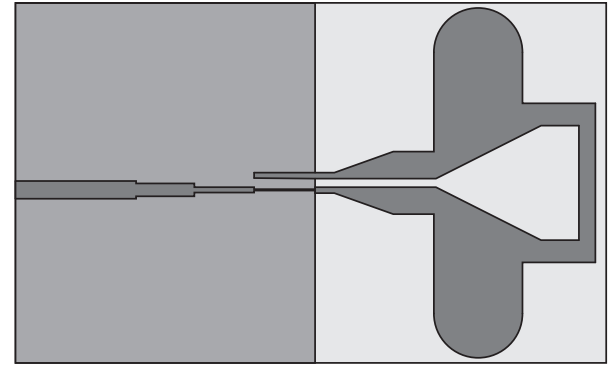

FIGURE 31: A UWB dipole antenna with semi-elliptical-ended arms and shorting bridge [98]. Dark gray upper metal face, medium gray bottom metal face, and light gray dielectric substrate.

the tapered V-shaped edges of the arms thus increasing the maximum gain of the antenna whose radiating process in this way mainly occurs along the shorting bridge. Finally, the transient antenna response to a sine modulated Gaussian monopulse shows that the antenna, provided with a shorting bridge, experiences a more stable linear phase response and a less spread of the received waveform with respect to the case without shorting bridge. Anyway, the UWB responses of both antennas are affected by small ringings.

A design solution useful to improve the radiation characteristics of an angle-dipole antenna for $5 \mathrm{G}$ mobile wireless communications, within the frequency band $26.7 \mathrm{GHz}$ and $30.6 \mathrm{GHz}(\mathrm{FBW}=13.6 \%)$, has been proposed in [99]. The printed dipole is realized on a dielectric substrate RT/Duroid 5880 having thickness of approximately $0.254 \mathrm{~mm}$, dielectric relative permittivity $\varepsilon_{r}=2.2$, and $\tan \delta=0.0009$. On each side of the substrate two $45^{\circ}$ angled-radiators, fed by a parallel-plate transmission line (see Figure 32), are printed. Anyway said dipole does not present high levels of frontto-back ratio, the gain is less than $3 \mathrm{~dB}$, and the radiation pattern is asymmetric in the H-plane. For this reason, the antenna is equipped with a resonant cavity of dimensions $5.2 \mathrm{~mm} \times 5.2 \mathrm{~mm} \times 3.5 \mathrm{~mm}\left(0.5 \lambda_{0} \times 0.5 \lambda_{0} \times 0.34 \lambda_{0}\right.$, where $\lambda_{0}$ is the free-space wavelength at $f=28 \mathrm{GHz}$ ) within which the radiator is located. In this way, the bandwidth is increased by $5.4 \%$, the gain is increased by about $4.5 \mathrm{~dB}$ reaching measured values ranging between 6.6 and $8 \mathrm{dBi}$, and the levels of frontto-back ratio are equal to approximately $18.5 \mathrm{~dB}$, while the beamwidth is approximately equal to $72^{\circ}$ for the $E$-plane and $69^{\circ}$ for the $H$-plane in such a way that the desired symmetry of the radiation pattern is restored. The resulting structure has been used to realize a linear array of eight elements excited via a $\mathrm{T}$-junction power divider in order to provide a tapered excitation. In this way, a maximum scan beam of about $40^{\circ}$, with side lobe levels (SLLs) of approximately $-15 \mathrm{~dB}$, is obtained. The full-wave FIT-based CST Microwave Studio [25] has been employed to analyze the antenna.

\section{Directional UWB Antennas}

Omnidirectional antennas useful for mobile applications are not suitable to support high-gain point-to-point wireless communications, as well as to realize spatial diversity schemes useful to reduce multipath fading effects, or high resolution UWB radar for objects detection and imaging applications. To this end, directional UWB antennas making use of suitable reflective structures have been recently proposed in literature [53-57, 73]. In this section some examples of this important class of antennas are illustrated.

A low-profile directional self-grounded bow-tie antenna for UWB applications has been presented in [73]. The antenna, which covers a frequency range of $2-15 \mathrm{GHz}$ with about $-10 \mathrm{~dB}$ reflection coefficient, stable radiation patterns, and good time-domain impulse response, can be usefully employed for sensor networks, UWB short-range communication systems, UWB radar for high resolution imaging systems, and super sensitive UWB radio astronomy. The antenna of dimensions $54 \mathrm{~mm} \times 58 \mathrm{~mm} \times 24 \mathrm{~mm}$, whose geometry is shown in Figure 33, assumes the so-called seagull-oversea configuration. The metallic arms are connected together, forming the metal reflector of the antenna, while a suitable balun, connected at the feeding point and housed behind the reflector, is employed to match a $50 \Omega$ microstrip feeding line to a $150 \Omega$ twin-line transmission line attached at the bow-tie arms. A careful full-wave analysis, performed using the FIT technique [25], has highlighted a directional antenna behavior with a directivity of about $5-8 \mathrm{dBi}$ and a maximum group delay of about $1 \mathrm{~ns}$, when the opening angle of the antenna arms is $60^{\circ}$. These radiation characteristics, induced by the full ground design solution, are strongly demanded in many applications, such as, UWB radar and tracking systems, UWB indoor geolocation systems, and UWB sensing and microwave tomography.

A UWB antenna with unidirectional radiation pattern, useful for broadband radar applications or for point-to-point wireless communications, consisting in a horizontal bowtie electric dipole, a center-fed loop antenna acting as a magnetic dipole, and a rectangular cavity, was proposed in [53]. The bow-tie electric dipole, printed on the top side of a dielectric substrate having relative permittivity 2.2 and thickness $0.508 \mathrm{~mm}$, is connected through six vias to two rectangular patches printed on the dielectric backside (see Figure 34). These patches are joined to the feeding line by means of a vertical balun consisting in a microstrip-tostripline transition having the purpose to match the $50 \Omega$ microstrip line to that of a $90 \Omega$ balanced stripline. This transition, mounted vertically on the center of the ground plane, is printed on a dielectric substrate with $\varepsilon_{r}=2.2$ and thickness $0.787 \mathrm{~mm}$. A series of high-impedance stub is introduced at the bottom of the balun to improve the impedance matching. Finally, two metal cylinders placed near the feed region connect the horizontal radiating structure to the ground plane, thereby allowing the realization of a center-fed loop antenna, which works as a magnetic dipole. The overall size of the structure including the reflector is $60 \mathrm{~mm} \times 50 \mathrm{~mm} \times 12.4 \mathrm{~mm}$. The full-wave FEM-based HFSS [7] was adopted to analyze and design the antenna. The impedance bandwidth ranges between 3.08 and $10.6 \mathrm{GHz}$ (FBW of about 110\%), while the realized antenna gain varies between 6.8 and $10.6 \mathrm{dBi}$. The radiation patterns show a good directional behavior characterized by a front-to-back ratio above $20 \mathrm{~dB}$ below $7 \mathrm{GHz}$, which drops to about $13 \mathrm{~dB}$ at 


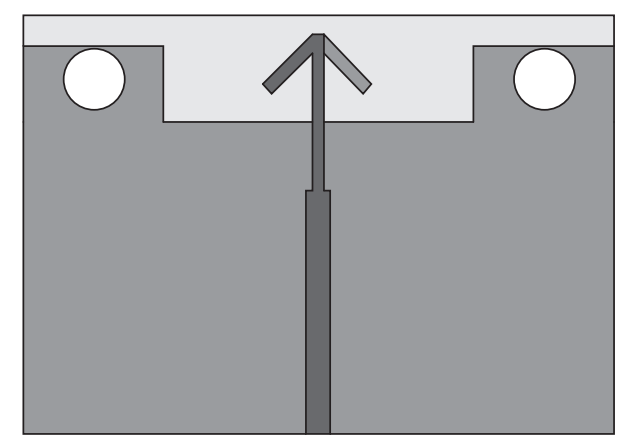

(a)

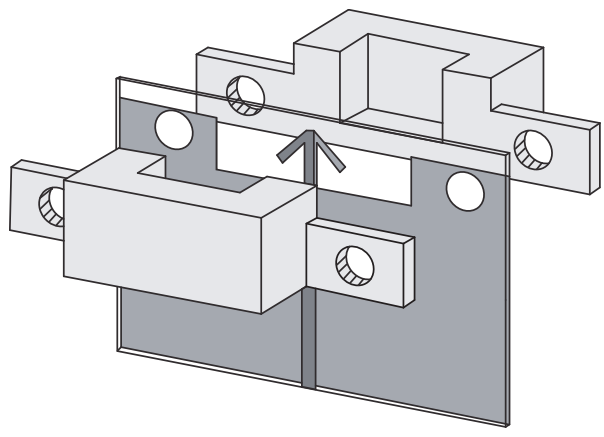

(b)

Figure 32: A cavity-backed angled-dipole antenna [99]. Front view (a) and tridimensional view (b). The flanged cavity is observable in the figure. Dark gray upper metal face, medium gray bottom metal face, and light gray dielectric substrate.

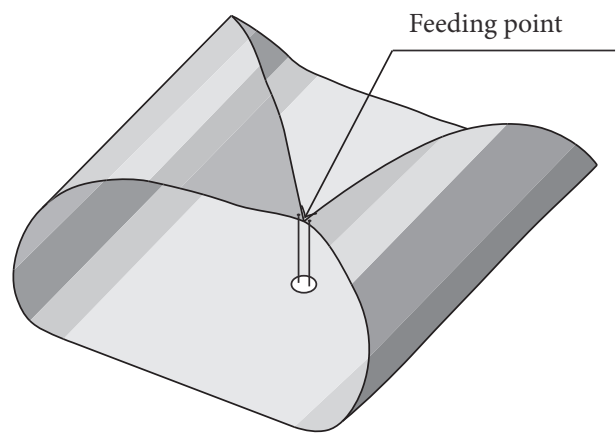

Figure 33: A low-profile directional self-grounded bow-tie antenna [73].

higher frequencies. The cross-polarization levels are below $-20 \mathrm{~dB}$ in the whole frequency band. The antenna response to UWB signals (coincident with 5th-derivative of the Gaussian pulse) shows a limited signal distortion and a ringing tail caused by the resonant processes taking place in the radiator and in cavity acting as reflector.

A UWB low-profile antenna for low UWB frequencies applications $(1-3 \mathrm{GHz})$ presenting directional characteristics and high front-to-back ratio is presented in [54]. These characteristics can be useful in ground-penetrating radar, microwave detection and imaging, and radio base stations. The antenna is realized using a slab of GIL GML 1032 substrate with relative dielectric permittivity 3.2 and thickness $1.52 \mathrm{~mm}$ on the opposite faces of which the arms of a bowtie-shaped flare are printed.

Two circular segments having a suitable radius are incorporated in each bow-tie arm to increase the effective edge length of the flare. In particular, in the upper face of the slab a $50 \Omega$ microstrip line, whose ground is composed of the bow-tie-shaped arm printed on the opposite face (see Figure 35), is adopted to excite the antenna. Both arms have two slits separated by a small gap. The terminations of the two arms are welded to two vertical metal plates welded in turn on a grounded dielectric slab having the same characteristics as the one used to realize the upper part of the antenna. The overall size of the proposed antenna is of about

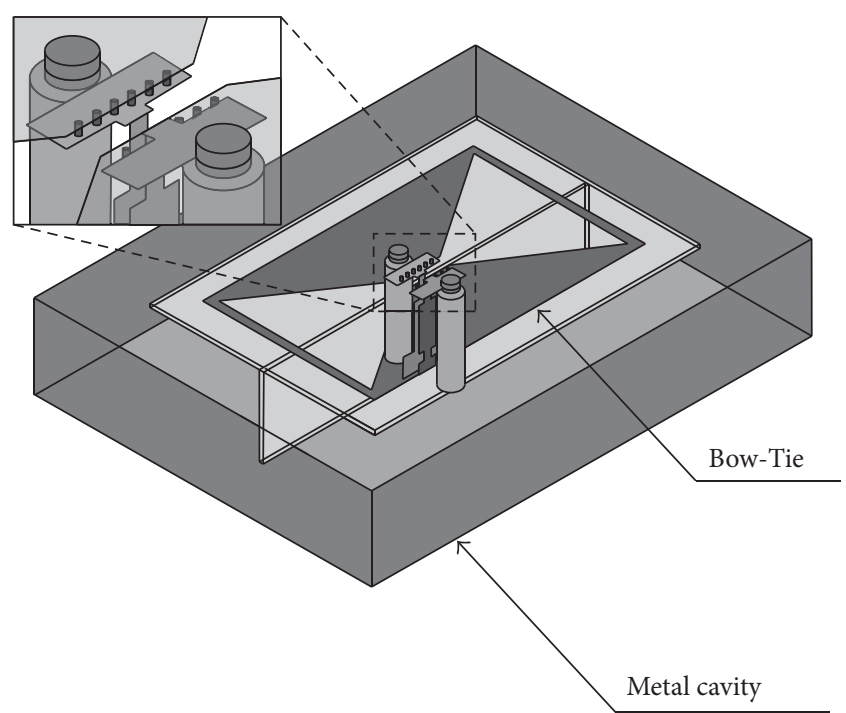

FIGURE 34: Geometry of the magnetoelectric dipole for unidirectional UWB communications [53]. The vias used to connect the antenna to the balun are indicated in the figure.

$70 \mathrm{~mm} \times 60 \mathrm{~mm} \times 15 \mathrm{~mm}$ corresponding to about $\lambda_{\text {eff }} / 3 \times$ $\lambda_{\text {eff }} / 4 \times 0.07 \lambda_{\text {eff }}$, where $\lambda_{\text {eff }}$ is the effective wavelength at the lower operative frequency. The antenna exhibits directional patterns with a fractional bandwidth of about $77 \%$ (from about 1.27 up to $2.57 \mathrm{GHz}$ ), average gain of about $5 \mathrm{dBi}$, up to $15 \mathrm{~dB}$ front-to-back ratio and $-20 \mathrm{~dB}$ of cross-polarization levels in both the $E$ - and $H$-plane. Moreover, thanks to the shorting walls placed perpendicular to the edges of the top and bottom layers, most of the side radiation is blocked. The antenna analysis and design have been performed using the full-wave FEM-based HFSS [8]. A good agreement between numerical results and measurements is observed.

A suitable choice of the antennas should be made when they are integrated in communication systems working in environments where the multipath fading can result in a significant reduction of the communication system performance. In such environments, antenna diversity techniques should be employed, since they can improve transmission 


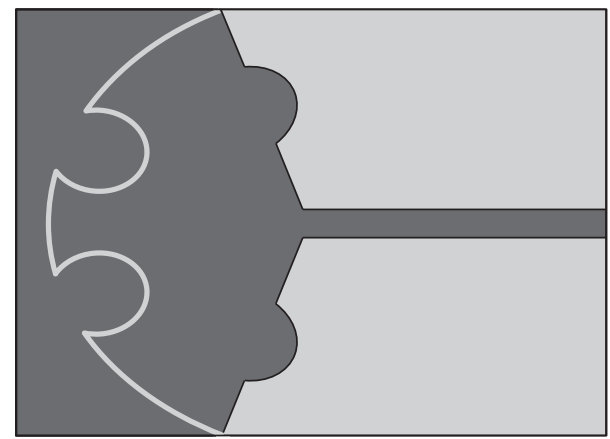

(a)

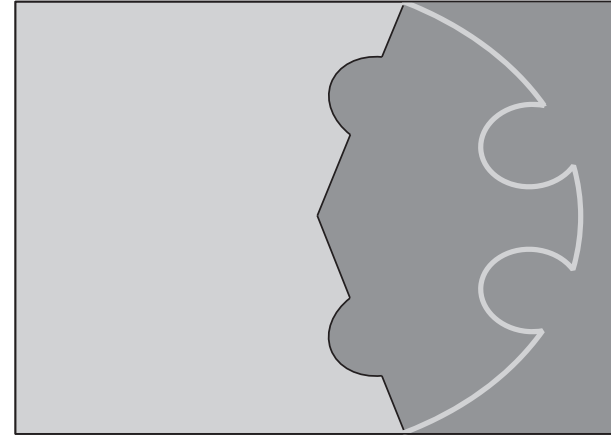

(b)

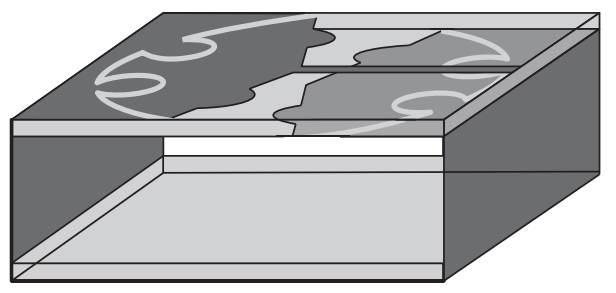

(c)

FIGURE 35: Geometry of a compact directional three-dimensional folded antenna for UWB applications [54]. Top view (a), bottom view (b), and tridimensional view (c).

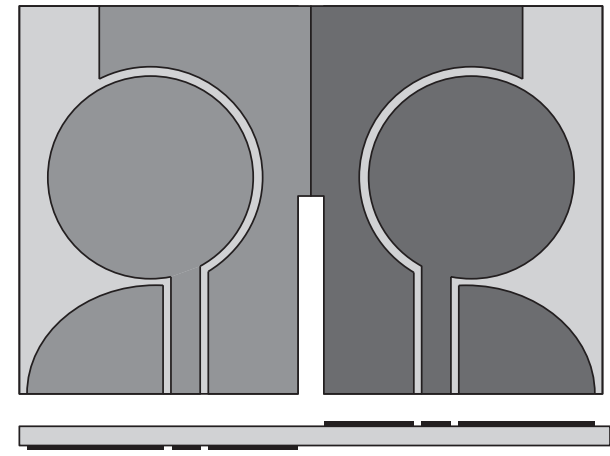

(a)

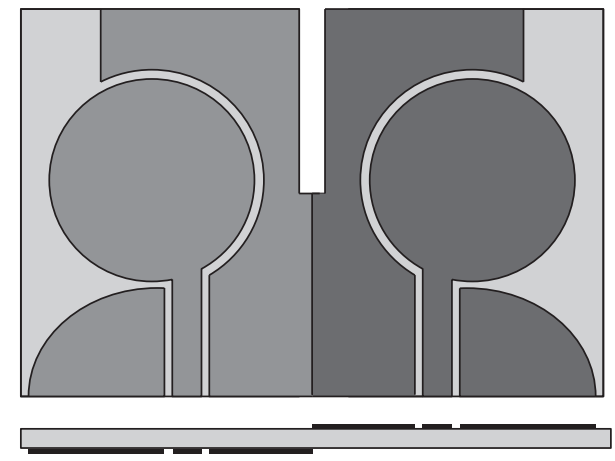

(b)

Figure 36: Geometry of the cross-configured directional UWB antenna [55]. The two structures (a) and (b) used to achieve a crossconfiguration antenna are shown in figure. The radiation process takes place in the region between the monopole and rounded ground plane.

quality by mitigating the multipath fading effects [18]. To this end, a UWB antenna which can be used as a directional antenna for mobile systems, or to realize multidirectional antenna systems, having high interelement isolation, for radio base stations, has been presented in [55]. The antenna is printed on a FR4 substrate of size $28 \mathrm{~mm} \times 44 \mathrm{~mm}$ (size $28 \mathrm{~mm} \times 88 \mathrm{~mm}$ for the four-element cross-configuration) having thickness $1.4 \mathrm{~mm}, \varepsilon_{r}=4.4$, and $\tan \delta=0.025$. The antenna characteristics have been carried out using the fullwave FIT-based CST Microwave Studio [25].

The radiator consists of a circular monopole, excited by a CPW line shifted with respect to the symmetry plane of the monopole, surrounded by a suitably shaped ground plane acting as reflector (see Figure 36). The reflector profile and its asymmetry with respect to the feeding line give rise in an antenna radiation beam oriented in the orthogonal direction (right/left) of the feeding line. In fact, the profiles of the monopole and the ground plane realize a sort of pseudoVivaldi antenna in the region where the ground plane is rounded. These features can be used to achieve a crossconfiguration composed of four monopoles. This configuration (see Figure 36) allows covering the four spatial quadrants maintaining a high interelement isolation $(>20 \mathrm{~dB})$ between the inputs of the four antennas allowing in this way achieving the required multidirectional pattern diversity characteristics. The experimental measurements performed on an antenna prototype have shown that the antenna gain varies between 4.3 and $7.5 \mathrm{dBi}$ in the frequency range $1.3-10 \mathrm{GHz}$. Therefore, in addition to UWB applications it can also be adopted for Bluetooth/Wi-Fi and WiMAX applications. 


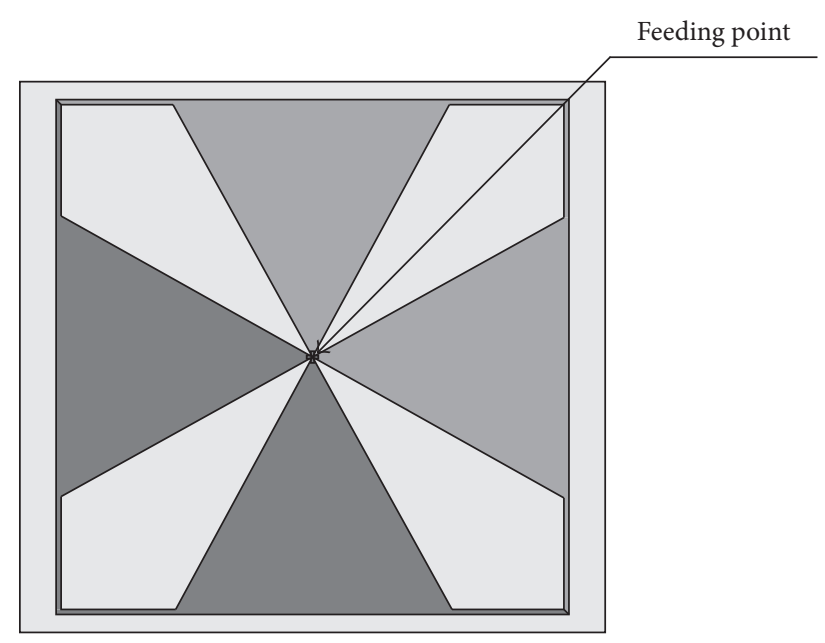

(a)

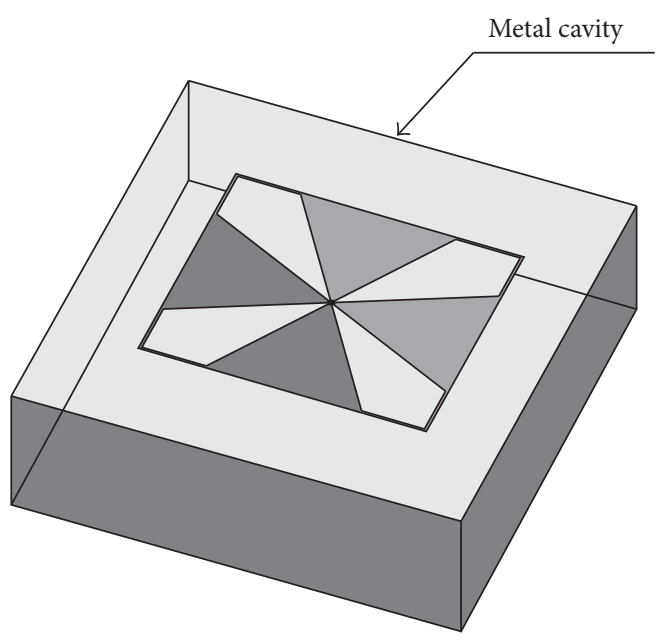

(b)

Figure 37: Geometry of a dual polarized wideband directional coupled sectorial loop antenna [56]. Top view (a) and tridimensional view (b).

Dual polarized wideband directional coupled sectorial loop antennas for radar and mobile base-station applications are presented in [56]. Two slightly different design solutions based on a dual polarized radiating element are proposed. For radar applications, the radiating element is used to excite the two degenerate modes $\left(\mathrm{TE}_{10}, \mathrm{TE}_{01}\right)$ of a cavity with square cross-section. The cavity of dimensions $160 \mathrm{~mm} \times$ $160 \mathrm{~mm} \times 60 \mathrm{~mm}$ is employed to improve the directivity useful for radar applications, such as those employed in through-wall imaging systems, since at least two antennas are typically used (one as transmitter and one as receiver). As for such applications higher VSWR can be tolerated, the antenna was designed so as to achieve the maximum bandwidth (approximately one octave) with a VSWR of 2.5. The proposed antenna structure, printed on a RO4003C substrate with relative permittivity 3.55 , loss tangent 0.0021 , and thickness $1.524 \mathrm{~mm}$, is shown in Figure 37. It consists of a dual polarized exciting element backed by a square cross-section cavity. The polarizing element consists of two orthogonal coupled sectorial loops antennas (OCSLAs). Each sectorial loop is composed of two opposite triangular patches and a rectangular ring around the outside. Two tapered microstrip lines arranged orthogonally and connected to two vertical coaxial cables placed inside the cavity are used to excite the antenna so as to support horizontally/vertically or circularly polarized radiated fields. Gains of about $10 \mathrm{~dB}$, with similar beamwidth in the $H$ - and $E$-plane, and high front-toback ratios are achieved.

A different design solution, useful for the mobile basestation applications (1710-2170 MHz), making use of the same radiating element placed over a ground plane, is instead adopted to obtain an antenna (size $71 \mathrm{~mm} \times 71 \mathrm{~mm} \times 35 \mathrm{~mm}$ ) with a wider beam, a higher operating bandwidth, and a better SWR (less than 1.5). This design solution employs a printed balun to excite the antenna. Without cavity a maximum gain of about $8 \mathrm{dBi}$ along the boresight is obtained. The analysis and design of both antennas have been performed using the full-wave FEM-based HFSS [8].

\section{Metamaterial Antennas}

Metamaterials are artificial structures composed of periodic or nonperiodic subwavelength macrocells, whose their effective medium properties can be controlled by designing the macrounits so as to realize special materials that do not exist in nature. Several structures, such as, the split-ring resonators (SRRs), the complementary SRRs (CSRRs), the transmission line- (TL-) based structures, and the artificial magnetic conductors (AMCs), have been introduced in literature with the aim of improving the performance of microwave circuits and antennas. In this section, some examples of this important class of antennas are provided with the aim to enable the reader to have a broader view of the technologies that can be used to realize wireless communication systems of new generation.

Two multiband antennas making use of metamaterials have been developed with the aim of covering the WiMAX 2.5/3.5/5.5 GHz and the WLAN 5.2/5.8 GHz bands [100]. Both the antennas are printed on a dielectric substrate with relative permittivity of 2.65 and thickness of $1 \mathrm{~mm}$. The overall dimensions of the radiating structures are of $35 \mathrm{~mm} \times 35 \mathrm{~mm} \times 1 \mathrm{~mm}$. The full-wave FEM-based HFSS [8] was adopted to analyze and design the antennas. The first antenna consists of a rectangular monopole loaded by means of an electric-LC (ELC) structure placed under the monopole. This structure transforms the monopole from a dual-band in a three-band antenna providing coverage in the bands $2.5 / 3.5 / 5.5 \mathrm{GHz}$, thereby meeting the requirements for the WLAN and WiMAX applications. Two slits arranged near the feeding line are used to improve the impedance matching. In addition to the ELC structure placed under the monopole, the second antenna integrates an electronic 


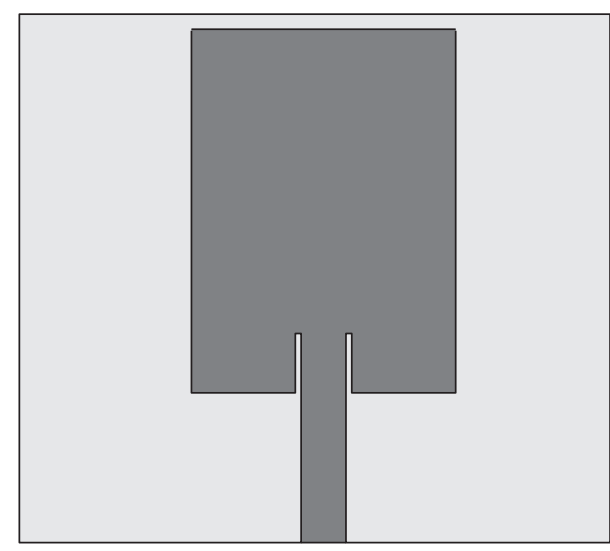

(a)

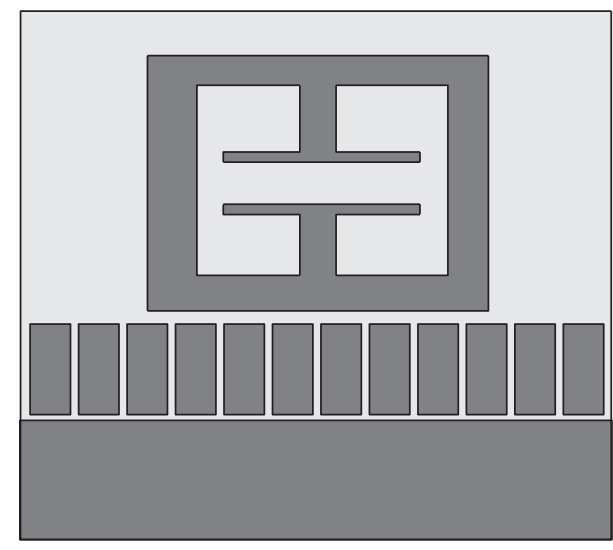

(b)

Figure 38: Geometry of a small metamaterial antenna with ELC structure [100]. Front view (a) and back view (b). Dark gray upper metal face and light gray dielectric substrate.

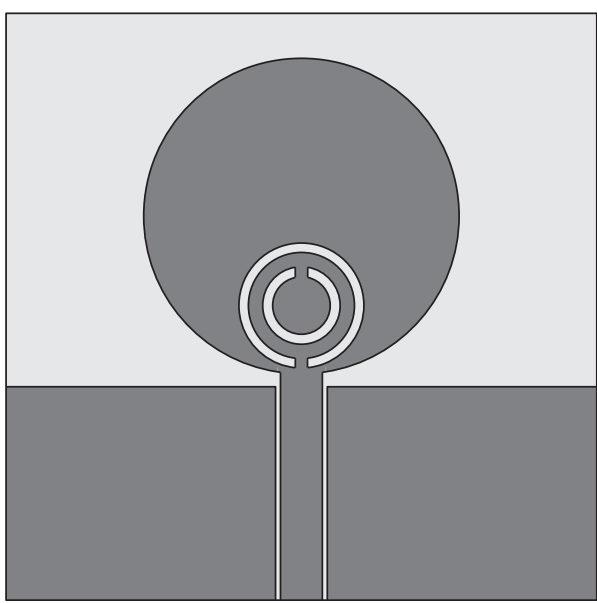

(a)

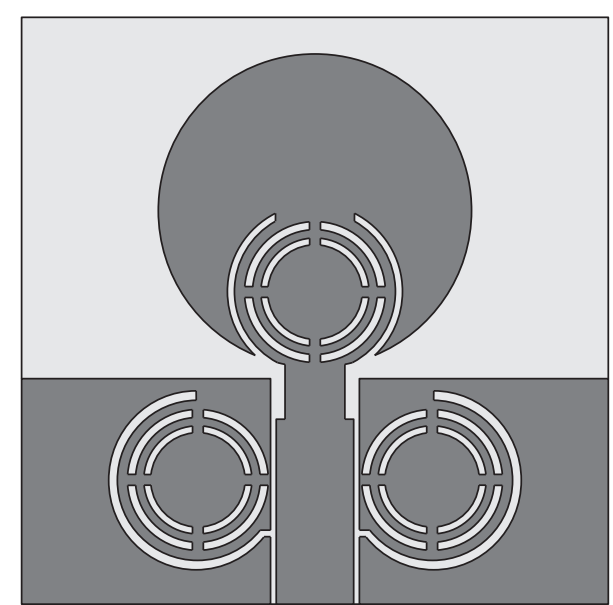

(b)

FIGURE 39: A UWB antenna employing metamaterials to realize a notched band useful to reduce interference with WLAN communication systems [101]. (a) CSRR integrated in the monopole; (b) CSRRs near the CPW. The CSRRs are located near the regions where the magnetic field exhibits its higher values. Dark gray upper metal face and light gray dielectric substrate.

band gap $(\mathrm{EBG})$ structure in the upper part of the ground plane (see Figure 38) to get a good impedance matching in frequency range $2.49-2.53 \mathrm{GHz}$ and a UWB behavior in the frequency range $2.95-6.07 \mathrm{GHz}$. It is observed that both antennas have stable omnidirectional radiation pattern in $\mathrm{H}$ plane and an 8-shaped radiation pattern in the $E$-plane.

A transparent antenna useful for short-range UWB applications making use of metamaterials was presented in [101]. This antenna, which can be easily attached on glass panels and windows of homes and buildings, is realized using a transparent conductive material known as silver-coated thin films (AgHT-4). This film, consisting of a thin layer of AgHT8 (height $0.053 \mathrm{~mm}$ ) placed on a PET polymer substrate of relative permittivity 3.23 , height $0.12 \mathrm{~mm}$, and surface resistivity $4 \Omega / \mathrm{m}^{2}$, has been employed to realize a UWB antenna of about $30 \mathrm{~mm} \times 30 \mathrm{~mm}$ (see Figure 39). To avoid interference with WLAN communication systems working in the same environment a bandstop filter tuned at $5.8 \mathrm{GHz}$, and making use of complementary split-ring resonators (CSRRs), has been integrated in the antenna. Split-ring resonators have been adopted as they are one of the artificial metamaterial structures that exhibit negative permittivity/permeability properties. In any case, three CSRRs structures, two in the ground plane near the CWP and one in the circular monopole, where the magnetic field exhibits its higher values (see Figure 39), are necessary to obtain a notched band of only $-4 \mathrm{~dB}$ at $5.8 \mathrm{GHz}$. Such poor performance occurs due to the high losses presented by the AgHT-4, which are responsible for a significant reduction of the resonant process taking place in the CSRRs. As expected the losses reduce the antenna gain that, within the UWB band, varies between $-11 \mathrm{dBi}$ and $-5 \mathrm{dBi}$. These results are similar with those observed 


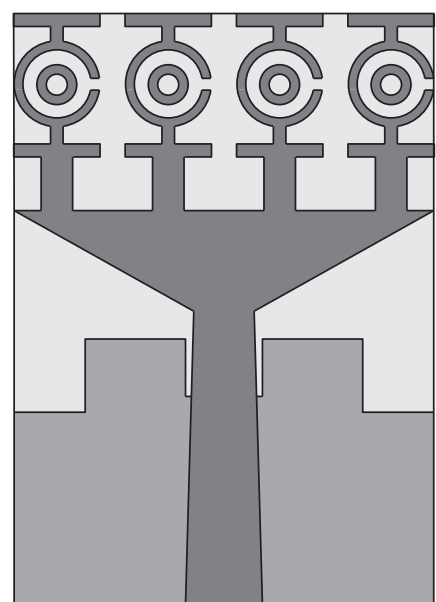

FIgURE 40: A metamaterial UWB antenna [102]. The four cells composed of two capacitance-loaded strips (CLSs), a split-ring, and a gapless ring, forming the metamaterial loading of the upper edge of the triangular patch, are evident in the figure. Dark gray upper metal face, medium gray bottom metal face, and light gray dielectric substrate.

in the transparent antennas. The analysis and design of the antenna have been performed using the full-wave FIT-based CST Microwave Studio [25].

A compact antenna based on metamaterial structures for UWB applications having a different geometry was presented in [102]. The antenna, whose overall dimensions are $27.16 \mathrm{~mm} \times 18.97 \mathrm{~mm}$, has been printed on both sides of a FR4 dielectric substrate of permittivity 4.6, loss tangent of 0.02 , and thickness $1.6 \mathrm{~mm}$. The antenna consists of four unit cells, which exhibit negative equivalent permeability and permittivity, attached to the upper edge of a triangular patch. Three rectangular slots are realized in the ground plane near the tapered microstrip feeding line (see Figure 40) to improve the impedance matching.

The metamaterial unit cell is composed of two capacitanceloaded strips (CLSs), a split-ring and a gapless ring. The four unit cells form a sort of periodic structure useful to provide an impedance bandwidth ranging from 3.07 up to $19.91 \mathrm{GHz}$ with excellent radiation characteristics, high-gain, efficiency, and adequate capability of pulse handling. The measured gain exhibits its maximum level of $8.57 \mathrm{dBi}$ at $13.5 \mathrm{GHz}$, a minimum level of $2.71 \mathrm{dBi}$ at $18.6 \mathrm{GHz}$, and an average level of $5.62 \mathrm{dBi}$ over the working frequency bands (3.0719.91 GHz). A quite stable omnidirectional radiation pattern in $H$-plane and an 8-shaped radiation pattern in the $E$-plane are observed. The gain variations and the ringing observed in the antenna response to pulse signals reveal the presence of resonant phenomena taking place in the structure forming the metamaterial as well as in the antenna geometry. The analysis and design of the antenna have been performed using the full-wave FIT-based CST Microwave Studio [25].

Additional information concerning the same subject can be found in [103-108].

\section{Wearable Antennas}

A growing interest in less bulky low-cost communication systems, which can be easily hidden into garments, helmets, glasses, or shoes, textile, and flexible wearable antennas is spreading among the scientific community. Indeed, wearable devices, exhibiting smaller profiles and lesser stiffness than the printed technological counterpart, can easily allow the civil defense, the police forces, the firefighters as well as the sportsmen, and so forth to focus directly on their specific activities. Moreover, the wearability of the communication system may be important also to support the remote control of biological parameters of not hospitalized patients or of athletes during sports activities. To this purpose, WBANs (Wireless Body Area Networks) for on-body, off-body, and in-body communications sceneries were regulated by the IEEE 801.15 standard [109].

Because of their relevance, in this section after an overview of the main dielectric and conductive textile materials useful to the realization of wearable antennas, some of the most recent progresses concerning this important class of antennas will be discussed.

9.1. Dielectric and Conductive Textile Materials for Wearable Antennas. In general, wearable antennas consist of metallic components (radiating patch and ground plane) printed on a dielectric substrate, whose geometries fall into the antenna classes analyzed in the previous sections. Therefore, for the sake of brevity the characteristics of the commonly employed materials for the realization of textile and flexible antennas are reported and analyzed in this subsection. In particular, some of their fundamental parameters such as conductivity, permittivity, and loss tangent are indicated for providing useful information during the design and realization phases of said antennas.

9.1.1. Dielectrics for Wearable Antennas. The dielectric materials used to realize wearable antennas are mostly affected by different factors such as frequency, temperature, surface roughness, and moisture content [110]. In particular, the level of the moisture absorbed by the fabrics is described by the Moisture Regain (MR), which is defined as follows:

$$
\mathrm{MR}=\frac{m_{\text {cond }}-m_{\mathrm{dry}}}{m_{\mathrm{dry}}},
$$

where $m_{\text {cond }}$ is the weight of the material at equilibrium after conditioning in a climate chamber and $m_{\text {dry }}$ is the mass of the material in dry state [110]. Some of the most common dielectric materials and their characteristics are reported in Table 2, while additional information of the mostly employed commercial fabrics is shortly described below.

Fleece is a common textile composed of synthetic woven polyester felted on both sides. As all the nonwoven synthetic materials that are classified as hydrophobic, fleece is a waterrepellent material [110]. However, it is not waterproof. Its lowloss tangent makes it very suitable for high-gain and highefficiency applications [125]. Fleece fabric is normally used for sportswear and leisurewear. 
TABLE 2: Nonconductive fabrics characteristics.

\begin{tabular}{|c|c|c|c|}
\hline Nonconductive fabric & $\varepsilon_{r}$ & $\tan \delta$ & Ref. \\
\hline Cordura $^{\circledR}$ & 1.90 & 0.0098 & {$[111,112]$} \\
\hline Cotton & 1.60 & 0.0400 & [110-113] \\
\hline $100 \%$ polyester & 1.90 & 0.0045 & {$[111,112]$} \\
\hline Quartzel ${ }^{\circledR}$ Fabric & 1.95 & 0.0004 & {$[111,112]$} \\
\hline Cordura/Lycra $^{\circledR}$ & 1.50 & 0.0093 & {$[111,112]$} \\
\hline Jeans cotton & 1.67 & - & {$[114]$} \\
\hline Polycot & 1.56 & - & {$[114]$} \\
\hline Polyester & 1.44 & - & [114] \\
\hline Foam (polyurethane) & 1.24 & 0.0213 & [110] \\
\hline Rubber protective foam & 1.495 & 0.016 & {$[110,115]$} \\
\hline Polyethylenteraphthalate (PET) & 1.173 & 0.0027 & [110] \\
\hline Pile & 1.12 & 0.009 & [116] \\
\hline Denim & $1.6-1.65$ & 0.05 & [117] \\
\hline Polydimethylsiloxane (PDMS) & From 3 to 20 & $<0.02$ & {$[118,119]$} \\
\hline Velcro $^{\circledR}$ & 1.34 & 0.006 & [120] \\
\hline Leather & 2.72 & 0.02 & [121] \\
\hline Silk & 1.75 & 0.012 & {$[122]$} \\
\hline Felt & $1.215-1.225$ & 0.016 & [117] \\
\hline Fleece & 1.17 & 0.0035 & {$[122,123]$} \\
\hline Flannel $(0.3-20 \mathrm{GHz})$ & 1.7 & 0.0250 & [124] \\
\hline
\end{tabular}

Denim fabric is a typical textile substrate with firm surface, which is robust against compression operations. Besides, denim features durability, inelasticity (useful for keeping constant the dielectric permittivity under bending conditions [122]), cost-effectiveness, and low thickness (about $0.7 \mathrm{~mm}$ ) [126]. However, when under wet conditions or after fully immersion in water, a degradation of UWB signals can be observed [127].

Felt is often used for textile antennas as it is flexible, soft, and comfortable. Antennas employing felt substrates exhibit lower return loss values with respect to those using fleece, foam, and conventional FR- 4 substrates. In addition, monopole antennas realized with the fleece substrate show the wider fractional impedance bandwidth [128].

Cotton is an easy-care textile, strong, durable, and more cost-effective than other substrates such as denim, felt, and jeans [129]. However, cotton is more suitable than other rigid substrates when bending and crumpling conditions occur.

9.1.2. Conductive Textiles. To integrate metallic parts in a textile flexible patch antenna, some conductive fabrics, whose characteristics are reported in Table 3, have been analyzed for different kind of applications and under several environmental conditions. Among them, four kinds of conductive materials can be identified:

(i) Flectron ${ }^{\circledR}$

(ii) Shieldex $\operatorname{Nora}^{\circledR}$

(iii) Shieldit Super ${ }^{\circledR}$

(iv) Shieldit Zelt ${ }^{\circledR}$
TABle 3: Conductive textiles characteristics.

\begin{tabular}{lccc}
\hline $\begin{array}{l}\text { Conductive } \\
\text { material }\end{array}$ & $\begin{array}{c}\text { Surface } \\
\text { resistance } \\
(\Omega / \text { square })\end{array}$ & $\begin{array}{c}\text { Equivalent } \\
\text { conductivity } \\
(\mathrm{S} / \mathrm{m})\end{array}$ & Thickness $(\mathrm{mm})$ \\
\hline Flectron [130] & 0.07 & $0.79 \times 10^{5}$ & 0.18 \\
Shieldit Super [131] & 0.05 & $1.18 \times 10^{5}$ & 0.17 \\
Shieldex Nora [132] & 0.03 & $3.33 \times 10^{5}$ & 0.1 \\
Zelt [133] & 0.01 & $16.7 \times 10^{5}$ & 0.06 \\
$\begin{array}{l}\text { Copper foil [131] } \\
\text { Pure copper taffeta }\end{array}$ & 0.0005 & $571 \times 10^{5}$ & 0.035 \\
fabric [131] & 0.05 & $2.50 \times 10^{5}$ & 0.08 \\
\hline
\end{tabular}

Flectron Nickel/Copper Polyester Nonwoven is a unique fabric, manufactured using a patented, proprietary technology. The base layer is copper, while the outer layer, made of nickel, is robust against corrosion. This technology combines the properties of these metals with the lightweight, permeability breathability, and flexibility of a nonwoven textile. Nickel/ Copper Polyester Nonwoven textile offers excellent surface conductivity $(<0.07 \Omega$ /square), shielding effectiveness, soldering robustness, and corrosion resistance for a variety of applications (architectural shielding, gaskets, shielding materials, and ribbon) [134]. Some other kinds of Flectron textiles with different characteristics can be found in [135].

Nylon fabric, Shieldex Nora from Shieldex Trading, Inc., is a woven high conductivity metal (surface resistivity of $0.03 \Omega$ /square) which is suitable for RF circuits and antennas operating in the ISM (Industrial Scientific Medical) band. It is a nylon fabric, plated with three metallized layers $(\mathrm{Ni} / \mathrm{Cu} / \mathrm{Ag})$, 
which ensure protection against corrosion and abrasion, as well as extreme flexibility [132].

Shieldit Super is a kind of material produced by Less EMF Inc., useful for pouches, wall covering, gaskets, grounding, and liners. It is a high quality flame retardant fabric for radiofrequency and microwave shielding. It is plated with nickel and copper (for achieving excellent shielding and robustness against corrosion), and it is coated on one side with a nonconductive hot melt adhesive (which is activated at $130^{\circ} \mathrm{C}$ ) so that it can be ironed on cotton, wood, glass, and paper or rolled into a tube and heat seal. It exhibits typical thickness of about $0.17 \mathrm{~mm}$ with surface resistivity below $0.05 \Omega$ /square. It can be resistant to maximum temperatures of $200^{\circ} \mathrm{C}$ and it can be easily washed $[124,136]$.

Shieldit Zelt is a high conductivity nylon fabric (the surface resistivity is lower than $1 \Omega$ /square) coated with copper and tin. It is an excellent material for shielding and it can be easily cut and sewn offering excellent corrosion resistance. It can be used for curtains, bedding, garments, wire sheathing, enclosures, and so forth [133, 137]. Some vendors that sell conductive fabrics are Less EMF Inc., Swift Textile Metallizing, LLC, Marktek Inc., Shieldex, and Metal Textiles Corporation [138].

9.1.3. Embroidered Textiles. The so-called e-textiles are usually made of conductive yarns embroidered in warp and weft directions, typically plated on a polymer-based substrate. In some cases, conventional and conductive threads are used together to reduce the cost of the textile antenna, which usually is very expansive. Nevertheless, the e-textile antenna typically achieves lower gain values with respect to a conventional antenna made of an all-metallic radiator as it has been highlighted in [139]. In fact, the weft in the ground plane can induce a local anisotropic behavior or abrupt changes in the current paths. Therefore, soldering and conductive epoxy have been used to enable orthogonal current paths so as to achieve acceptable gain performances [139].

9.1.4. Conductive Inks. Textile and wearable antennas sometimes need to be washed and cleaned. To this purpose, in [140] the authors investigated the differences between conductive inks and electrotextiles behaviors during several wet-drywet cycles. In particular, the proposed printed antennas realized by means of conductive inks have been covered with a thermoplastic polyurethane coating with the aim to protect the structures from water and corrosion. After performing several washing cycles, it was observed that the combination of an external coating and a conductive ink for the antenna realization provides a more stable antenna behavior maintaining a high radiation efficiency, less fluctuations of the reflection coefficient, and a more restrained resonant frequency shift. In [140], the conductive ink was printed on the antenna substrate by means of a screenprinting technique, using a semiautomatic Johannes Zimmer Klagenfurt-type Mini MDF 482 machine. In [141], conductive inks employed in flexible, lightweight, thin textile antennas were investigated for life-vest and maritime emergency applications. The conductive materials have been characterized

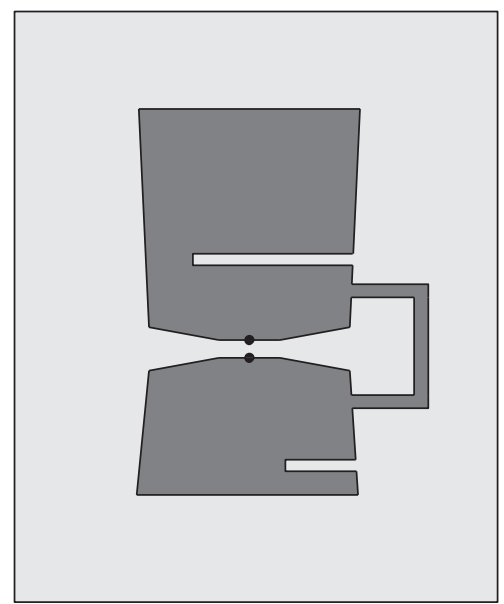

FIgURE 41: A triple band e-textile antenna [143]. The black dots indicate the feeding points. Dark gray upper conductive polymer fibers (e-fibers) and light gray dielectric textile substrate.

under several environmental conditions such as salt fog, salt water, humidity, and corrosion. A special attention has been given to silver-based inks. From the investigations carried out by the authors [141] it was observed that antennas keep stable behaviors and high efficiencies only if coated with at least two-sided laminates and two printed layers, especially in presence of salt fog. Dielectric substrate on which the antenna has been printed is a lightweight and low-loss foam featuring low moisture-absorption rate.

In Table 4 some conductive inks for wearable antennas are reported.

9.2. All-Textile Wearable Antennas. In [143], an e-fiber was used as a conductive patch of dimensions $60 \mathrm{~mm} \times 100 \mathrm{~mm}$ opportunely sewed to a polymer-based substrate (PDMS: $\left.\varepsilon_{r} \simeq 3, \tan \delta<0.001\right)$ to realize a triple band e-textile antenna (see Figure 41) covering the GSM, PCS, and WLAN $(2.4 \mathrm{GHz})$. The loaded loop and the narrow slots realized on the two patches are critical for achieving a good impedance matching at all three frequency bands. As the 332-strand efibers textile has a high conductivity and resistivity of only $0.8 \Omega / \mathrm{m}$, the e-textile antenna shows performances similar to its all-metallic counterpart, exhibiting $2 \mathrm{dBi}$ of realized gain. In particular, higher radiative performances are observed when the antenna is located on the front or on the back of the human body. Finally, it should be noticed that the antenna maintains its performance even after repeated flexing and washing/drying cycles.

An all-textile antenna with full ground plane (ATAFGP) useful for UWB and WBAN communications has been presented in [144]. The antenna, whose geometry is depicted in Figure 42, employs a full ground plane to achieve high radiative performances and excellent electromagnetic isolation with the users. The analysis and design of the antenna have been performed using the full-wave FITbased CST Microwave Studio [25]. The antenna is realized using Shieldit Super as conductor and felt as dielectric substrate. The Shieldit Super from Less EMF Inc. (Latham, 
TABLE 4: Conductive inks characteristics.

\begin{tabular}{|c|c|c|c|c|}
\hline Conductive ink & Resistivity $(\mu \Omega-\mathrm{cm})$ & Sintering temperature $\left({ }^{\circ} \mathrm{C}\right)$ & Viscosity $(\mathrm{mPa} \cdot \mathrm{s})$ & Fabrication technique \\
\hline $\begin{array}{l}\text { NPS-J (silver) } \\
{[141]}\end{array}$ & 3 & $210-220$ & 11 & Inkjet printing \\
\hline $\begin{array}{l}\text { Cabot CCI-300 } \\
\text { (silver nanoparticles) [141] }\end{array}$ & $4-80$ & $100-350$ & $11-15$ & Inkjet printing \\
\hline $\begin{array}{l}\text { NovaCentrix ICI-003 } \\
\text { (copper inkjet) } \\
{[141]} \\
\end{array}$ & 4.3 & PulseForge $^{\mathrm{TM}}$ & $1-6.5$ & piezo-inkjet printing \\
\hline $\begin{array}{l}\text { NovaCentrix JS-015 } \\
\text { (silver nanoparticles) [141] }\end{array}$ & 2.4 & Thermal/PulseForge $^{\mathrm{TM}}$ & $1-5$ & piezo-inkjet printing \\
\hline $\begin{array}{l}\text { InkTec IJ-010 } \\
\text { (soluble silver cluster \& complex) } \\
{[141]}\end{array}$ & 4.2 & $130-150$ & $9-15$ & Inkjet printing \\
\hline $\begin{array}{l}\text { NanoMas NTS05 } \\
\text { (nanosilver ink) } \\
{[141]} \\
\end{array}$ & 2.4 & $70-150$ & $1-10$ & - \\
\hline $\begin{array}{l}\text { Polymer thick film (PTF) ink } \\
{[142]}\end{array}$ & 80 & 120 & $20000-30000$ & Screen printing \\
\hline
\end{tabular}

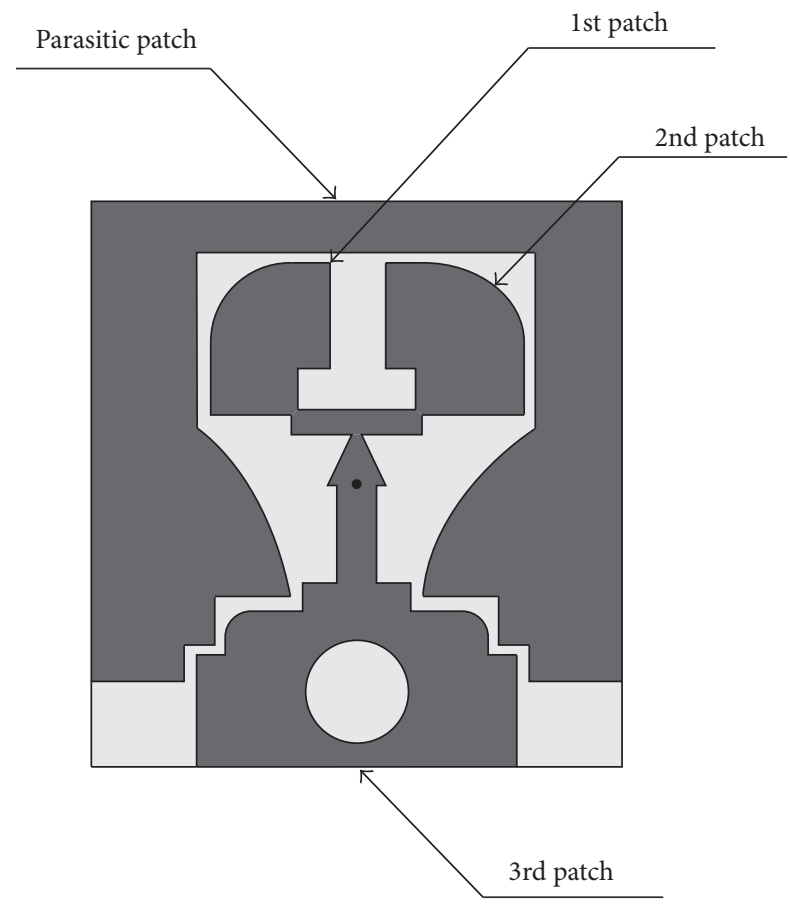

FIgURE 42: Geometry of the UWB all-textile antenna with full ground plane for off-body WBAN communications [144]. The three patches and the parasitic one forming the wearable antenna are indicated in the figure. The black dot point indicates the pin of the coaxial probe. Dark gray upper conductive textile and light gray dielectric textile substrate.

NY, USA) is a $0.17 \mathrm{~mm}$ thick flexible conductive textile having a conductivity of about $1.18 \times 10^{5} \mathrm{~S} / \mathrm{m}$, while the felt is a thermally isolating material with thickness $3 \mathrm{~mm}$, a relative permittivity of 1.45 , and a loss tangent of 0.044 .
The antenna is formed by three main radiators connected in parallel by means of a short transmission line. An additional parasitic radiator, excited through a capacitive coupling, surrounds the main radiators. The operating frequencies of the main radiators are designed to be closely spaced, enabling a combined wideband resonance for the ATA-FGP. The capacitive coupling between the active patches and the surrounding parasitic radiator contributes to increase the antenna bandwidth. Using said assembly an operating band between 3 and $10 \mathrm{GHz}$ is obtained. The ATA-FGP exhibits a wide beam in the boresight direction, while the presence of the full ground drastically reduces the energy emission toward the user body. Antenna gain ranges between 5.1 and $6.3 \mathrm{dBi}$ when the antenna is on the chest and between 3.2 and $6.9 \mathrm{dBi}$ when it is on the back of a human body.

A different all-textile antenna, composed of Shieldit for the conductive part and of felt for the dielectric part, was proposed in [145] for WBAN and wearable computing. The structure consists of two patches and a ground plane implemented on two layers of $2 \mathrm{~mm}$ thick substrate. A full ground plane on the lower layer shields the user against on-body radiation and improves the quality of the radiated/received UWB signal. However, because full ground planes typically limit the impedance bandwidth of printed monopole antennas, a parasitic circular metal patch between the radiating octagonal patch and the full ground plane was employed to enhance the antenna bandwidth. In particular, the circular patch, connected by a thin strip to the ground, is inserted between two layers of dielectric material to prevent direct contact with the octagonal patch and the ground plane, realizing in this way a stacked configuration (see Figure 43). A step is introduced in the ground plane near the $50 \Omega \mathrm{CPW}$ feeding line to enhance the antenna impedance bandwidth.

The conductive parts of the antenna formed by Shieldit, a conductive textile from Less EMF Inc., have thickness of 

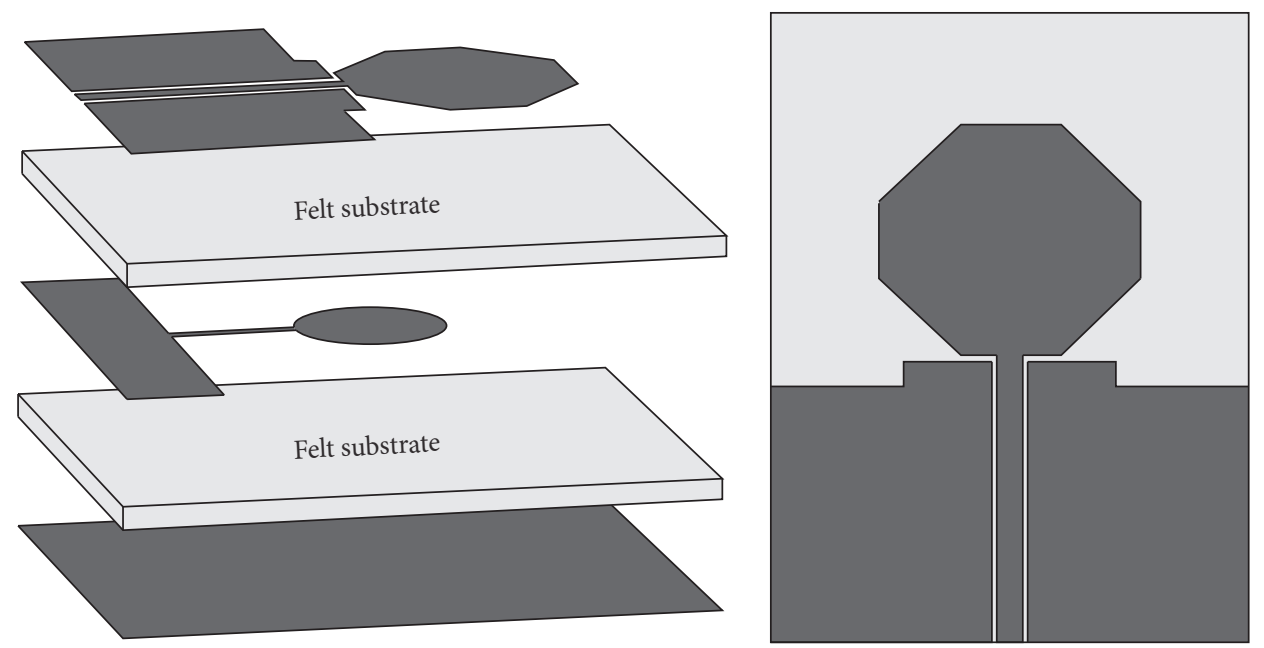

FIGURE 43: Geometry of an all-textile UWB antenna with low back radiation for WBAN applications [145]. Dark gray conductive textile and light gray dielectric textile substrate.

$0.17 \mathrm{~mm}$ and an estimated conductivity of $1.18 \times 10^{5} \mathrm{~S} / \mathrm{m}$, while the felt substrate layers have a relative permittivity of 1.45 and a loss tangent of 0.044 . The overall dimensions of the antenna are $80 \mathrm{~mm} \times 61 \mathrm{~mm} \times 4.51 \mathrm{~mm}$, corresponding to about $0.85 \lambda_{0} \times 0.65 \lambda_{0} \times 0.05 \lambda_{0}$ at the lowest operating frequency of $3.18 \mathrm{GHz}$. The antenna covers the UWB bandwidth ranging between $3.18 \mathrm{GHz}$ and $11 \mathrm{GHz}$, achieving a maximum gain of about $7.2 \mathrm{dBi}$ at $9 \mathrm{GHz}$ with unidirectional radiation patterns. Measurements performed with the antenna placed on the arms and chest of a human being have highlighted excellent radiative performances with simulated SAR values lower than $2 \mathrm{~W} / \mathrm{kg}$ averaged over $10 \mathrm{~g}$ of tissue when excited by signal of $0.5 \mathrm{~W}$ (rms). Fidelity factors and pulse distortions have been found comparable with those of other textile antennas proposed in literature. The analysis and design of the antenna have been performed using the full-wave FITbased CST Microwave Studio [25].

A dual-band wearable textile antenna based on a substrate integrated waveguide technology (SIW) featuring an integrated flexible solar harvesting system, consisting of a flexible solar cell, a power management system, and energy storage, has been presented in [146]. The antenna, working in the $2.4-2.4835 \mathrm{GHz}$ industrial, scientific, and medical band and the 2.5-2.69 GHz 4G LTE band 7, can be used to monitor rescue workers during interventions and patients in hospitals and home-care. The proposed antenna consists of two layers of conductive material consisting of copper polyester Taffeta, with a surface resistivity $0.18 \Omega / \mathrm{m}^{2}$, separated by a layer of expanded rubber protective foam with $\varepsilon_{r}=1.49$, loss tangent 0.016 , and thickness $3.94 \mathrm{~mm}$. The backed cavity is realized by using a row of equally spaced flat flange copper tube eyelets along each side of the rectangular cavity. The cavity is split into two halves (see Figure 44) by a nonresonant rectangular slot. The dimensions of the slot and both parts of the cavity were carefully chosen so as to excite simultaneously two hybrid modes responsible for the antenna bandwidth. Finally, a thin and flexible solar cell is placed on the upper part of the first cavity (see Figure 44), while on the rear side of the antenna is housed the power management system (PMS) so as to prevent antenna radiation into the electronic circuitry. The experimental measurements performed on an antenna prototype have shown that the antenna exhibits a gain ranging between 4.7 and $5.1 \mathrm{~dB}$, a wide bandwidth, and a high front-to-back ratio. Moreover, its high robustness against bending, the low influence of the human body, and the flexible structure make the proposed antenna particularly suitable for wearable applications. The analysis and design of the antenna have been performed using the full-wave FITbased CST Microwave Studio [25].

Other examples of wearable antennas, not reported for the sake of brevity, can be found in [113, 115, 147-149], while examples involving SIW antennas can be found in [150-154].

\section{Dielectric Resonator Antennas}

High permittivity dielectric resonators (DRs) have been employed in the past to develop low losses microwave circuits [155], filters [156], and oscillators [157]. Although DRs were mainly conceived for said applications, in the early 1980s-1990s Long and others [158] proposed for the first time the use of dielectric resonators as high-efficiency radiating structures. In particular, they employed a magnetic walls model to analyze cylindrical resonators, having a dielectric constant lower than that typically used to excite high $Q$ resonant processes [158]. Later, they analyzed the electromagnetic behavior of rectangular and hemispherical dielectric resonators $[159,160]$. In particular, whereas for the rectangular resonator the same model used to analyze the cylindrical resonator was employed, for the hemispherical resonator a rigorous full-wave solution was adopted to identify the resonance frequencies of its resonant modes [160]. The geometries of the above-mentioned DRAs are depicted in Figure 45.

Later, in the 1990s Leung et al. [161] have deepened the research activities on the DRAs proposing some excitation 


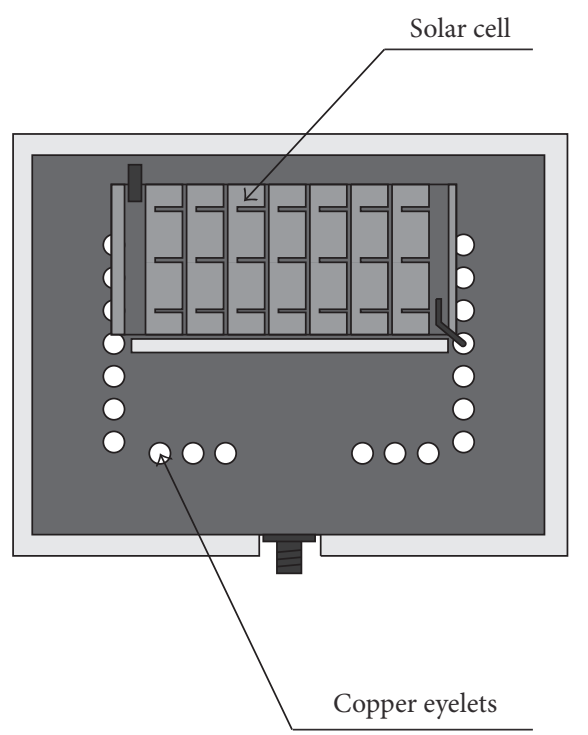

(a)

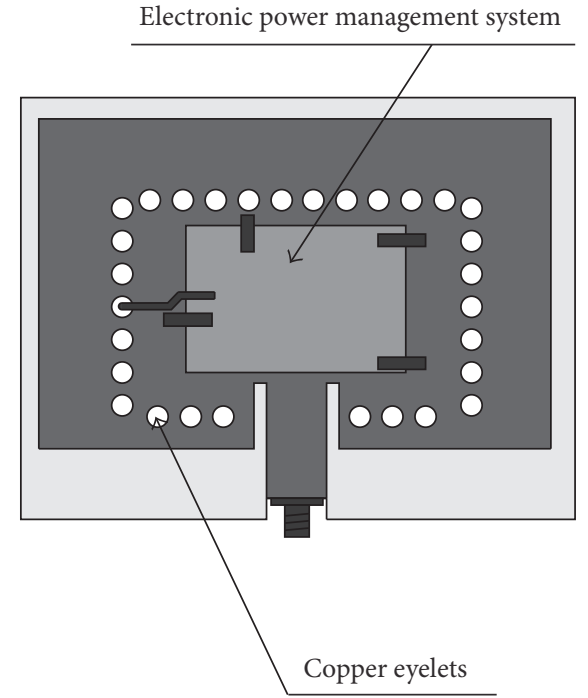

(b)

FIGURE 44: Geometry of the SIW cavity-backed slot wearable textile antenna with the integrated energy harvesting hardware [146]. Dark gray conductive textile and light gray dielectric textile substrate. The conductors employed to connect the solar cell and PMS are indicated in the figure. Top view (a) and bottom view (b).

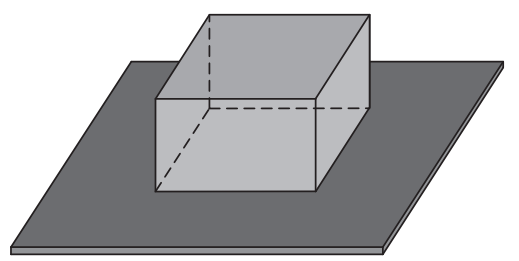

(a)

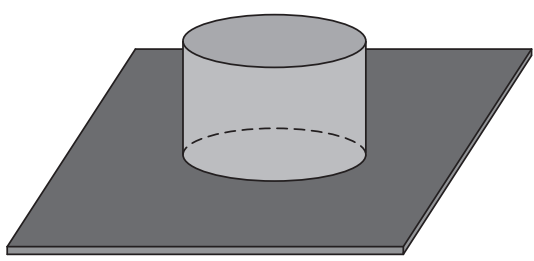

(b)

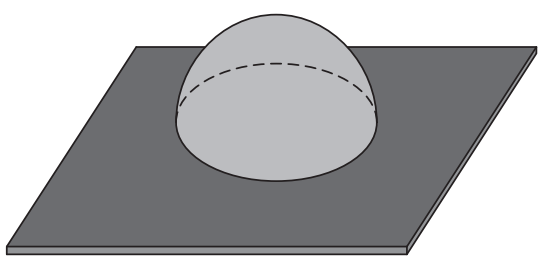

(c)

FIgURE 45: Rectangular (a), cylindrical (b), and hemispherical (c) DRA on a metallic ground plane.

techniques aimed at the integration of the antennas with the RF circuitry. In addition, they addressed the problem of the full-wave modeling using the MoM [162] and the FDTD [163] techniques aimed at assessing the quality factor, the input impedance, and antenna radiation patterns.

MoM techniques were also employed by Junker et al. to analyze structures having rotational symmetry (BOR) in order to reduce the computational burden necessary to the computation of the reflection coefficients [164] and of the radiation diagrams [165]. These authors also used a dyadic Green's function approach to assess the frequency behavior of the input impedance and of the quality factor of hemispherical resonators, and they extended the MoM analysis to the assessment of the electromagnetic coupling of bodies of revolution (BOR) with non-BOR feeding structures [166]. In addition, they expanded the MoM technique to determine the power delivered to cylindrical resonant structures excited by a narrow slot in a rectangular waveguide [167].

Finally, Mongia et al. have deepened the study of the characteristics of some geometries (dielectric ring resonator antenna [168], triangular resonator [169]), providing approximate formulas for the calculation of the resonance frequency and the quality factor, as well as a standardization of the nomenclature of the modes excited in dielectric resonators and antennas [170].

The results obtained until the 2000s have allowed identifying several geometries, excitation techniques, and some heuristic formulas [170] useful to the assessment of the working frequencies of the resonators most commonly employed in practical applications [1, 158-160].

However, these structures were characterized by limited fractional bandwidths not compatible with the evolution of the modern wireless communication systems. For this reason, the research activities carried out in the last decades of the 2000s have been focused on the ways for widening the band, for increasing the gain, and for improving the quality of the linear or circular polarization, as well as for the miniaturization and integration of the radiating structures into portable devices. For the sake of brevity, in the following an overview of the most significant papers concerning the aforementioned design aspects is provided, while in Tables 5 and 6 the excitation techniques and typical high performance dielectric materials together with their suppliers are listed, respectively. 
TABLE 5: Techniques for DRAs excitation.

\begin{tabular}{|c|c|c|}
\hline $\begin{array}{l}\text { Feeding } \\
\text { technique }\end{array}$ & Characteristics & Reference \\
\hline Waveguide & $\begin{array}{l}\text { High coupling with } \\
\text { high permittivities } \\
\text { DRAs achieved } \\
\text { through a slot in the } \\
\text { waveguide wall. }\end{array}$ & [171-173] \\
\hline $\begin{array}{l}\text { Aperture } \\
\text { coupled }\end{array}$ & $\begin{array}{l}\text { Used to excite a DRA } \\
\text { using a microstrip } \\
\text { line (a reduced } \\
\text { surface waves level is } \\
\text { excited). }\end{array}$ & [174-178] \\
\hline $\begin{array}{l}\text { Microstrip } \\
\text { line }\end{array}$ & $\begin{array}{l}\text { Used to excite high } \\
\text { permittivity DRAs. } \\
\text { For low permittivity } \\
\text { DRAs, tall microstrip } \\
\text { lines (TMLs) with a } \\
\text { dielectric support are } \\
\text { used to enhance the } \\
\text { coupling }[115,179] .\end{array}$ & [179-181] \\
\hline $\begin{array}{l}\text { Electric } \\
\text { probe }\end{array}$ & $\begin{array}{l}\text { Used to excite a DRA } \\
\text { using a coaxial cable. }\end{array}$ & [182-187] \\
\hline Strip-fed & $\begin{array}{l}\text { Used to excite a DRA } \\
\text { when broadband } \\
\text { antenna behavior } \\
\text { needs to be achieved. }\end{array}$ & [188-190] \\
\hline
\end{tabular}

10.1. Broadband DRA. Since broadband communication systems allow offering a variety of wireless services in a single mobile device [21, 182], as well as higher data rate [191], several studies useful to provide guidelines to design wideband dielectric resonator antennas have been proposed in the scientific literature $[192,193]$. In fact, the antenna geometry, the suitable choice of the dielectric materials, as well as that of the appropriate feeding techniques, can lead to the excitation of specific resonant modes characterized by low values of the antenna $Q$-factor. In particular, a new class of super shaped DRAs (see Figure 46), making use of low dielectric permittivity plastic materials [polyvinyl chloride (PVC) with $\varepsilon_{r}=2.8$ ] was proposed in [183] with the aim to obtain a high operating bandwidth (exceeding 70\%), an antenna gain ranging from 5 up to $10 \mathrm{dBi}$, and an excellent linear/circular polarization purity. The antennas belonging to the mentioned class may be advantageously used to operate in indoor environments, as well as on access points of satellite terminal receivers. The geometrical dimensions of the considered DRAs are of $100 \mathrm{~mm} \times 27 \mathrm{~mm}$ (about a wavelength within the material forming the DRA at the frequency of $7.5 \mathrm{GHz}$ ). The antenna analysis and design were carried out by means of the full-wave FIT-based CST Microwave Studio [25].

A different design solution has been proposed in [194]. This solution makes use of three cylindrical resonators having different dimensions and dielectric permittivities, stacked along the symmetry axis (boresight direction). The resulting antenna (see Figure 47), characterized by a broadband behavior $(\mathrm{FBW}=26 \%$ ) which extends from 5.4 to $7.5 \mathrm{GHz}$, for the

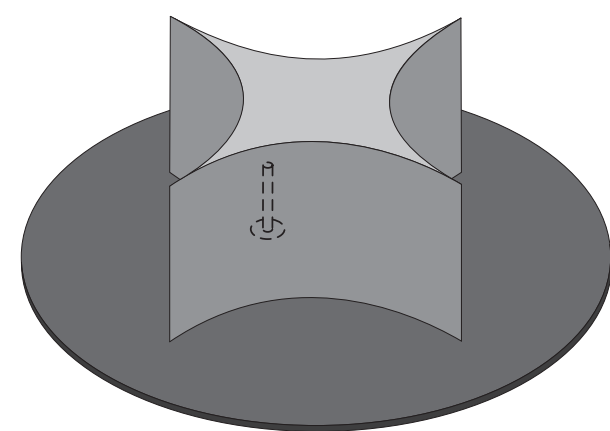

FIGURE 46: Geometry of the plastic-based super shaped dielectric resonator antenna [183].

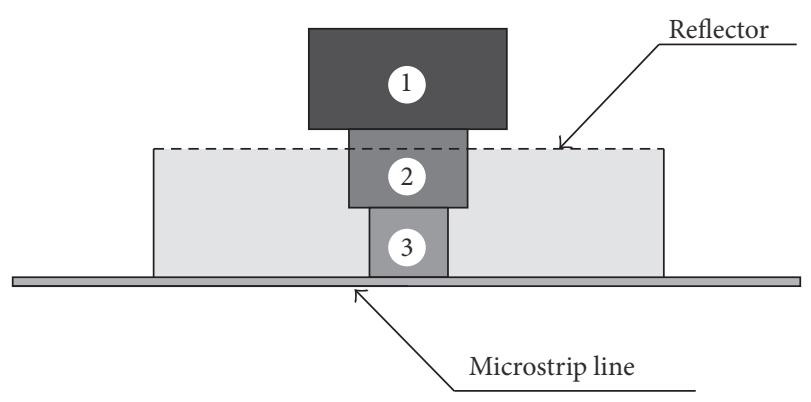

FIGURE 47: Geometry of the wideband high-gain stacked cylindrical dielectric resonator antenna [194]. The microstrip line adopted to excite the antenna is also indicated in the figure.

simulated, and from 5.4 to $7 \mathrm{GHz}$, for the measured, exhibits a high-gain ranging from about 9 up to $11 \mathrm{dBi}$.

The band widening is obtained by merging the fundamental resonant frequencies of the three DRs whose dielectric materials are the Rogers RT/Duroid 6010 with $\varepsilon_{r 1}=10.2$, the Rogers RT/Duroid 6006 with $\varepsilon_{r 2}=6.15$, and Rogers RT/Duroid 5880 with $\varepsilon_{r 3}=2.2$. To increase the antenna gain the stacked DRs are surrounded by a cylindrical metal reflector. The antenna, whose ground plane dimensions are $120 \mathrm{~mm} \times 120 \mathrm{~mm}$ (about $2.3 \lambda_{0} \times 2.3 \lambda_{0}$ ) and whose height is equal to about $0.65 \lambda_{0}$ (where $\lambda_{0}$ is the free-space wavelength at the central frequency of $6.5 \mathrm{GHz}$ ), is excited by a microstrip line coupled to the DRs assembly by means of a slot in the ground plane. The analysis and design have been performed using the full-wave FEM-based HFSS [8] and FIT-based CST Microwave Studio [25]. The radiation diagrams show a good angular behavior with a very limited level of crosspolarization along the boresight direction $(-40 \mathrm{~dB})$. The proposed solution presents a front-to-back ratio of about $22 \mathrm{~dB}$ at $6 \mathrm{GHz}$.

As discussed above, the adoption of low permittivity dielectric materials can be useful to extend the antenna bandwidth. To this end, in [1] some dielectric products having low dielectric permittivity are reported. In addition, in the same paper some geometrical shapes and some feeding systems useful for the excitation of circular polarization [195] (see Figure 48), as well as for the excitation of the higher order modes useful to increase the antenna gain or to confer 
TABLE 6: Dielectric materials for DRAs realization.

\begin{tabular}{|c|c|c|c|c|}
\hline Company & Material & Permittivity & \multicolumn{2}{|c|}{ Applications } \\
\hline \multirow[t]{2}{*}{$\begin{array}{l}\text { Emerson \& } \\
\text { Cuming }\end{array}$} & $\begin{array}{l}\text { ECCOSTOCK HiK500F } \\
\quad \text { (low-loss) }\end{array}$ & $\begin{array}{c}3-30 \pm 3 \% \\
( \pm 10 \% \text { for dielectric } \\
\text { constant }>16)\end{array}$ & \multirow{2}{*}{\multicolumn{2}{|c|}{$\begin{array}{l}\text { Outdoor applications, cavity tuning probes, patch } \\
\text { antennas, dielectric lens antennas, and } \\
\text { dielectric support pieces }\end{array}$}} \\
\hline & ECCOSTOCK HiK & $3-15 \pm 3 \%$ & & \\
\hline \multirow{11}{*}{ Trans-Tech $^{\mathrm{TM}}$} & D-100 Titania & $100.0 \pm 5 \%$ at $6.0 \mathrm{GHz}$ & \multirow{9}{*}{\multicolumn{2}{|c|}{$\begin{array}{l}\text { High-frequency DRs, patch antenna, matching } \\
\text { structures for circulators and isolators, and } \\
\text { miscellaneous support structures }\end{array}$}} \\
\hline & D-15 Mg-Ti (ceramic) & $15.0 \pm 0.5$ at $9.4 \mathrm{GHz}$ & & \\
\hline & D-16 Mg-Ti (ceramic) & $16.0 \pm 0.5$ at $9.4 \mathrm{GHz}$ & & \\
\hline & D-38 Ba-Ti (ceramic) & $37.0 \pm 5 \%$ at $6.0 \mathrm{GHz}$ & & \\
\hline & $\begin{array}{l}\text { D-4 cordierite } \\
\text { (hard ceramic) }\end{array}$ & $4.5 \pm 0.2 \%$ at $9.4 \mathrm{GHz}$ & & \\
\hline & D-50 Ba-Ti & $50.0 \pm 5 \% 6.0 \mathrm{GHz}$ & & \\
\hline & $\begin{array}{l}\text { DS- } 6 \text { forsterite } \\
\quad \text { (ceramic) }\end{array}$ & $6.3 \pm 0.3 \%$ at $9.4 \mathrm{GHz}$ & & \\
\hline & Magnesium aluminum titanate & $9-9.5 \pm 0.3 \%$ at $9.4 \mathrm{GHz}$ & & \\
\hline & (easy to machine ceramic) & $10-14 \pm 0.5 \%$ at $9.4 \mathrm{GHz}$ & & \\
\hline & $\begin{array}{l}\text { Magnesium calcium titanate } \\
\text { (easy to machine ceramic) }\end{array}$ & $\begin{array}{c}18-140 \\
\text { (between } \pm 3 \% \text { and } \pm 10 \% \text { ) }\end{array}$ & \multirow{2}{*}{\multicolumn{2}{|c|}{$\begin{array}{l}\text { Cellular base-station filters and combiners, } \\
\text { broadcast satellite receivers, and } \\
\text { WiMAX filters }\end{array}$}} \\
\hline & $\begin{array}{l}\text { Temperature stable ceramic } \\
\text { dielectrics }\end{array}$ & $\begin{array}{c}21.5-73 \\
\text { (between } \pm 0.5 \% \text { and } \pm 1 \% \text { ) }\end{array}$ & & \\
\hline \multirow[t]{2}{*}{ Magnetics Group } & $\begin{array}{l}\text { From K- } 4 \text { to } \mathrm{K}-250 \\
\quad \text { (ceramic) }\end{array}$ & $4.3-250 \pm 5 \%$ & \multicolumn{2}{|c|}{$\begin{array}{c}\text { Patch antennas for automotive and GPS, dielectric rod } \\
\text { antennas, ceramic supports, sleeves for ferrites, } \\
\text { waveguide filters, phase shifters and tuning elements, } \\
\text { and sputtering targets }\end{array}$} \\
\hline & From DR-30 to DR-80 & $\begin{array}{l}29.5-46.5 \pm 1 \% \\
80 \pm 2 \%\end{array}$ & \multicolumn{2}{|c|}{$\begin{array}{l}\text { Base stations, filters and combiners, broadcast satellite } \\
\text { receivers, wireless, and cable TV }\end{array}$} \\
\hline \multirow{8}{*}{$\begin{array}{l}\text { Murata } \\
\text { Manufacturing Co. }\end{array}$} & U series & $36-40 \pm 0.5 \%$ & $1.5-12.5 \mathrm{GHz}$ & \multirow{8}{*}{$\begin{array}{l}\text { (i) Satellite antenna } \\
\text { (ii) Burglar alarms } \\
\text { (iii) Multichannel microwave } \\
\text { communications systems } \\
\text { (iv) Radar systems } \\
\text { (v) Speed guns } \\
\text { (vi) Mobile phone } \\
\text { (vii) CATV converters }\end{array}$} \\
\hline & M series & $37-40 \pm 1 \%$ & $1.5-12.5 \mathrm{GHz}$ & \\
\hline & V series & $33-36 \pm 0.5 \%$ & $2.9-13.2 \mathrm{GHz}$ & \\
\hline & R series & $29-31 \pm 0.8 \%$ & $4.6-24.2 \mathrm{GHz}$ & \\
\hline & B series & $27-29 \pm 0.5 \%$ & $4.8-25.9 \mathrm{GHz}$ & \\
\hline & E series & $24-25 \pm 0.4 \%$ & $\begin{array}{l}8.4 \mathrm{GHz}-25 \mathrm{GHz} \\
\text { (also above } 25 \mathrm{GHz} \\
\text { applications) }\end{array}$ & \\
\hline & F series & $23-24 \pm 0.5 \%$ & $\begin{array}{c}10 \mathrm{GHz}-25 \mathrm{GHz} \text { (also } \\
\text { above } 25 \mathrm{GHz} \\
\text { applications) }\end{array}$ & \\
\hline & $Z$ series & $6.4 \pm 0.6 \%$ & $\begin{array}{l}\text { Support of dielectric } \\
\text { resonators }\end{array}$ & \\
\hline \multirow{7}{*}{ Token Company } & TE21 & $19-22$ & \multirow{2}{*}{$\begin{array}{l}\mathrm{C} / \mathrm{X} / \mathrm{Ku} / \mathrm{K} / \mathrm{Ka} \text { band } \\
\mathrm{S} / \mathrm{C} / \mathrm{X} / \mathrm{Ku} \text { band }\end{array}$} & \multirow{7}{*}{$\begin{array}{c}\text { (i) LNB } \\
\text { (ii) Radar detectors } \\
\text { (iii) DRAs } \\
\text { (iv) Cable TV } \\
\text { (v) Anticollision sensors } \\
\text { (vi) Filters, duplexer, and } \\
\text { combiners } \\
\text { (vii) Cellular base filters, } \\
\text { duplexer, and combiners }\end{array}$} \\
\hline & TE30 & $29-30$ & & \\
\hline & & & L/S/C/X band & \\
\hline & TE36 & $35-37$ & & \\
\hline & TE45 & $44-46$ & $\mathrm{UHF} / \mathrm{L} / \mathrm{S} / \mathrm{C} / \mathrm{X}$ band & \\
\hline & TE80 & $79-81$ & $\mathrm{UHF} / \mathrm{L} / \mathrm{S} / \mathrm{C}$ band & \\
\hline & TE90 & $89-91$ & $\mathrm{UHF} / \mathrm{L} / \mathrm{S}$ band & \\
\hline Kyocera & Microwave dielectric ceramics & $\begin{array}{c}\text { 9.7-141 } \\
\text { (between } \pm 0.5 \% \text { and } \pm 3 \% \text { ) }\end{array}$ & \multicolumn{2}{|c|}{ Dielectric resonator, satellite broadcast, and base station } \\
\hline
\end{tabular}




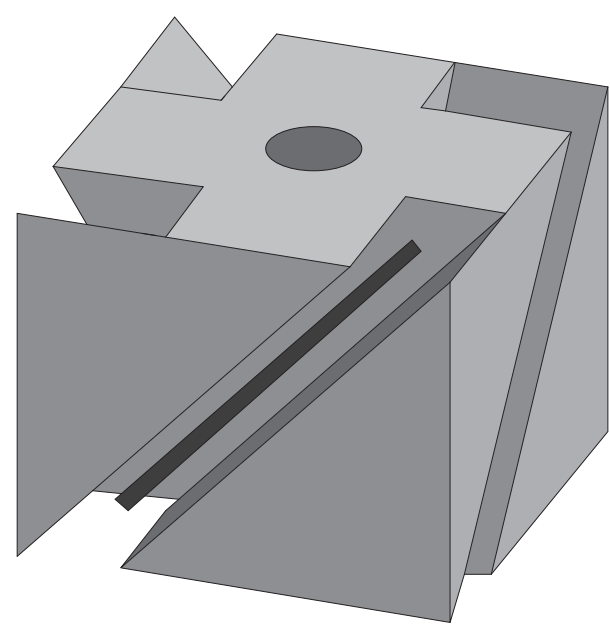

(a)

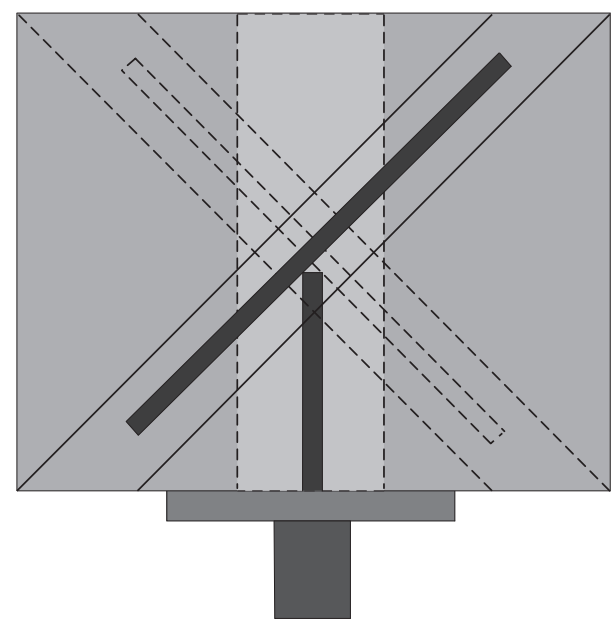

(b)

FIGURE 48: DRA working in circular polarization. The slits on the DR excite the circular polarization, while the metal strips (drawn in black) are used to increase the operating bandwidth of the axial ratio triggering a circular polarization that goes to overlap to that radiated by the DR [195]. Tridimensional view (a) and front view (b).

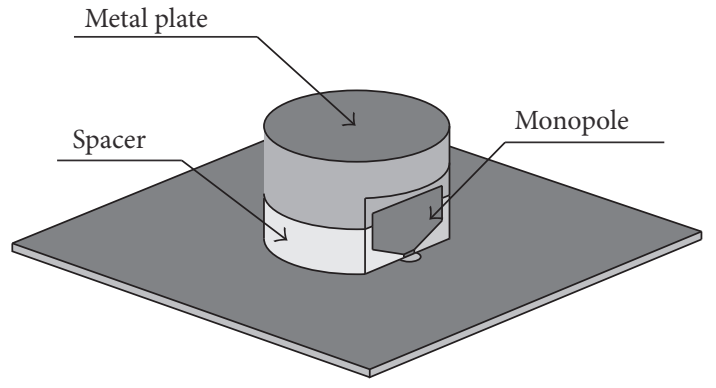

(a)

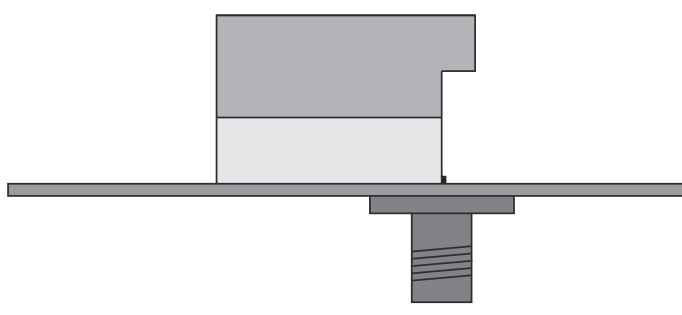

(b)

FIGURE 49: A dielectric resonator antenna excited by an electric probe having a trapezoidal shape [197]. Tridimensional view (a) and lateral view (b). The DR region with the notch is that where the strip-fed system used to excite the DRA is placed.

to the DR a dual or a multiband behavior, are discussed. This information can be beneficial whenever it is necessary to compact the communication system conferring to the DR a filtering [196] and radiant behavior [1].

In addition to geometry and to dielectric materials, the antenna excitation system may have a significant impact on the bandwidth of the radiating structure. In particular, an antenna composed of a dielectric spacer, made of foam having a dielectric constant close to one, on which a DR is placed, having a dielectric constant $\varepsilon_{r}=9.8$ (adopted dielectric material TMM10i), and with a metal plate placed on the top surface of the DR, has been proposed in [197]. The DR is excited by means of a trapezoidal monopole patch, attached to the side surface of the resonator on which a suitable notch is realized, connected to an SMA connector. The air-region, forming the spacer, behaves as a magnetic wall on which is mounted the dielectric resonator loaded with a top metal surface. The electromagnetic analysis was performed using the full-wave FEM-based HFSS [8]. The prototype antenna has a frequency band ranging between 2.9 and $6.7 \mathrm{GHz}$
$(\mathrm{FBW}=75 \%)$ with a gain varying between 4.8 and $6.5 \mathrm{dBi}$. This result was achieved realizing a bevel on the exciting monopole, which allows an effective impedance matching with the coaxial line (see Figure 49). The dimensions of the ground plane are about $50 \mathrm{~mm} \times 50 \mathrm{~mm}$ while the total height of the DRA is of about $15 \mathrm{~mm}$. The structure has dimensions of about $0.48 \lambda_{0} \times 0.48 \lambda_{0} \times 0.14 \lambda_{0}$, where $\lambda_{0}$ is the wavelength at the lower frequency.

10.2. High-Gain DRAs. Typical dielectric resonator antennas exhibit gains of about 4-5 dBi (suitable for indoor coverage) which may be not suitable for some applications such as those concerning wireless base stations and satellite communications. In particular, radio base stations operating in outdoor environments may require gains that vary in the range of about 3 to $30 \mathrm{dBi}$ depending on the type of radio links and coverage requirements [198], while for satellite applications such gains may oscillate between $12 \mathrm{dBi}$ (or lower for small satellite applications) and $50 \mathrm{dBi}$ (for high-gain satellite applications) [199]. Also the working frequency can 
be important because in the frequency range of $60 \mathrm{GHz}$ the atmospheric absorption of electromagnetic waves is particularly significant and consequently said frequency is adopted only for short-range wireless communications making use of high-gain antennas [174].

One of the main expedients used for increasing the antenna gains in a limited frequency band consists in the excitation of higher order modes [174, 184, 200-202]. In fact, when these modes are excited, the antenna appears electrically bigger and thus the realized gains are higher. Furthermore, because the realization of an array is not required, the complexity and size of the antenna are more limited. In addition, the mutual coupling between the array elements is finally avoided [200]. In particular, a high-gain rectangular dielectric resonator antenna, backed by a ground plane with a suitable slit excited by a microstrip line, has been proposed in [202]. The gain increase is achieved by exciting the resonator on the higher order modes. In this way a higher gain of about $5 \mathrm{~dB}$ compared to that obtained by a conventional excitation of the fundamental resonant mode is obtained. Three different DRAs, of relative dielectric permittivity 10 and $\tan \delta=0.02$, having increasing heights, are used to excite modal configurations of higher order, highlighting how the increase of the modal order as well as that of the resonator's height results in an increase of the antenna gain. The ground plane of the DRA is approximately $100 \mathrm{~mm} \times 150 \mathrm{~mm}\left(3.3 \lambda_{0} \times 5 \lambda_{0}\right.$, where $\lambda_{0}$ is the freespace wavelength). A homemade full-wave FDTD numerical technique was adopted to analyze the antennas. Gains of about $10 \mathrm{dBi}$ and $\mathrm{FBW}=7.5 \%$ are obtained in the resonator having a height of about $30 \mathrm{~mm}\left(\lambda_{0}\right)$.

In addition to the use of techniques aimed at the excitation of suitable higher order modes, the use of metal reflectors and of appropriate design solutions allows a considerable increase of the antenna gain $[185,187,194]$. In particular, the simultaneous use of stacked DRAs and of a cylindrical metal reflector placed in proximity of the DRAs (see Figure 47) allows achieving a gain of about $11 \mathrm{dBi}$ with a FWB $=26 \%$ and a front-to-back ratio of about 17-30 dB [194].

Higher radiative performances, on a higher operating bandwidth (exceeding 65\% fractional bandwidth), are reached using a suitably shaped metal reflector and equipping the DRA with a top-mount spherical cap-lens (see Figure 50) useful to focus the RF energy in the boresight direction [185]. The materials chosen to realize the $\operatorname{DR}\left(\varepsilon_{r}=3\right)$ and the dielectric lens $\left(\varepsilon_{r}=4\right)$ belong to the ECCOSTOCK ${ }^{\circledR}$ HiK 500F family, featuring a loss tangent less than 0.002 in the frequency range $1-10 \mathrm{GHz}$. The antenna has a diameter of about $114 \mathrm{~mm}$ (equal to about $2.6 \lambda_{0}$ at the central working frequency) and a height of about $48 \mathrm{~mm}\left(\lambda_{0}\right)$. This design solution allows achieving a high-gain (exceeding $15 \mathrm{dBi}$ ), excellent linear/circular polarization purity, and a high front-to-back ratio (better than $20 \mathrm{~dB}$ ). Said characteristics make the antenna suitable to be successfully employed in indoor/outdoor wireless communication systems as well as for satellite terminal receivers, for instance, to effect a transponder function between $\mathrm{WiFi}$ and satellites in the socalled $8 / 7 \mathrm{GHz} \mathrm{X}$ band. In addition, it can also be advantageously used in UWB wireless applications working with

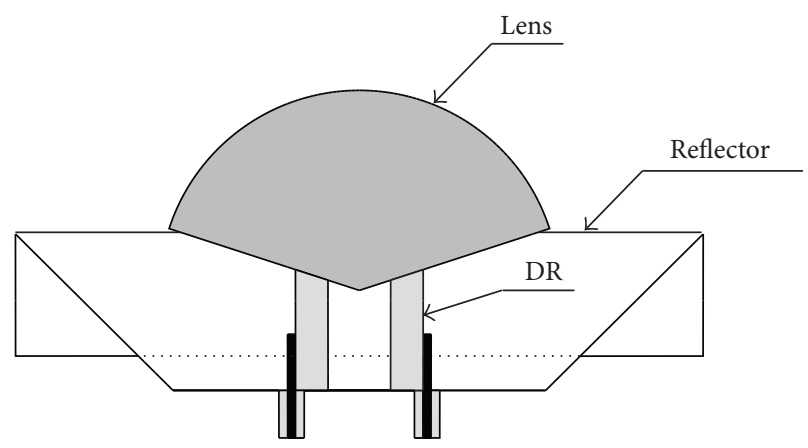

FIGURE 50: Geometry of the high-gain mushroom-shaped dielectric resonator antenna in the vertical symmetry plane of the antenna [185]. The DR, the lens, the reflector, and two of the four coaxial probes useful to excite the circular polarization are reported in the figure.

pulse signals. The antenna, making use of low permittivity dielectrics, can be realized with lightweight commercialgrade materials and thus may be suitable for space/airborne applications at a reasonable manufacturing cost and complexity. The full-wave FIT-based CST Microwave Studio [25] has been employed to design and characterize the antenna, while the singularity expansion method has been adopted to express the antenna response to arbitrary excitation waveforms.

10.3. Compact DRA. Tridimensional radiating structures may not be suitable to be integrated in portable devices where antennas of small size and low-profile are preferable. For this reason new geometries and excitation techniques for achieving more compact dielectric resonator antennas were proposed in $[196,203]$. In particular, a DRA formed by a rectangular DR, having a dielectric permittivity 9.2 (commercial product TMM10), spaced from the ground plane by means of a dielectric insert of permittivity $\varepsilon_{r}=2.2$ (commercial product Rogers RT/Duroid 5880), and excited by a probe placed inside the DRA on the side wall of which a metal surface is placed (see Figure 51), was presented in [203]. The antenna, of size $40 \mathrm{~mm} \times 40 \mathrm{~mm} \times 15.2 \mathrm{~mm}\left(0.41 \lambda_{0} \times\right.$ $0.41 \lambda_{0} \times 0.16 \lambda_{0}$, where $\lambda_{0}$ is the free-space wavelength at $f=$ $3.1 \mathrm{GHz}$ ), analyzed by means of the full-wave commercial software tools FIT-based CST Microwave Studio [25] and FEM-based HFSS [8], is suitable to cover the UWB band (3.1$10.6 \mathrm{GHz}$ ) with an antenna gain ranging between 4 and the $6.8 \mathrm{dBi}$.

A more compact and thin DRA (see Figure 52), of size $12 \mathrm{~mm} \times 30 \mathrm{~mm} \times 6 \mathrm{~mm}\left(0.124 \lambda_{0} \times 0.31 \lambda_{0} \times 0.062 \lambda_{0}\right.$, where $\lambda_{0}$ is the free-space wavelength at $3.1 \mathrm{GHz}$ ), suitable for handheld devices, has been recently proposed in [196]. The antenna, having UWB characteristics, ensures low emissions in the WiMAX and WLAN frequency bands, so reducing the electromagnetic interferences (EMIs) with such systems.

The radiating structure, composed of two identical rectangular DRA of permittivity $\varepsilon_{r}=10$ and $\tan \delta=0.002$, is excited by a microstrip feeding line, realized on a RO3003 having $\varepsilon_{r}=3$ and thickness $0.75 \mathrm{~mm}$, equipped with three teeth. The filters employed for the rejection of the 


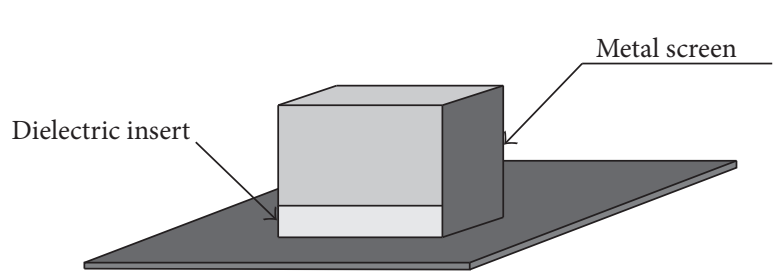

(a)

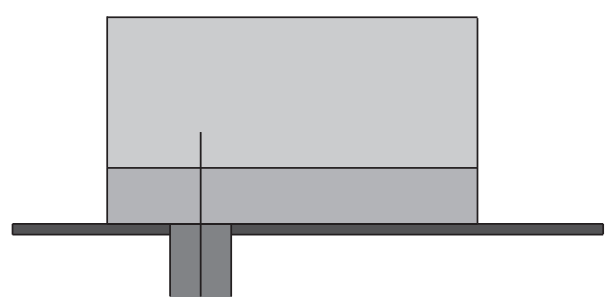

(b)

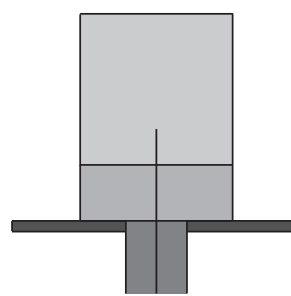

(c)

FIGURE 51: A compact rectangular dielectric resonator antenna with a low permittivity insert placed between the ground plane and the DR [203]. Tridimensional view (a), front view (b), and lateral view (c).

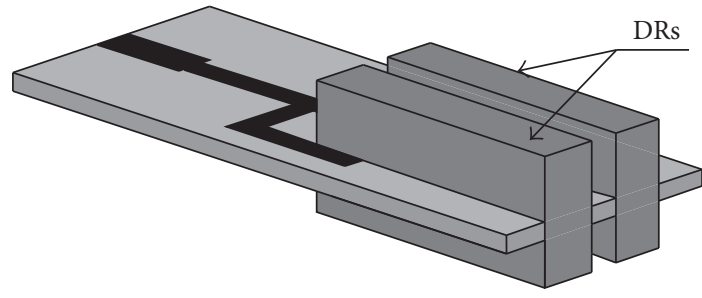

FIGURE 52: Tridimensional view of a compact UWB DRA with dualband-rejection characteristics for WiMAX/WLAN bands [196].

WiMAX and WLAN bands are composed of a T-shaped microstrip resonator and by a rectangular slit integrated in the ground plane. The low dielectric permittivity adopted for the realization of the DRA allows obtaining a wide operating band (equal to approximately $122 \%$ ) with limited geometric dimensions. The antenna characteristics were carried out by means of the full-wave FIT-based CST Microwave Studio [25] and with the FEM-based HFSS [8].

\section{UWB Antennas with Band-Notched Characteristics}

Techniques useful to limit the interference between UWB and other communication systems operating in the same environment (WLAN, WiMAX, etc.) are presented in this section. These techniques employ suitable filter systems directly integrated in the radiator, in the ground plane, or near the feeding line, to reduce the complexity of the electronic circuitry and the cost of the whole UWB communication system. Specifically they consist of suitable resonators that are strongly coupled (at the resonance frequencies) with the electromagnetic field excited in their neighborhood, thereby causing a destructive field emission. Given their interest in practical applications, the main filtering techniques adopted in the antenna design are discussed in this section.

Spade-shaped antenna for UWB applications with triple notched bands at 3.3-3.9, 5.2-5.35, and 5.8-6.0 GHz, realized to avoid interference with communication systems sharing the same operative environment, has been proposed in [62]. The triple notched frequency bands are achieved by employing a hook-shaped defected ground structure (DGS) in each side of the ground plane, embedding an $\Omega$-shaped slot on the radiating patch and adding a semi-octagon-shaped resonant ring on the backside of the antenna (see Figure 53). The analysis and design have been performed using the fullwave FEM-based HFSS [8]. The antenna is realized on a substrate of dimensions $36 \mathrm{~mm} \times 34 \mathrm{~mm} \times 1 \mathrm{~mm}$ having a dielectric relative permittivity of 2.65 . The average value of the gain is about $6 \mathrm{dBi}$, while the group delay is quite constant with the exception of the bands in which the antenna does not radiate.

A UWB planar slot antenna with dual-band-notched characteristics, useful to reduce interference with WiMAX and WLAN systems working in the same environment, has been presented in [64]. The basic antenna, realized on a FR4 dielectric substrate (dimensions $22 \mathrm{~mm} \times 24 \mathrm{~mm}$ ) with relative permittivity of 4.6 , thickness $1.6 \mathrm{~mm}$, and loss tangent 0.02 , consists of a house-shaped slot excited by means of a rectangular monopole connected to a $50 \Omega$ microstrip transmission line (see Figure 54).

To filter out the WiMAX band, an angle-shaped parasitic slit is asymmetrically etched out along with the rectangular monopole, while two symmetrical parasitic slits are placed inside the tapered slot to create another notched band for the WLAN frequency band. In particular, the parasitic slits introduce additional paths to the surface currents whose directions at the slits' resonant frequencies are opposite to those excited in the metal frame forming the slot antenna, thus reducing the energy emission. The antenna radiation pattern is omnidirectional in the $H$-plane, while in the $E$ plane two nulls similar to those of typical monopole antennas are present. The antenna exhibits a gain ranging between 2 and $5 \mathrm{dBi}$ and a fairly stable group delay in the whole UWB band except at the notched frequency bands. The full-wave MoM-based IE3D [87] has been employed to analyze and design the antenna.

A flexible UWB antenna for wearable applications, with a notched band useful to prevent interference with WLAN communication systems, was presented in [65]. The antenna, excited by means of a CPW, is realized on an ultra-thin liquid crystal polymer (LCP) substrate with relative permittivity of 2.9 and thickness of $0.05 \mathrm{~mm}$ on which a copper layer of only $0.018 \mathrm{~mm}$ is placed to realize the radiating element. Thanks to the resistance of the LCP substrate to bending (up to about $60^{\circ}$ ), to high temperatures, and to humidity, it can be employed by firefighters, sailors, and lifeguards. The antenna (size $26 \mathrm{~mm} \times 16 \mathrm{~mm} \times 0.068 \mathrm{~mm}$ ), whose geometry is shown 


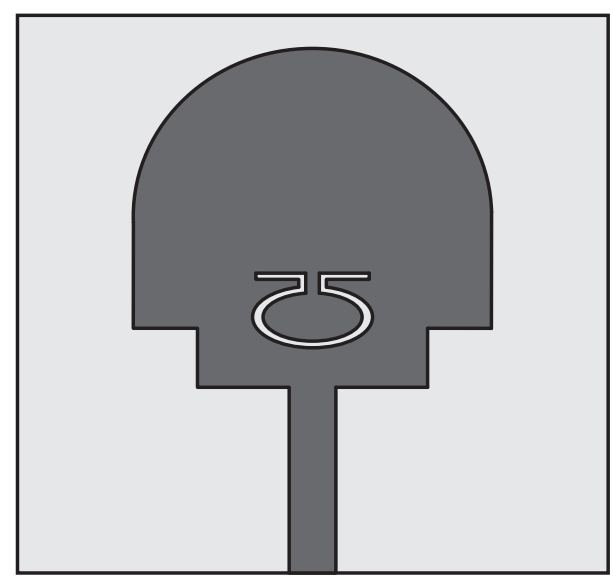

(a)

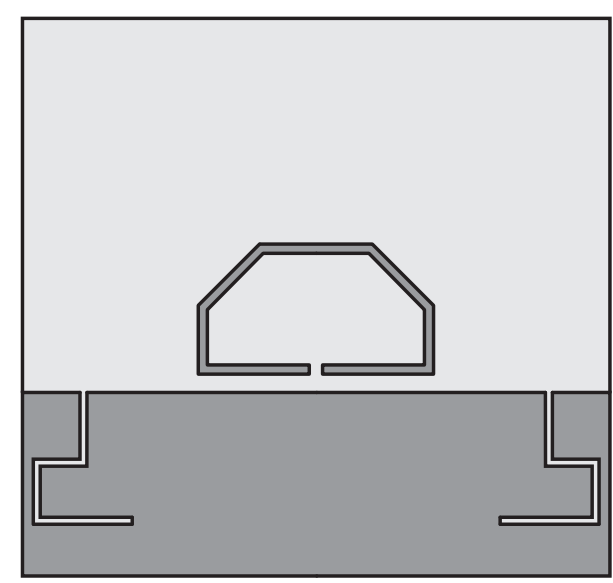

(b)

FIGURE 53: Geometry of a planar UWB monopole antenna with triple band-notched characteristics [62]. Top view (a) and bottom view (b). The resonant filters are shown in the figure. Dark gray upper metal face, medium gray bottom metal face, and light gray dielectric substrate.

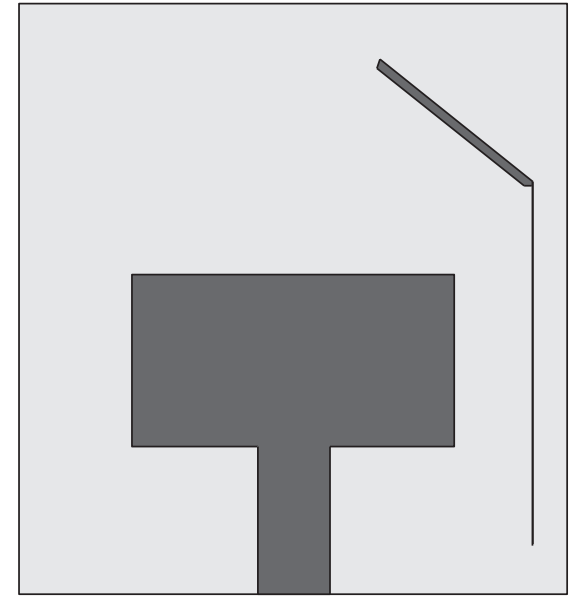

(a)

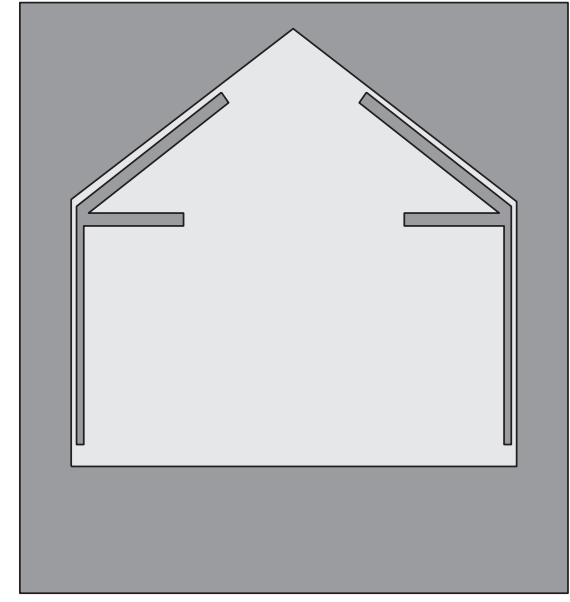

(b)

Figure 54: Geometry of the dual-band-notched UWB slot antenna [64]. (a) Front view. (b) Back view. The slits adopted to realize bandnotched characteristics are indicated. Dark gray upper metal face, medium gray bottom metal face, and light gray dielectric substrate.

in Figure 55, has two tapered slots defined by arc of ellipses to get a better impedance bandwidth.

The frequency notch is obtained by using an $\mathrm{H}$-shaped slot with equal arms and a small gap between them (see Figure 55). The H-shaped slot is placed near the feeding point so as to reflect the RF energy toward the feeding line when the slot resonates (at about $5.35 \mathrm{GHz}$ ). The structure has been analyzed and designed using the full-wave FITbased CST Microwave Studio [25]. The radiation patterns are nearly omnidirectional over the entire frequency range in the $H$-plane, while in the $E$-plane they are similar to that of a dipole up to about $3 \mathrm{GHz}$, while some fluctuations appear at higher frequencies where the antenna becomes more directive. The antenna has a gain of about 2-3 dBi and exhibits good time-domain characteristics, with measured fidelity factors ranging from 0.86 to 0.91 .

A compact low-profile antenna (see Figure 56), suitable to cover the UWB band (from 2.98 up to $10.76 \mathrm{GHz}$ ), consisting of a rectangular monopole, below which a triarms resonator, suitable to realize two notched bands around the frequencies of 3.5 (WiMAX) and $5.5 \mathrm{GHz}$ (WLAN), is placed, has been presented in [66]. The rectangular monopole, of size of $14.5 \mathrm{~mm} \times 14.75 \mathrm{~mm}$, is printed on a FR4 substrate having a permittivity of 4.4 and loss tangent 0.02 . The overall antenna size is of $29 \mathrm{~mm} \times 20.5 \mathrm{~mm}$. The two notched bands are realized by means of a triarm filter that, around the frequencies of 3.5 and $5.5 \mathrm{GHz}$, is excited by strong currents having opposite directions with respect to those excited on the ground plane. At the same time the strong electromagnetic coupling with the rectangular monopole produces a substantial reduction of the current amplitude excited on the metal patch with a consequent reduction of the antenna emission process.

The antenna, which exhibits omnidirectional radiation characteristics in the horizontal plane and monopole-type characteristics in vertical plane, presents a gain ranging from about 1.5 up to $5.9 \mathrm{dBi}$ and a group delay less than $2 \mathrm{~ns}$ 


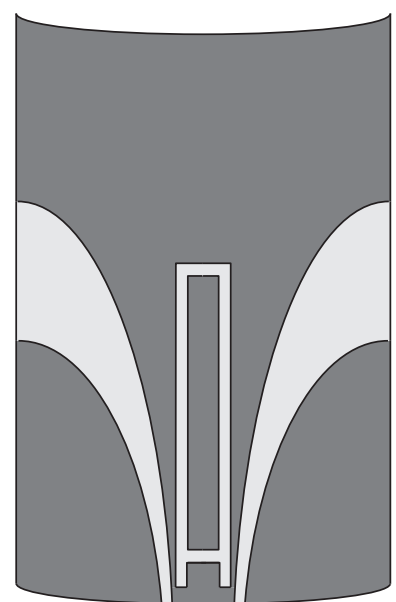

FIGURE 55: Geometry of the flexible band-notched UWB antenna [65]. The H-shaped slot adopted to realize the band-notched characteristics is indicated. Dark gray metal face and light gray dielectric substrate.

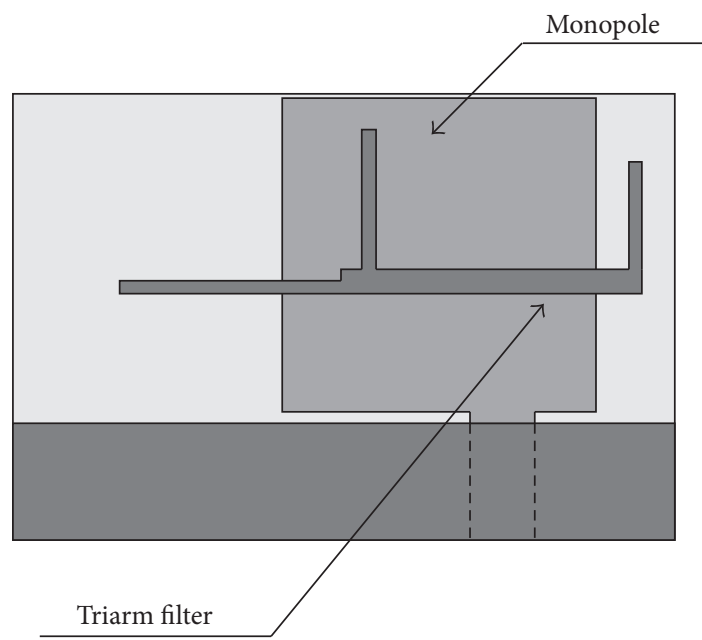

FIGURE 56: Geometry of a compact low-profile UWB antenna equipped with a triarms resonator [66]. Dark gray upper metal face, medium gray bottom metal face, and light gray dielectric substrate.

throughout the operating band, except in the notched bands, making the antenna suitable to support UWB pulse signals.

An example of a UWB rectangular dielectric resonator antenna (DRA) with bandstop performance has been presented in [67]. The antenna, whose geometry is shown in Figure 57, consists of a rectangular resonator of height $19 \mathrm{~mm}$, made of Rogers 6010 with a dielectric permittivity of 10.2 and a loss tangent of 0.003 , placed on a metallic ground of size of $90 \mathrm{~mm} \times 90 \mathrm{~mm}$. An air gap is placed under the DR to extend the antenna impedance bandwidth, while a metallic patch is used to excite the DR. To realize the 5.1-6 GHz notched band, two short-circuited metal strips are symmetrically attached on the opposite face of the DR, while a slit is realized on the metallic patch used to excite the DR. When both structures resonate, a marked impedance mismatch occurs between the feeding line and the antenna causing a frequency notch. The

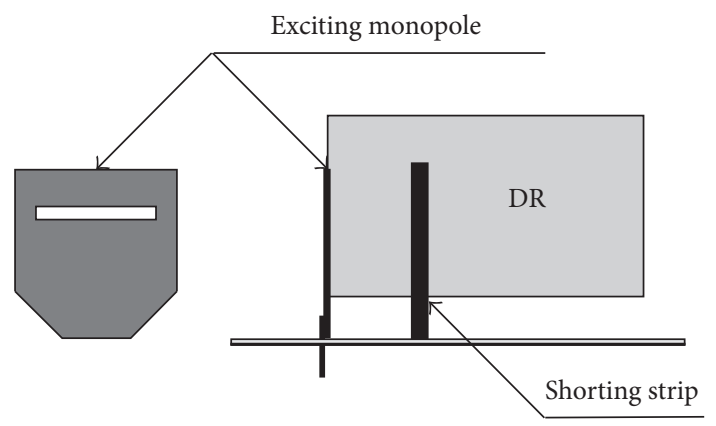

FIGURE 57: Geometry of a UWB rectangular DRA with a bandstop [67]. Dark gray metal and medium gray DR.

radiation patterns are symmetrical in the $H$-plane, while they are less symmetrical in the $E$-plane due to the excitation of the higher order modes. Finally, the antenna gain ranges between 6 and $9 \mathrm{dBi}$ in the UWB frequency band. The structure has been analyzed and designed using the full-wave FIT-based CST Microwave Studio [25].

Two different design solutions were introduced in [63] with the aim of enhancing the broadside gain of a printed monopole antenna equipped with an elliptical metallic radiator and with a notch inserted on the top edge of a cornerrounded ground plane. Specifically, in the first prototype, an arc-shaped slot was inserted in the patch, near the microstrip line, for broadside gain enhancement in the $E$-plane at the higher frequencies of the UWB band. The second antenna (see Figure 58) has the same geometry of the first antenna but with an additional single-wing filter for a further gain enhancement. Both antennas have impedance bandwidth ranging from 3 up to $11 \mathrm{GHz}$. The antenna, realized on a Rogers 4350B dielectric substrate of thickness $1.524 \mathrm{~mm}$, dielectric permittivity $\varepsilon_{r}=3.48$, and $\tan \delta=0.0038$, has dimensions of $25 \mathrm{~mm} \times 37 \mathrm{~mm}$. The full-wave FEM-based HFSS [8] has been adopted to perform the analysis and design of the mentioned antennas. The measured antenna gain ranges between 0 and $3 \mathrm{dBi}$, for the first antenna, and between 1 and $4 \mathrm{dBi}$, for the second antenna. Higher cross-polarization levels are observed in the second radiating structure due to horizontal currents excited on the multimode resonant filter (see Figure 58). Given the particular characteristics exhibited by these antennas, they can be used for highaccuracy positioning systems, portable devices, and cognitive radios.

A compact reconfigurable (bandstop/bandpass) and frequency-tunable radiating structure based on S-shaped split-ring resonators (S-SRRs) whose geometry is shown in Figure 59 has been proposed in [68]. This design solution can be usefully employed so as to avoid interference when other communication systems are installed in the same environment. The antenna, consisting in a standard UWB elliptical monopole excited by a $50 \Omega \mathrm{CPW}$, was realized on a Rogers Duroid 5880 dielectric material with relative permittivity 2.2, thickness $0.257 \mathrm{~mm}$, and loss tangent 0.0009 . A suitable filter, consisting of a Skyworks [204] SMV1405 varactor (capacitance range extending from 0.63 to $2.67 \mathrm{pF}$ ) 


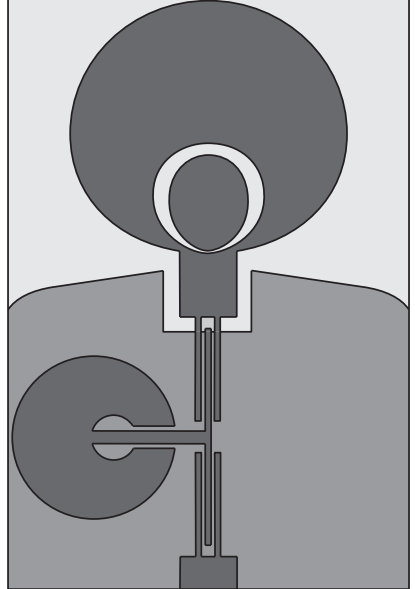

FIGURE 58: Printed monopole UWB antenna with an arc-shaped slot inserted in the patch and with a single-wing filter element employed to enhance the broadside antenna gain [63]. The multimode resonant filter is responsible for higher levels of cross-polarized field. Dark gray upper metal face, medium gray bottom metal face, and light gray dielectric substrate.

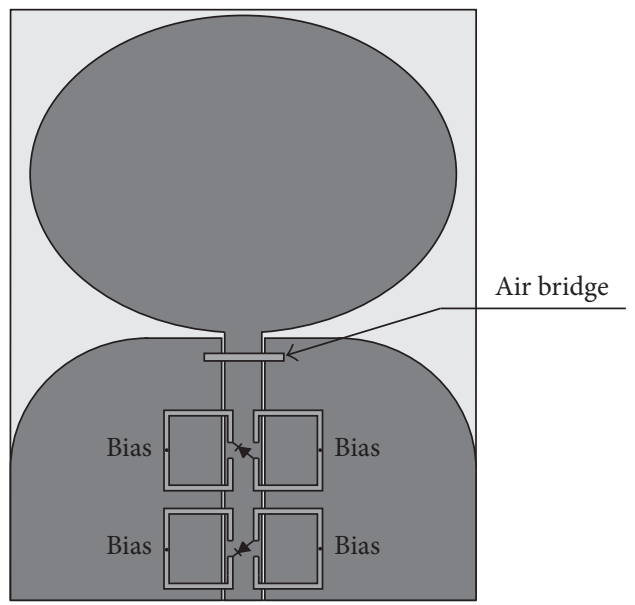

FIGURE 59: Geometry of the reconfigurable and tunable S-shaped split-ring resonators (S-SRRs) antenna for UWB applications [68]. An air bridge is adopted to suppress the slot line mode, which can be excited by the asymmetric structures forming the filter. Dark gray upper metal face, medium gray bottom split-ring resonator and air bridge, and light gray dielectric substrate.

placed at the center of an S-SRR, is integrated on the CPW line (where the magnetic field exhibits its maximum value) so as to realize a tunable bandstop frequency. Furthermore, the addition of two MACOM's [205] MADP-042305-130600 pin diodes arranged between the line and the ground plane acting as a pair of shunt inductors allows switching the behavior of the filter from bandstop to a bandpass characteristic. This makes it possible to realize a UWB antenna suitable to limit the electromagnetic interference with the WiMAX or WLAN systems. Experimental measurements performed on an antenna prototype confirm the numerical results carried out using the commercial full-wave FEM-based HFSS [8]. In addition, they also highlight the low impact that the filter integrated on the CPW has on the antenna radiation patterns with respect to those design solutions making use of filters directly integrated in the radiating element.

Additional information concerning antennas having characteristics similar to those presented in this section can be found in $[4,58-61,80,81,206-212]$.

\section{Conclusions}

A comprehensive review concerning broadband, multiband, and UWB antennas for mobile and wireless communication systems has been carried out. Geometries, materials, design solutions, and numerical tools adopted for the analysis and design have been investigated. Several classes of antennas useful for the above-mentioned applications have been identified. The electromagnetic performances, the dimensions, and the materials, as well as the design solutions useful to improve the antenna bandwidth of monopole (2D and $3 \mathrm{D}$ profile), printed dipole, metamaterial, wearable, and dielectric resonator antennas, have been taken into account.

Starting from the investigation carried out in this review paper, the following general considerations concerning the presented antennas can be highlighted.

(1) Monopole antennas placed over a ground plane emit energy in the spatial region located between the bottom edge of the monopole and the ground plane; therefore special attention should be paid to the shape of the monopole in this region. The emission process produces substantially radiation patterns with antenna beams oriented in the horizontal plane. Multipoint excitations (see, e.g., the trident-shaped feeding networks) tend to improve the operating band, the polarization purity, and the signal integrity of the radiated field useful in UWB applications. The ground plane makes it possible to limit interference with the sensitive electronic circuitry typically housed in the antenna back region. They are of simple realization and avoiding the use of dielectric materials does not exhibit the adverse effects caused by surface waves. The inherent $3 \mathrm{D}$ geometry limits their use to applications concerning base stations, automotive or avionics systems for high-speed vehicles.

(2) Printed monopoles exhibit a low-profile that allows their use in both fixed (base stations, automotive systems, etc.) and mobile terminals (smartphones, tablets, glasses, laptops, wearable computers, etc.), as well as a simple integration with the RF circuits. The use of dielectric materials implies the presence of surface waves; therefore the use of thin dielectric materials having low dielectric permittivities is strongly recommended. For these applications it might be useful to limit the interference with WLAN and WiMAX systems that may be present in the same environment. To this end, several filtering systems (simple or tunable) integrated on the monopole, on the ground plane or on the feeding line, can be adopted. In both cases it should be noticed that said 
filters are responsible for an alteration of the group delay, which can result in a significant distortion of the radiated signal with the appearance of a pronounced ringing. This process can be even more evident when use is made of metamaterials, which employ resonant structures to achieve special electromagnetic performances. In that case, a careful time-domain analysis of the radiated signal has to be performed if the antenna has to work also with pulse signals. To overcome these drawbacks additional research activities should be performed to find new metamaterial geometries suitable to guarantee stable group delay and limited ringing effects.

(3) Wearable antennas present additional design requirements because the user's body should be shielded as much as possible from the electromagnetic field generated by the antenna. In fact, the interaction gives rise to an energy deposition in the user's body resulting in decreased efficiency and alteration of the antenna radiation patterns. A design solution to this problem is the use of radiant structures making use of full ground. Anyway, for these antennas, the design must take into account of the user, using human body models during the numerical simulations (see, e.g., that based on the "visible human") as well as during the experimental measurements (anthropomorphic dummies). Moreover, for said antennas, the development of dedicated transitions, connectors, and flexible feeding lines to be easily integrated with the e-textiles would be desirable.

(4) Dielectric resonator antennas exhibiting 3D profile can be used in radio base stations or terrestrial and satellite radio links when a high energy efficiency is required, as they are not affected by metal losses or surface waves. The use of low permittivity dielectric materials and special geometries can ensure broadband behavior, while the choice of suitable excitation systems can guarantee excellent performance as concerns the linear/circular polarization. Moreover, metal reflectors and/or dielectric lenses can ensure higher gains over wide frequency ranges.

The analysis of the scientific literature has shown a remarkable technological readiness level reached by the $2 \mathrm{D}$ and 3D printed antenna geometries, while there are still ongoing research activities aimed to find new geometries and materials for advanced applications of dielectric resonator and wearable antennas. The full-wave commercial tools required for analysis and design of the considered antennas are reliable and user friendly. Even though further efforts should be made to develop appropriate numerical tools suitable to model more accurately the characteristics of the materials (roughness of simple and twisted fabrics) used to realize wearable antennas. As regards the substrates necessary for the realization of printed antennas, there is a large class of materials having high performances and low costs, while the dielectric materials useful for the realization of wearable and DRA antennas are still quite expensive. For these antennas the high material costs are the main causes that limit their broad spread in the market. New generations of high performance antennas will be developed in the near future thanks to the advent of $3 \mathrm{D}$ printers having high spatial resolution.

\section{Competing Interests}

The authors declare that there is no conflict of interests regarding the publication of this paper.

\section{References}

[1] K. W. Leung, E. H. Lim, and X. S. Fang, "Dielectric resonator antennas: from the basic to the aesthetic," Proceedings of the IEEE, vol. 100, no. 7, pp. 2181-2193, 2012.

[2] Y. Liu, L. Si, M. Wei et al., "Some recent developments of microstrip antenna," International Journal of Antennas and Propagation, vol. 2012, Article ID 428284, 10 pages, 2012.

[3] A. T. Mobashsher and A. Abbosh, "Utilizing symmetry of planar ultra-wideband antennas for size reduction and enhanced performance," IEEE Antennas and Propagation Magazine, vol. 57, no. 2, pp. 153-166, 2015.

[4] R. Cicchetti, A. Faraone, D. Caratelli, and M. Simeoni, "Wideband, multiband, tunable, and smart antenna systems for mobile and UWB wireless applications 2014," International Journal of Antennas and Propagation, vol. 2015, Article ID 536031, 3 pages, 2015.

[5] https://transition.fcc.gov/Bureaus/Engineering_Technology/ Orders/2002/fcc02048.pdf.

[6] G. A. Deschamps, "Microstrip microwave antennas," in Proceedings of the 3rd USAF Symposium on Antennas, October 1953.

[7] Y. Zhang, J. Liu, Z. Liang, and Y. Long, "A wide-bandwidth monopolar patch antenna with dual-ring couplers," International Journal of Antennas and Propagation, vol. 2014, Article ID 980120, 6 pages, 2014.

[8] ANSYS HFSS, http://www.ansys.com/Products/Electronics/ ANSYS-HFSS.

[9] D. Caratelli, R. Cicchetti, G. Bit-Babik, and A. Faraone, "A perturbed E-shaped patch antenna for wideband WLAN applications," IEEE Transactions on Antennas and Propagation, vol. 54, no. 6, pp. 1871-1874, 2006.

[10] D. Caratelli and R. Cicchetti, "A full-wave analysis of interdigital capacitors for planar integrated circuits," IEEE Transactions on Magnetics, vol. 39, no. 3, pp. 1598-1601, 2003.

[11] D. Caratelli, R. Cicchetti, G. Bit-Babik, and A. Faraone, "Circuit model and near-field behavior of a novel patch antenna for WWLAN applications," Microwave and Optical Technology Letters, vol. 49, no. 1, pp. 97-100, 2007.

[12] K. Mandal and P. P. Sarkar, "High gain wide-band u-shaped patch antennas with modified ground planes," IEEE Transactions on Antennas and Propagation, vol. 61, no. 4, pp. 2279-2282, 2013.

[13] A. Agrawal, P. K. Singhal, and A. Jain, "Design and optimization of a microstrip patch antenna for increased bandwidth," International Journal of Microwave and Wireless Technologies, vol. 5, no. 4, pp. 529-535, 2013.

[14] S. K. Pavuluri, C. Wang, and A. J. Sangster, "High efficiency wideband aperture-coupled stacked patch antennas assembled using millimeter thick micromachined polymer structures," IEEE Transactions on Antennas and Propagation, vol. 58, no. 11, pp. 3616-3621, 2010. 
[15] J. M. Kovitz and Y. Rahmat-Samii, "Using thick substrates and capacitive probe compensation to enhance the bandwidth of traditional CP patch antennas," IEEE Transactions on Antennas and Propagation, vol. 62, no. 10, pp. 4970-4979, 2014.

[16] K. Noguchi, H. Rajagopalan, and Y. Rahmat-Samii, "Design of wideband/dual-band E-shaped patch antennas with the transmission line mode theory," IEEE Transactions on Antennas and Propagation, vol. 64, no. 4, pp. 1183-1192, 2016.

[17] G. Dubost and S. Zisler, Antennas a Large Band, Masson, New York, NY, USA, 1976.

[18] R. Cicchetti, O. Testa, and D. Caratelli, "A numerical procedure for the analysis of EMC/EMI problems in radio communication systems operating in complex environments," IEEE Transactions on Electromagnetic Compatibility, vol. 54, no. 6, pp. 12691280, 2012.

[19] D. Valderas, B. Sedano, A. Garcia-Alonso, and J. I. Sancho, "Synthesis of TLM-based UWB planar monopole impedance bandwidth," IEEE Transactions on Antennas and Propagation, vol. 55, no. 10, pp. 2874-2879, 2007.

[20] D. Valderas, J. Legarda, I. Gutiérrez, and J. I. Sancho, "Design of UWB folded-plate monopole antennas based on TLM," IEEE Transactions on Antennas and Propagation, vol. 54, no. 6, pp. 1676-1687, 2006.

[21] M. J. Ammann and Z. N. Chen, "Wideband monopole antennas for multi-band wireless systems," IEEE Antennas and Propagation Magazine, vol. 45, no. 2, pp. 146-150, 2003.

[22] M. J. Ammann, "Control of the impedance bandwidth of wideband planar monopole antennas using a beveling technique," Microwave and Optical Technology Letters, vol. 30, no. 4, pp. 229-232, 2001.

[23] S.-Y. Suh, W. L. Stutzman, and W. A. Davis, "A new ultrawideband printed monopole antenna: the planar Inverted Cone Antenna (PICA)," IEEE Transactions on Antennas and Propagation, vol. 52, no. 5, pp. 1361-1365, 2004.

[24] X.-F. Bai, S.-S. Zhong, and X.-L. Liang, "Leaf-shaped monopole antenna with extremely wide bandwidth," Microwave and Optical Technology Letters, vol. 48, no. 7, pp. 1247-1250, 2006.

[25] CST Microwave Studio, https://www.cst.com/.

[26] Y. Chen, S. Yang, S. He, and Z. Nie, "Design and analysis of wideband planar monopole antennas using the multilevel fast multipole algorithm," Progress In Electromagnetics Research B, no. 15, pp. 95-112, 2009.

[27] M. J. Ammann and Z. N. Chen, "A wide-band shorted planar monopole with bevel," IEEE Transactions on Antennas and Propagation, vol. 51, no. 4, pp. 901-903, 2003.

[28] K.-L. Wong, C.-H. Wu, and S.-W. Su, "Ultrawide-band square planar metal-plate monopole antenna with a trident-shaped feeding strip," IEEE Transactions on Antennas and Propagation, vol. 53, no. 4, pp. 1262-1269, 2005.

[29] The IEEE 802.16 Working Group on Broadband Wireless Access Standards, http://grouper.ieee.org/groups/802/16/index.html.

[30] S. Ghosh, "Design of planar crossed monopole antenna for ultrawideband communication," IEEE Antennas and Wireless Propagation Letters, vol. 10, pp. 548-551, 2011.

[31] WIPL-D Pro. Information, http://www.wipl-d.com/.

[32] W.-S. Lee, K.-S. Oh, and J.-W. Yu, "A wideband planar monopole antenna array with circular polarized and band-notched characteristics," Progress in Electromagnetics Research, vol. 128, pp. 381-398, 2012.

[33] B. Chen, Y.-C. Jiao, F.-C. Ren, and L. Zhang, "Broadband monopole antenna with wideband circular polarization,"
Progress in Electromagnetics Research Letters, vol. 32, pp. 19-28, 2012.

[34] A. Singh and S. Singh, "A novel CPW-fed wideband printed monopole antenna with DGS," AEU-International Journal of Electronics and Communications, vol. 69, no. 1, pp. 299-306, 2015.

[35] S.-S. Zhong, X.-L. Liang, and W. Wang, "Compact elliptical monopole antenna with impedance bandwidth in excess of 21:1," IEEE Transactions on Antennas and Propagation, vol. 55, no. 11, pp. 3082-3085, 2007.

[36] J. Wu, J. Ke, C. Jou, and C. Wang, "Microstrip-fed broadband circularly polarised monopole antenna," IET Microwaves, Antennas and Propagation, vol. 4, no. 4, pp. 518-525, 2010.

[37] K. H. Chiang and K. W. Tam, "Microstrip monopole antenna with enhanced bandwidth using defected ground structure," IEEE Antennas and Wireless Propagation Letters, vol. 7, pp. 532 $535,2008$.

[38] K. Ding, C. Gao, T. Yu, and D. Qu, "Broadband C-shaped circularly polarized monopole antenna," IEEE Transactions on Antennas and Propagation, vol. 63, no. 2, pp. 785-790, 2015.

[39] M. A. Antoniades and G. V. Eleftheriades, "A compact multiband monopole antenna with a defected ground plane," IEEE Antennas and Wireless Propagation Letters, vol. 7, pp. 652-655, 2008.

[40] Y. Li and W. Yu, "A miniaturized triple band monopole antenna for WLAN and WiMAX applications," International Journal of Antennas and Propagation, vol. 2015, Article ID 146780, 5 pages, 2015.

[41] S. Verma and P. Kumar, "Compact arc-shaped antenna with binomial curved conductor-backed plane for multiband wireless applications," IET Microwaves, Antennas and Propagation, vol. 9, no. 4, pp. 351-359, 2015.

[42] W. J. Krzysztofik, "Modified Sierpinski fractal monopole for ISM-bands handset applications," IEEE Transactions on Antennas and Propagation, vol. 57, no. 3, pp. 606-615, 2009.

[43] M. A. Antoniades and G. V. Eleftheriades, "A multiband monopole antenna with an embedded reactance-cancelling transmission-line matching network," IEEE Antennas and Wireless Propagation Letters, vol. 9, pp. 1107-1110, 2010.

[44] M. G. N. Alsath and M. Kanagasabai, "A shared-aperture multiservice antenna for automotive communications," IEEE Antennas and Wireless Propagation Letters, vol. 13, pp. 14171420, 2014.

[45] N. A. Abbasi and R. J. Langley, "Multiband-integrated antenna/ artificial magnetic conductor," IET Microwaves, Antennas and Propagation, vol. 5, no. 6, pp. 711-717, 2011.

[46] H. F. Abutarboush and A. Shamim, "Wide frequency independently controlled dual-band inkjet-printed antenna," IET Microwaves, Antennas and Propagation, vol. 8, no. 1, pp. 52-56, 2014.

[47] Y. Yao, X. Wang, and J. Yu, "Multiband planar monopole antenna for LTE MIMO systems," International Journal of Antennas and Propagation, vol. 2012, Article ID 890705, 6 pages, 2012.

[48] F. Paredes, G. Zamora, S. Zufanelli, F. J. Herraiz-Martínez, J. Bonache, and F. Martín, "Recent advances in multiband printed antennas based on metamaterial loading," Advances in OptoElectronics, vol. 2012, Article ID 968780, 12 pages, 2012.

[49] D. R. Melo, M. N. Kawakatsu, D. C. Nascimento, and V. Dmitriev, "A planar monopole UWB antennas with rounded patch and ground plane possessing improved impedance matching," Microwave and Optical Technology Letters, vol. 54, no. 2, pp. 335-338, 2012. 
[50] S. H. Choi, J. K. Park, S. K. Kim, and J. Y. Park, "A new ultra-wideband antenna for UWB applications," Microwave and Optical Technology Letters, vol. 40, no. 5, pp. 399-401, 2004.

[51] S. Verma and P. Kumar, "Printed Newton's egg curved monopole antenna for ultrawideband applications," IET Microwaves, Antennas and Propagation, vol. 8, no. 4, pp. 278-286, 2014.

[52] J. Liu, K. P. Esselle, S. G. Hay, and S. Zhong, "Effects of printed UWB antenna miniaturization on pulse fidelity and pattern stability," IEEE Transactions on Antennas and Propagation, vol. 62, no. 8, pp. 3903-3910, 2014.

[53] L. Ge and K. M. Luk, "A magneto-electric dipole for unidirectional UWB communications," IEEE Transactions on Antennas and Propagation, vol. 61, no. 11, pp. 5762-5765, 2013.

[54] A. T. Mobashsher and A. Abbosh, "Three-dimensional folded antenna with ultra-wideband performance, directional radiation and compact size," IET Microwaves, Antennas and Propagation, vol. 8, no. 3, pp. 171-179, 2014.

[55] G. S. Reddy, A. Kamma, S. Kharche, J. Mukherjee, and S. K. Mishra, "Cross-configured directional UWB antennas for multidirectional pattern diversity characteristics," IEEE Transactions on Antennas and Propagation, vol. 63, no. 2, pp. 853-858, 2015.

[56] A. Elsherbini, J. Wu, and K. Sarabandi, "Dual polarized wideband directional coupled sectorial loop antennas for radar and mobile base-station applications," IEEE Transactions on Antennas and Propagation, vol. 63, no. 4, pp. 1505-1513, 2015.

[57] A. T. Mobashsher and A. M. Abbosh, "Performance of directional and omnidirectional antennas in wideband head imaging," IEEE Antennas and Wireless Propagation Letters, vol. 15, pp. 1618-1621, 2016.

[58] Y. Zhu, F. S. Zhang, C. Lin, Q. Zhang, and J. X. Huang, "A novel dual band-notched monopole antenna for ultrawideband application," Progress In Electromagnetics Research Letters, vol. 16, pp. 109-117, 2010.

[59] R. Eshtiaghi, R. Zaker, J. Nouronia, and C. Ghobadi, "UWB semi-elliptical printed monopole antenna with subband rejection filter," AEU-International Journal of Electronics and Communications, vol. 64, no. 2, pp. 133-141, 2010.

[60] D. Dong, S. Chen, Z. Liao, and G. Liu, "A CPW-fed dual-bandnotched antenna with sharp skirt selectivity for UWB applications," International Journal of Antennas and Propagation, vol. 2014, Article ID 629387, 7 pages, 2014.

[61] D. Draskovic, J. R. O. Fernandez, and C. Briso Rodríguez, "Planar ultrawideband antenna with photonically controlled notched bands," International Journal of Antennas and Propagation, vol. 2013, Article ID 924768, 6 pages, 2013.

[62] W. T. Li, X. W. Shi, and Y. Q. Hei, "Novel planar UWB monopole antenna with triple band-notched characteristics," IEEE Antennas and Wireless Propagation Letters, vol. 8, pp. 1094-1098, 2009.

[63] M.-C. Tang, T. Shi, and R. W. Ziolkowski, "Planar ultrawideband antennas with improved realized gain performance," IEEE Transactions on Antennas and Propagation, vol. 64, no. 1, pp. 6169, 2016.

[64] R. Azim, M. T. Islam, and A. T. Mobashsher, "Design of a dual band-notch UWB slot antenna by means of simple parasitic slits," IEEE Antennas and Wireless Propagation Letters, vol. 12, pp. 1412-1415, 2013.

[65] Q. H. Abbasi, M. U. Rehman, X. Yang, A. Alomainy, K. Qaraqe, and E. Serpedin, "Ultrawideband band-notched flexible antenna for wearable applications," IEEE Antennas and Wireless Propagation Letters, vol. 12, no. 417, pp. 1606-1609, 2013.
[66] R. Azim, M. T. Islam, and A. T. Mobashsher, "Dual band-notch UWB antenna with single tri-arm resonator," IEEE Antennas and Wireless Propagation Letters, vol. 13, pp. 670-673, 2014.

[67] Y. F. Wang, T. A. Denidni, Q. S. Zeng, and G. Wei, "Bandnotched UWB rectangular dielectric resonator antenna," Electronics Letters, vol. 50, no. 7, pp. 483-484, 2014.

[68] A. K. Horestani, Z. Shaterian, J. Naqui, F. Martín, and C. Fumeaux, "Reconfigurable and tunable S-shaped split-ring resonators and application in band-notched UWB antennas," IEEE Transactions on Antennas and Propagation, vol. 64, no. 9, pp. 3766-3776, 2016.

[69] A. Ghobadi and M. Dehmollaian, "A printed circularly polarized Y-shaped monopole antenna," IEEE Antennas and Wireless Propagation Letters, vol. 11, pp. 22-25, 2012.

[70] Y.-M. Cai, K. Li, Y.-Z. Yin, and W. Hu, "Broadband circularly polarized printed antenna with branched microstrip feed," IEEE Antennas and Wireless Propagation Letters, vol. 13, pp. 674-677, 2014.

[71] J.-Y. Sze, Z.-H. Zhou, C.-I. G. Hsu, and C.-H. Lin, "Broadband circularly polarized printed monopole antenna with unidirectional radiation patterns," Microwave and Optical Technology Letters, vol. 56, no. 7, pp. 1535-1540, 2014.

[72] M. Koohestani, M. N. Moghadasi, and B. S. Virdee, "Miniature microstrip-fed ultra-wideband printed monopole antenna with a partial ground plane structure," IET Microwaves, Antennas and Propagation, vol. 5, no. 14, pp. 1683-1689, 2011.

[73] J. Yang and A. Kishk, "A novel low-profile compact directional ultra-wideband antenna: the self-grounded Bow-Tie antenna," IEEE Transactions on Antennas and Propagation, vol. 60, no. 3, pp. 1214-1220, 2012.

[74] J. Liu, S. Zhong, and K. P. Esselle, "A printed elliptical monopole antenna with modified feeding structure for bandwidth enhancement," IEEE Transactions on Antennas and Propagation, vol. 59, no. 2, pp. 667-670, 2011.

[75] R. Kumar and S. Gaikwad, "On the design of nano-arm fractal antenna for UWB wireless applications," Journal of Microwaves, Optoelectronics and Electromagnetic Applications, vol. 12, no. 1, pp. 158-172, 2013.

[76] R. Azim, M. T. Islam, and N. Misran, "Ground modified doublesided printed compact UWB antenna," Electronics Letters, vol. 47, no. 1, pp. 9-11, 2011.

[77] M. T. Islam, R. Azim, and A. T. Mobashsher, "Triple bandnotched planar UWB antenna using parasitic strips," Progress in Electromagnetics Research, vol. 129, pp. 161-179, 2012.

[78] A. Kumar and K. V. Machavaram, "Microstrip filter with defected ground structure: a close perspective," International Journal of Microwave and Wireless Technologies, vol. 5, no. 5, pp. 589-602, 2013.

[79] J. Liu, K. P. Esselle, S. G. Hay, and S. S. Zhong, "Study of an extremely wideband monopole antenna with triple bandnotched charactersistics," Progress in Electromagnetics Research, vol. 123, pp. 143-158, 2012.

[80] Y. Xiao, Z.-Y. Wang, J. Li, Z.-L. Yuan, and A. K. Qin, "Twostep beveled UWB printed monopole antenna with band notch," International Journal of Antennas and Propagation, vol. 2014, Article ID 173704, 11 pages, 2014.

[81] O. Ahmed and A.-R. Sebak, "A printed monopole antenna with two steps and a circular slot for UWB applications," IEEE Antennas and Wireless Propagation Letters, vol. 7, pp. 411-413, 2008. 
[82] V. Sipal, B. Allen, D. Edwards, and B. Honary, "Twenty years of ultrawideband: opportunities and challenges," IET Communications, vol. 6, no. 10, pp. 1147-1162, 2012.

[83] P. Bernardi, R. Cicchetti, and O. Testa, "An accurate UTD model for the analysis of complex indoor radio environments in microwave WLAN systems," IEEE Transactions on Antennas and Propagation, vol. 52, no. 6, pp. 1509-1520, 2004.

[84] W. S. Lee, S. Y. Cha, K. S. Oh, and J. W. Yu, "Wideband circularly polarised planar monopole antenna array," Electronics Letters, vol. 47, no. 25, pp. 1358-1360, 2011.

[85] B. Li, Y. Ding, and Y.-Z. Yin, "A novel dual-band circularly polarized rectangular slot antenna," International Journal of Antennas and Propagation, vol. 2016, Article ID 9071610, 8 pages, 2016.

[86] M. T. Islam and R. Azim, "Recent trends in printed ultrawideband (UWB) antennas," in Advancement in Microstrip Antennas with Recent Applications, A. Kishk, Ed., pp. 173-201, InTech, Rijeka, Croatia, 2013.

[87] IE3D Software. Information, https://www.mentor.com/pcb/xpedition/simulation.

[88] H.-W. Liu, C.-H. Ku, T.-S. Wang, and C.-F. Yang, "Compact monopole antenna with band-notched characteristic for UWB applications," IEEE Antennas and Wireless Propagation Letters, vol. 9, pp. 397-400, 2010.

[89] D. Guha, S. Biswas, and C. Kumar, "Printed antenna designs using defected ground structures: a review of fundamentals and state-of-the-art developments," in Proceedings of the Forum for Electromagnetic Research Methods and Application Technologies (FERMAT '14), vol. 2, pp. 1-13, April 2014.

[90] S. S. Tiang, M. S. Hathal, N. S. Nik Anwar, M. F. Ain, and M. Z. Abdullah, "Development of a compact wide-slot antenna for early stage breast cancer detection featuring circular array full-view geometry," International Journal of Antennas and Propagation, vol. 2014, Article ID 309321, 11 pages, 2014.

[91] R. Azim and M. T. Islam, "Printed wide slot ultra-wideband antenna," in Advancement in Microstrip Antennas with Recent Applications, A. Kishk, Ed., chapter 7, InTech, 2013.

[92] S. Zhong, X. Yan, and X. Liang, "UWB planar antenna technology," Frontiers of Electrical and Electronic Engineering in China, vol. 3, no. 2, pp. 136-144, 2008.

[93] X.-L. Liang, T. A. Denidni, L.-N. Zhang, R.-H. Jin, J.-P. Geng, and Q. Yu, "Printed binomial-curved slot antennas for various wideband applications," IEEE Transactions on Microwave Theory and Techniques, vol. 59, no. 4, pp. 1058-1065, 2011.

[94] T. Karacolak and E. Topsakal, "A double-sided rounded bow-tie antenna (DSRBA) for UWB communication," IEEE Antennas and Wireless Propagation Letters, vol. 5, no. 1, pp. 446-449, 2006.

[95] G. Cappelletti, D. Caratelli, R. Cicchetti, and M. Simeoni, "A low-profile printed drop-shaped dipole antenna for wideband wireless applications," IEEE Transactions on Antennas and Propagation, vol. 59, no. 10, pp. 3526-3535, 2011.

[96] G. Cappelletti, D. Caratelli, and R. Cicchetti, "A locally conformal FDTD analysis of a thin printed antenna for wideband wireless applications," International Journal of Numerical Modelling: Electronic Networks, Devices and Fields, vol. 24, no. 3, pp. 257270, 2011.

[97] P. Bernardi, R. Cicchetti, S. Pisa, E. Pittella, E. Piuzzi, and O. Testa, "Design, realization, and test of a UWB radar sensor for breath activity monitoring," IEEE Sensors Journal, vol. 14, no. 2, pp. 584-596, 2014.
[98] X. N. Low, Z. N. Chen, and T. S. P. See, "A UWB dipole antenna with enhanced impedance and gain performance," IEEE Transactions on Antennas and Propagation, vol. 57, no. 10, pp. 2959-2966, 2009.

[99] S. X. Ta and I. Park, "Cavity-backed angled-dipole antennas for millimeter-wave wireless applications," International Journal of Antennas and Propagation, vol. 2016, Article ID 5083807, 11 pages, 2016.

[100] K. Li, C. Zhu, L. Li, Y.-M. Cai, and C.-H. Liang, "Design of electrically small metamaterial antenna with ELC and EBG loading," IEEE Antennas and Wireless Propagation Letters, vol. 12, pp. 678-681, 2013.

[101] M. S. A. Rani, S. K. A. Rahim, M. R. Kamarudin, T. Peter, S. W. Cheung, and B. M. Saad, "Electromagnetic behaviors of thin film CPW-fed CSRR loaded on UWB transparent antenna," IEEE Antennas and Wireless Propagation Letters, vol. 13, pp. 1239-1242, 2014.

[102] M. M. Islam, M. T. Islam, M. Samsuzzaman, and M. R. I. Faruque, "Compact metamaterial antenna for UWB applications," Electronics Letters, vol. 51, no. 16, pp. 1222-1224, 2015.

[103] J. Zhong, Y. Huang, G. Wen, H. Sun, O. Gordon, and W. Zhu, "Dual-band negative permittivity metamaterial based on cross circular loop resonator with shorting stubs," IEEE Antennas and Wireless Propagation Letters, vol. 11, pp. 803-806, 2012.

[104] M. A. W. Nordin, M. T. Islam, and N. Misran, "Design of a compact ultrawideband metamaterial antenna based on splitring resonator and capacitively loaded strips unit cell," Progress In Electromagnetics Research, vol. 136, pp. 157-173, 2013.

[105] W. Liu, Z. N. Chen, and X. Qing, "60-GHz thin broadband high-gain LTCC metamaterial-mushroom antenna array," IEEE Transactions on Antennas and Propagation, vol. 62, no. 9, pp. 4592-4601, 2014.

[106] M. Alibakhshi-Kenari and M. Naser-Moghadasi, "Novel UWB miniaturized integrated antenna based on CRLH metamaterial transmission lines," AEU - International Journal of Electronics and Communications, vol. 69, no. 8, pp. 1143-1149, 2015.

[107] M. Alibakhshi-Kenari, M. Naser-Moghadasi, R. A. Sadeghzadeh, and B. S. Virdee, "Metamaterial-based antennas for integration in UWB transceivers and portable microwave handsets," International Journal of RF and Microwave Computer-Aided Engineering, vol. 26, no. 1, pp. 88-96, 2016.

[108] R. K. Saraswat and M. Kumar, "Miniaturized slotted ground UWB antenna loaded with metamaterial for WLAN and WiMax applications," Progress In Electromagnetics Research B, vol. 65, pp. 65-80, 2016.

[109] IEEE, "Wireless Medium Access Control (MAC) and Physical Layer (PHY) Specifications for Wireless Personal Area Networks (WPANs) used in or around a body," IEEE P802.15.6/D01, 2010.

[110] F. Declercq, I. Couckuyt, H. Rogier, and T. Dhaene, "Environmental high frequency characterization of fabrics based on a novel surrogate modelling antenna technique," IEEE Transactions on Antennas and Propagation, vol. 61, no. 10, pp. 5200-5213, 2013.

[111] R. Salvado, C. Loss, R. Gonçalves, and P. Pinho, "Textile materials for the design of wearable antennas: a survey," Sensors, vol. 12, no. 11, pp. 15841-15857, 2012.

[112] Y. Ouyang and W. J. Chappell, "High frequency properties of electro-textiles for wearable antenna applications," IEEE Transactions on Antennas and Propagation, vol. 56, no. 2, pp. 381-389, 2008. 
[113] N. Chahat, M. Zhadobov, S. A. Muhammad, L. Le Coq, and R. Sauleau, "60-GHz textile antenna array for body-centric communications," IEEE Transactions on Antennas and Propagation, vol. 61, no. 4, pp. 1816-1824, 2013.

[114] S. Sankaralingam and B. Gupta, "Determination of dielectric constant of fabric materials and their use as substrates for design and development of antennas for wearable applications," IEEE Transactions on Instrumentation and Measurement, vol. 59, no. 12, pp. 3122-3130, 2010.

[115] S. Lemey, F. Declercq, and H. Rogier, "Dual-band substrate integrated waveguide textile antenna with integrated solar harvester," IEEE Antennas and Wireless Propagation Letters, vol. 13, pp. 269-272, 2014.

[116] G. Monti, L. Corchia, and L. Tarricone, "UHF wearable rectenna on textile materials," IEEE Transactions on Antennas and Propagation, vol. 61, no. 7, pp. 3869-3873, 2013.

[117] M. Mantash, A.-C. Tarot, S. Collardey, and K. Mahdjoubi, "Investigation of flexible textile antennas and AMC reflectors," International Journal of Antennas and Propagation, vol. 2012, Article ID 236505, 10 pages, 2012.

[118] S. E. Morris, Y. Bayram, L. Zhang, Z. Wang, M. Shtein, and J. L. Volakis, "High-strength, metalized fibers for conformal load bearing antenna applications," IEEE Transactions on Antennas and Propagation, vol. 59, no. 9, pp. 3458-3462, 2011.

[119] A. Tsolis, W. G. Whittow, A. A. Alexandridis, and J. Y. C. Vardaxoglou, "Embroidery and related manufacturing techniques for wearable antennas: challenges and opportunities," Electronics, vol. 3, no. 2, pp. 314-338, 2014.

[120] B. Sanz-Izquierdo, J. C. Batchelor, and M. I. Sobhy, "Button antenna on textiles for wireless local area network on body applications," IET Microwaves, Antennas and Propagation, vol. 4, no. 11, pp. 1980-1987, 2010.

[121] B. Mandal and S. K. Parui, "Wearable tri-band SIW based antenna on leather substrate," Electronics Letters, vol. 51, no. 20, pp. 1563-1564, 2015.

[122] S. Zhu and R. Langley, "Dual-band wearable textile antenna on an EBG substrate," IEEE Transactions on Antennas and Propagation, vol. 57, no. 4, pp. 926-935, 2009.

[123] H. S. Zhang, S. L. Chai, K. Xiao, and L. F. Ye, "Numerical and experimental analysis of wideband E-shaped patch textile antenna," Progress In Electromagnetics Research C, vol. 45, pp. 163-178, 2013.

[124] M. A. R. Osman, M. K. A. Rahim, N. A. Samsuri, M. K. Elbasheer, and M. E. Ali, "Textile UWB antenna bending and wet performances," International Journal of Antennas and Propagation, vol. 2012, Article ID 251682, 12 pages, 2012.

[125] P. J. Soh, G. A. E. Vandenbosch, S. L. Ooi, and M. R. N. Husna, "Wearable dual-band Sierpinski fractal PIFA using conductive fabric," Electronics Letters, vol. 47, no. 6, pp. 365-367, 2011.

[126] M. E. Jalil, M. Kamal, A. Rahim, M. Azfar Abdullah, and O. Ayop, "Compact CPW-fed ultra-wideband (UWB) antenna using denim textile material," in Proceedings of the International Symposium on Antennas and Propagation (ISAP '12), pp. 30-33, Nagoya, Japan, October-November 2012.

[127] N. Lacerda Silva, L. M. Gonçalves, and H. Carvalho, "Deposition of conductive materials on textile and polymeric flexible substrates," Journal of Materials Science: Materials in Electronics, vol. 24, no. 2, pp. 635-643, 2013.

[128] H. A. Rahim, M. F. Abd Malek, I. Adam et al., "Effect of different substrate materials on a wearable textile monopole antenna," in Proceedings of the IEEE Symposium on Wireless Technology and
Applications (ISWTA '12), pp. 245-247, Bandung, Indonesia, September 2012.

[129] M. S. Shakhirul, M. Jusoh, A. Sahadah, C. M. Nor, and H. A. Rahim, "Embroidered wearable textile antenna on bending and wet performances for UWB reception," Microwave and Optical Technology Letters, vol. 56, no. 9, pp. 2158-2163, 2014.

[130] http://www.lairdtech.com/products/3055-233.

[131] D. H. Werner and Z. H. Jiang, Electromagnetics of Body-Area Networks: Antennas, Propagation, and RF Systems, John Wiley \& Sons, Hoboken, NJ, USA, 2016.

[132] http://www.statex.biz/images/pdf/woven/ShieldexNora_02.05.13 .pdf.

[133] Q. Bai, J. Rigelsford, and R. Langley, "Crumpling of microstrip antenna array," IEEE Transactions on Antennas and Propagation, vol. 61, no. 9, pp. 4567-4576, 2013.

[134] http://www.stockwell.com/data-sheets/3027217-nickel-polyesternonwoven.pdf.

[135] http://th-energy.hu/docs/Conductive\%20Metallized\%20Products_2.pdf.

[136] http://www.lessemf.com/fabric.html.

[137] M. I. Jais, M. F. Malek, M. F. Jamlos, and M. Jusoh, "Conductive E-textile analysis of $1.575 \mathrm{GHz}$ rectangular antenna with $\mathrm{H}$ slot for GPS application," in Proceedings of the Loughborough Antennas and Propagation Conference (LAPC '12), IEEE, Leicestershire, UK, November 2012.

[138] D. Baiya, On the development of conductive textile antennas [M.S. thesis], University of Tennessee, Knoxville, Tenn, USA, 2014, http://trace.tennessee.edu/utk_gradthes/3138.

[139] B. Ivsic, D. Bonefacic, and J. Bartolic, "Considerations on embroidered textile antennas for wearable applications," IEEE Antennas and Wireless Propagation Letters, vol.12, pp. 1708-1711, 2013.

[140] M. L. Scarpello, I. Kazani, C. Hertleer, H. Rogier, and D. V. Ginste, "Stability and efficiency of screen-printed wearable and washable antennas," IEEE Antennas and Wireless Propagation Letters, vol. 11, pp. 838-841, 2012.

[141] J. Lilja, V. Pynttäri, T. Kaija et al., "Body-worn antennas making a splash: lifejacket-integrated antennas for global search and rescue satellite system," IEEE Antennas and Propagation Magazine, vol. 55, no. 2, pp. 324-341, 2013.

[142] T. Kellomäki, J. Virkki, S. Merilampi, and L. Ukkonen, “Towards washable wearable antennas: a comparison of coating materials for screen-printed textile-based UHF RFID tags," International Journal of Antennas and Propagation, vol. 2012, Article ID 476570, 11 pages, 2012.

[143] L. Zhang, Z. Wang, and J. L. Volakis, “Textile antennas and sensors for body-worn applications," IEEE Antennas and Wireless Propagation Letters, vol. 11, pp. 1690-1693, 2012.

[144] P. B. Samal, P. J. Soh, and G. A. E. Vandenbosch, "UWB alltextile antenna with full ground plane for off-body WBAN communications," IEEE Transactions on Antennas and Propagation, vol. 62, no. 1, pp. 102-108, 2014.

[145] L. A. Yimdjo Poffelie, P. J. Soh, S. Yan, and G. A. Vandenbosch, "A high-fidelity all-textile UWB antenna with low back radiation for off-body WBAN applications," IEEE Transactions on Antennas and Propagation, vol. 64, no. 2, pp. 757-760, 2016.

[146] S. Lemey, T. Castel, P. Van Torre et al., "Threefold rotationally symmetric SIW antenna array for ultra-short-range MIMO communication," IEEE Transactions on Antennas and Propagation, vol. 64, no. 5, pp. 1689-1699, 2016. 
[147] P. B. Samal, P. J. Soh, and G. A. E. Vandenbosch, "A systematic design procedure for micro-strip-based unidirectional UWB antennas," Progress in Electromagnetics Research, vol. 143, pp. 105-130, 2013.

[148] S. Yan, P. J. Soh, and G. A. E. Vandenbosch, "Wearable dualband composite right/ left-handed waveguide textile antenna for WLAN applications," Electronics Letters, vol. 50, no. 6, pp. 424-426, 2014.

[149] H. R. Khaleel, "Design and fabrication of compact inkjet printed antennas for integration within flexible and wearable electronics," IEEE Transactions on Components, Packaging and Manufacturing Technology, vol. 4, no. 10, pp. 1722-1728, 2014.

[150] G. Q. Luo, Z. F. Hu, W. J. Li, X. H. Zhang, L. L. Sun, and J. F. Zheng, "Bandwidth-enhanced low-profile cavity-backed slot antenna by using hybrid SIW cavity modes," IEEE Transactions on Antennas and Propagation, vol. 60, no. 4, pp. 1698-1704, 2012.

[151] D.-F. Guan, Z.-P. Qian, Y.-S. Zhang, and J. Jin, "High-gain SIW cavity-backed array antenna with wideband and low sidelobe characteristics," IEEE Antennas and Wireless Propagation Letters, vol. 14, pp. 1774-1777, 2015.

[152] P. Wu, S. Liao, and Q. Xue, "A substrate integrated slot antenna array using simplified feeding network based on higher order cavity modes," IEEE Transactions on Antennas and Propagation, vol. 64, no. 1, pp. 126-135, 2016.

[153] Q. Wu, H. Wang, C. Yu, and W. Hong, "Low-profile circularly polarized cavity-backed antennas using SIW techniques," IEEE Transactions on Antennas and Propagation, vol. 64, no. 7, pp. 2832-2839, 2016.

[154] O. Caytan, S. Lemey, S. Agneessens et al., "Half-mode substrateintegrated-waveguide cavity-backed slot antenna on cork substrate," IEEE Antennas and Wireless Propagation Letters, vol. 15, pp. 162-165, 2016.

[155] O. Ishihara, T. Mori, H. Sawano, and M. Nakatani, "A highly stabilized GaAs FET oscillator using a dielectric resonator feedback circuit in 9-14 GHz," IEEE Transactions on Microwave Theory and Techniques, vol. 28, no. 8, pp. 817-824, 1980.

[156] S. B. Cohn, "Microwave bandpass filters containing high-Q dielectric resonators," IEEE Transactions on Microwave Theory and Techniques, vol. 16, no. 4, pp. 218-227, 1968.

[157] S. J. Ha, Y. D. Lee, Y. H. Kim, J. J. Choi, and U. S. Hong, "Dielectric resonator oscillator with balanced low noise amplifier," Electronics Letters, vol. 38, no. 24, pp. 1542-1544, 2002.

[158] S. A. Long, M. W. McAllister, and L. C. Shen, "The resonant cylindrical dielectric cavity antenna," IEEE Transactions on Antennas and Propagation, vol. 31, no. 3, pp. 406-412, 1983.

[159] M. W. McAllister, S. A. Long, and G. L. Conway, "Rectangular dielectric resonator antenna," Electronics Letters, vol. 19, no. 6, pp. 218-219, 1983.

[160] M. W. Mcallister and S. A. Long, "Resonant hemispherical dielectric antenna," Electronics Letters, vol. 20, no. 16, pp. 657659, 1984

[161] K. W. Leung, K. M. Luk, E. K. N. Yung, and S. Lai, "Characteristics of a low-profile circular disk DR antenna with very high permittivity," Electronics Letters, vol. 31, no. 6, pp. 417-418, 1995.

[162] H. T. Hui, E. K. N. Yung, and K. W. Leung, "Numerical and experimental studies of a helical antenna loaded by a dielectric resonator," Radio Science, vol. 32, no. 2, pp. 295-304, 1997.

[163] S. M. Shum and K. M. Luk, "Analysis of aperture coupled rectangular dielectric resonator antenna," Electronics Letters, vol. 30, no. 21, pp. 1726-1727, 1994.
[164] G. P. Junker, A. A. Kishk, and A. W. Glisson, "Input impedance of dielectric resonator antennas excited by a coaxial probe," IEEE Transactions on Antennas and Propagation, vol. 42, no. 7, pp. 960-966, 1994.

[165] A. A. Kishk, M. Cuhaci, A. Ittipiboon, and Y. M. M. Antar, "Slot excitation of the dielectric disk radiator," IEEE Transactions on Antennas and Propagation, vol. 43, no. 2, pp. 198-201, 1995.

[166] G. P. Junker, A. A. Kishk, and A. W. Glisson, "Numerical analysis of dielectric resonator antennas excited in quasi-TE modes," Electronics Letters, vol. 29, no. 21, pp. 1810-1811, 1993.

[167] I. A. Eshrah, A. A. Kishk, A. B. Yakovlev, and A. W. Glisson, "Theory and implementation of dielectric resonator antenna excited by a waveguide slot," IEEE Transactions on Antennas and Propagation, vol. 53, no. 1, pp. 483-494, 2005.

[168] R. K. Mongia, A. Ittipiboon, P. Bhartia, and M. Cuhaci, "Electric-monopole antenna using a dielectric ring resonator," Electronics Letters, vol. 29, no. 17, pp. 1530-1531, 1993.

[169] A. Ittipiboon, R. K. Mongia, Y. M. M. Antar, P. Bhartia, and M. Cuhaci, "Aperture fed rectangular and triangular dielectric resonators for use as magnetic dipole antennas," Electronics Letters, vol. 29, no. 23, pp. 2001-2002, 1993.

[170] R. K. Mongia and P. Bhartia, "Dielectric resonator antennas-a review and general design relations for resonant frequency and bandwidth," International Journal of Microwave and MillimeterWave Computer-Aided Engineering, vol. 4, no. 3, pp. 230-247, 1994.

[171] A. B. Kakade and B. Ghosh, "Analysis of the rectangular waveguide slot coupled multilayer hemispherical dielectric resonator antenna," IET Microwaves, Antennas and Propagation, vol. 6, no. 3, pp. 338-347, 2012.

[172] P. M. Jasmine, P. Abdulla, and P. M. Raphika, "Rectangular waveguide fed DRA using tapered waveguide section," Microwave and Optical Technology Letters, vol. 57, no. 9, pp. 2025-2028, 2015.

[173] X.-Q. Sheng, K. W. Leung, and E. K.-N. Yung, "Analysis of waveguide-fed dielectric resonator antenna using a hybrid finite element method/moment method," IEE Proceedings: Microwaves, Antennas and Propagation, vol. 151, no. 1, pp. 9195, 2004

[174] A. Perron, T. A. Denidni, and A. R. Sebak, "Circularly polarized microstrip/elliptical dielectric ring resonator antenna for millimeter-wave applications," IEEE Antennas and Wireless Propagation Letters, vol. 9, pp. 783-786, 2010.

[175] O. G. Avădănei, M. G. Banciu, I. Nicolaescu, and L. Nedelcu, "Superior modes in high permittivity cylindrical dielectric resonator antenna excited by a central rectangular slot," IEEE Transactions on Antennas and Propagation, vol. 60, no. 11, pp. 5032-5038, 2012.

[176] M. Zou, J. Pan, and Z. Nie, "A wideband circularly polarized rectangular dielectric resonator antenna excited by an archimedean spiral slot," IEEE Antennas and Wireless Propagation Letters, vol. 14, pp. 446-449, 2015.

[177] D. Hou, Y.-Z. Xiong, W.-L. Goh, S. Hu, W. Hong, and M. Madihian, "130-GHz on-chip meander slot antennas with stacked dielectric resonators in standard CMOS technology," IEEE Transactions on Antennas and Propagation, vol. 60, no. 9, pp. 4102-4109, 2012.

[178] D. Hou, W. Hong, W.-L. Goh et al., "D-band on-chip higherorder-mode dielectric-resonator antennas fed by half-mode cavity in CMOS technology," IEEE Antennas and Propagation Magazine, vol. 56, no. 3, pp. 80-89, 2014. 
[179] A. Rashidian, L. Shafai, and D. M. Klymyshyn, "Tall microstrip transmission lines for dielectric resonator antenna applications," IET Microwaves, Antennas and Propagation, vol. 8, no. 2, pp. 112-124, 2014.

[180] A. Rashidian and D. M. Klymyshyn, "Compact wideband multimode dielectric resonator antennas fed with parallel standing strips," IEEE Transactions on Antennas and Propagation, vol. 60, no. 11, pp. 5021-5031, 2012.

[181] M. A. Saed and R. Yadla, "Microstrip-fed low profile and compact dielectric resonator antennas," Progress in Electromagnetics Research, vol. 56, pp. 151-162, 2006.

[182] L. Zou and C. Fumeaux, "A cross-shaped dielectric resonator antenna for multifunction and polarization diversity applications," IEEE Antennas and Wireless Propagation Letters, vol. 10, pp. 742-745, 2011.

[183] M. Simeoni, R. Cicchetti, A. Yarovoy, and D. Caratelli, "Plasticbased supershaped dielectric resonator antennas for wide-band applications," IEEE Transactions on Antennas and Propagation, vol. 59, no. 12, pp. 4820-4825, 2011.

[184] D. Guha, A. Banerjee, C. Kumar, and Y. M. M. Antar, "New technique to excite higher-order radiating mode in a cylindrical dielectric resonator antenna," IEEE Antennas and Wireless Propagation Letters, vol. 13, pp. 15-18, 2014.

[185] R. Cicchetti, A. Faraone, E. Miozzi, R. Ravanelli, and O. Testa, "A high-gain mushroom-shaped dielectric resonator antenna for wideband wireless applications," IEEE Transactions on Antennas and Propagation, vol. 64, no. 7, pp. 2848-2861, 2016.

[186] B. Mukherjee, P. Patel, and J. Mukherjee, "A novel cup-shaped inverted hemispherical dielectric resonator antenna for wideband applications," IEEE Antennas and Wireless Propagation Letters, vol. 12, pp. 1240-1243, 2013.

[187] R. Cicchetti, E. Miozzi, and O. Testa, "A novel wideband multipermittivity composite dielectric resonator antenna for wireless applications," in Proceedings of the IEEE-APS Topical Conference on Antennas and Propagation in Wireless Communications (APWC '16), pp. 70-73, Cairns, Australia, September 2016.

[188] Y. Gao, Z. Feng, and L. Zhang, "Compact asymmetrical Tshaped dielectric resonator antenna for broadband applications," IEEE Transactions on Antennas and Propagation, vol. 60, no. 3, pp. 1611-1615, 2012.

[189] B. Li and K. W. Leung, "Strip-fed rectangular dielectric resonator antennas with/without a parasitic patch," IEEE Transactions on Antennas and Propagation, vol. 53, no. 7, pp. 2200-2207, 2005.

[190] A. S. Al-Zoubi and A. A. Kishk, "Wide band strip-fed rectangular dielectric resonator antenna," in Proceedings of the $3 \mathrm{rd}$ European Conference on Antennas and Propagation (EuCAP '09), pp. 2379-2382, Berlin, Germany, March 2009.

[191] D. Soren, R. Ghatak, R. K. Mishra, and D. R. Poddar, "Dielectric resonator antennas: designs and advances," Progress in Electromagnetics Research B, vol. 60, no. 1, pp. 195-213, 2014.

[192] M. Simeoni, R. Cicchetti, A. Yarovoy, and D. Caratelli, "Supershaped dielectric resonator antennas," in Proceedings of the IEEE International Symposium on Antennas and Propagation and USNC/URSI National Radio Science Meeting (APSURSI '09), June 2009.

[193] M. Simeoni, R. Cicchetti, A. Yarovoy, and D. Caratelli, "Circularly polarized supershaped dielectric resonator antennas for indoor ultra wide band applications," in Proceedings of the IEEE International Symposium on Antennas and Propagation and CNC-USNC/URSI Radio Science Meeting-Leading the Wave (AP-S/URSI '10), pp. 1-4, Ontario, Canada, July 2010.
[194] Y. Wang, T. A. Denidni, Q. Zeng, and G. Wei, "A wideband highgain stacked cylindrical dielectric resonator antenna," Progress in Electromagnetics Research Letters, vol. 43, pp. 155-163, 2013.

[195] Y. M. Pan and K. W. Leung, "Wideband omnidirectional circularly polarized dielectric resonator antenna with parasitic strips," IEEE Transactions on Antennas and Propagation, vol. 60, no. 6, pp. 2992-2997, 2012.

[196] M. Abedian, S. K. A. Rahim, S. Danesh, S. Hakimi, L. Y. Cheong, and M. H. Jamaluddin, "Novel design of compact UWB dielectric resonator antenna with dual-band-rejection characteristics for WiMAX/WLAN bands," IEEE Antennas and Wireless Propagation Letters, vol. 14, pp. 245-248, 2015.

[197] X. Li, Y. Yang, F. Gao, H. Ma, and X. Shi, "A compact dielectric resonator antenna excited by a planar monopole patch for wideband applications," International Journal of Antennas and Propagation, vol. 2016, Article ID 9734781, 9 pages, 2016.

[198] Z. N. Chen, X. Qing, T. S. P. See, and W. K. Toh, "Antennas for WiFi connectivity," Proceedings of the IEEE, vol. 100, no. 7, pp. 2322-2329, 2012.

[199] S. Gao, K. Clark, M. Unwin et al., "Antennas for modern small satellites," IEEE Antennas and Propagation Magazine, vol. 51, no. 4, pp. 40-56, 2009.

[200] A. Perron, T. A. Denidni, and A.-R. Sebak, "High-gain hybrid dielectric resonator antenna for millimeter-wave applications: design and implementation," IEEE Transactions on Antennas and Propagation, vol. 57, no. 10, pp. 2882-2892, 2009.

[201] D. Guha, A. Banerjee, C. Kumar, and Y. M. M. Antar, "Higher order mode excitation for high-gain broadside radiation from cylindrical dielectric resonator antennas," IEEE Transactions on Antennas and Propagation, vol. 60, no. 1, pp. 71-77, 2012.

[202] A. Petosa and S. Thirakoune, "Rectangular dielectric resonator antennas with enhanced gain," IEEE Transactions on Antennas and Propagation, vol. 59, no. 4, pp. 1385-1389, 2011.

[203] Y. Ge, K. P. Esselle, and T. S. Bird, "Compact dielectric resonator antennas with ultrawide $60 \%-110 \%$ bandwidth," IEEE Transactions on Antennas and Propagation, vol. 59, no. 9, pp. 3445-3448, 2011.

[204] Skyworks Solutions, Inc., 2015, http://www.skyworksinc.com/.

[205] MACOM, 2015, http://www.macom.com/.

[206] G.-P. Gao, X. Yang, and J.-S. Zhang, "A printed volcano smoke antenna for UWB and WLAN communications," Progress in Electromagnetics Research Letters, vol. 4, pp. 55-61, 2008.

[207] R. Azim, M. T. Islam, J. S. Mandeep, and A. T. Mobashsher, "A planar circular ring ultra-wideband antenna with dual bandnotched characteristics," Journal of Electromagnetic Waves and Applications, vol. 26, no. 14-15, pp. 2022-2032, 2012.

[208] F. Zhu, S. Gao, A. T. S. Ho et al., "Multiple band-notched UWB antenna with band-rejected elements integrated in the feed line," IEEE Transactions on Antennas and Propagation, vol. 61, no. 8, pp. 3952-3960, 2013.

[209] P. Gao, S. He, X. Wei, Z. Xu, N. Wang, and Y. Zheng, "Compact printed UWB diversity slot antenna with $5.5-\mathrm{GHz}$ band-notched characteristics," IEEE Antennas and Wireless Propagation Letters, vol. 13, pp. 376-379, 2014.

[210] M. S. A. Rani, S. K. A. Rahim, M. R. Kamarudin, T. Peter, S. W. Cheung, and B. M. Saad, "Electromagnetic behaviors of thin film CPW-Fed CSRR loaded on UWB transparent antenna," IEEE Antennas and Wireless Propagation Letters, vol. 13, pp. 1239-1242, 2014.

[211] N. Jaglan, B. K. Kanaujia, S. D. Gupta, and S. Srivastava, "Triple band notched UWB antenna design using electromagnetic 
band gap structures," Progress In Electromagnetics Research C, vol. 66, pp. 139-147, 2016.

[212] W. Wu, Y. Li, R. Wu, C. Shi, and T. Cui, "Band-notched UWB antenna with switchable and tunable performance," International Journal of Antennas and Propagation, vol. 2016, Article ID 9612987, 6 pages, 2016. 


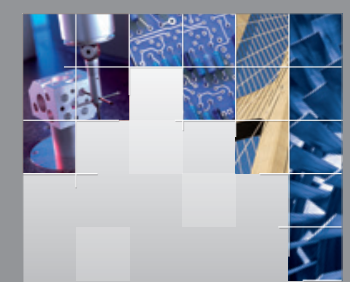

\section{Enfincering}
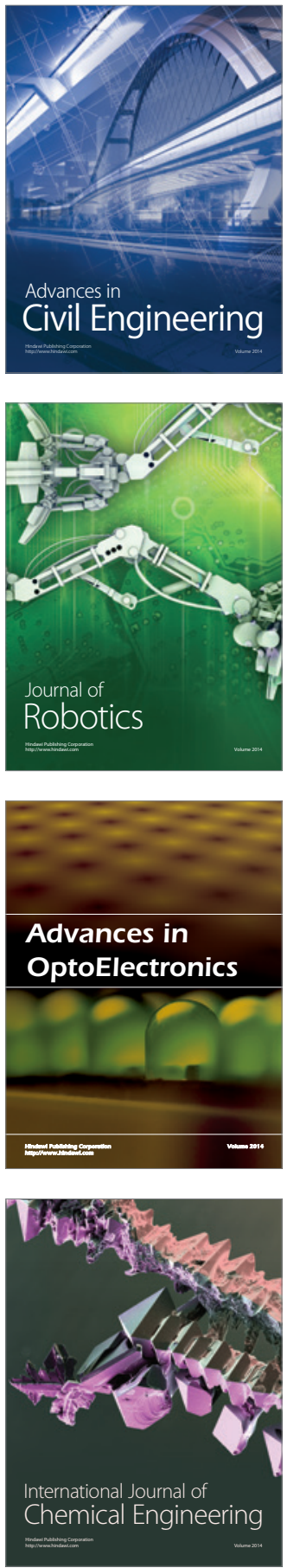

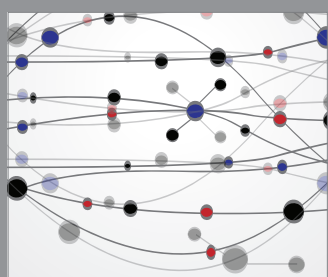

The Scientific World Journal

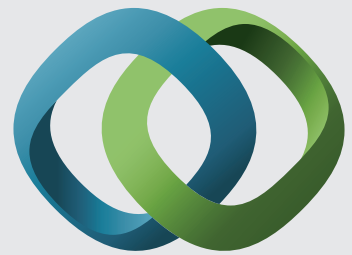

\section{Hindawi}

Submit your manuscripts at

https://www.hindawi.com
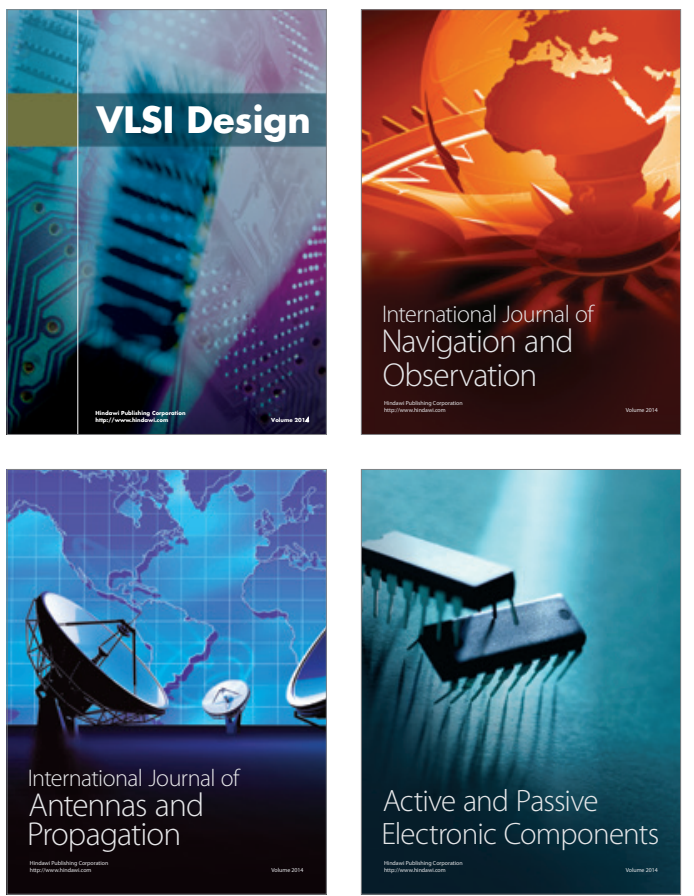
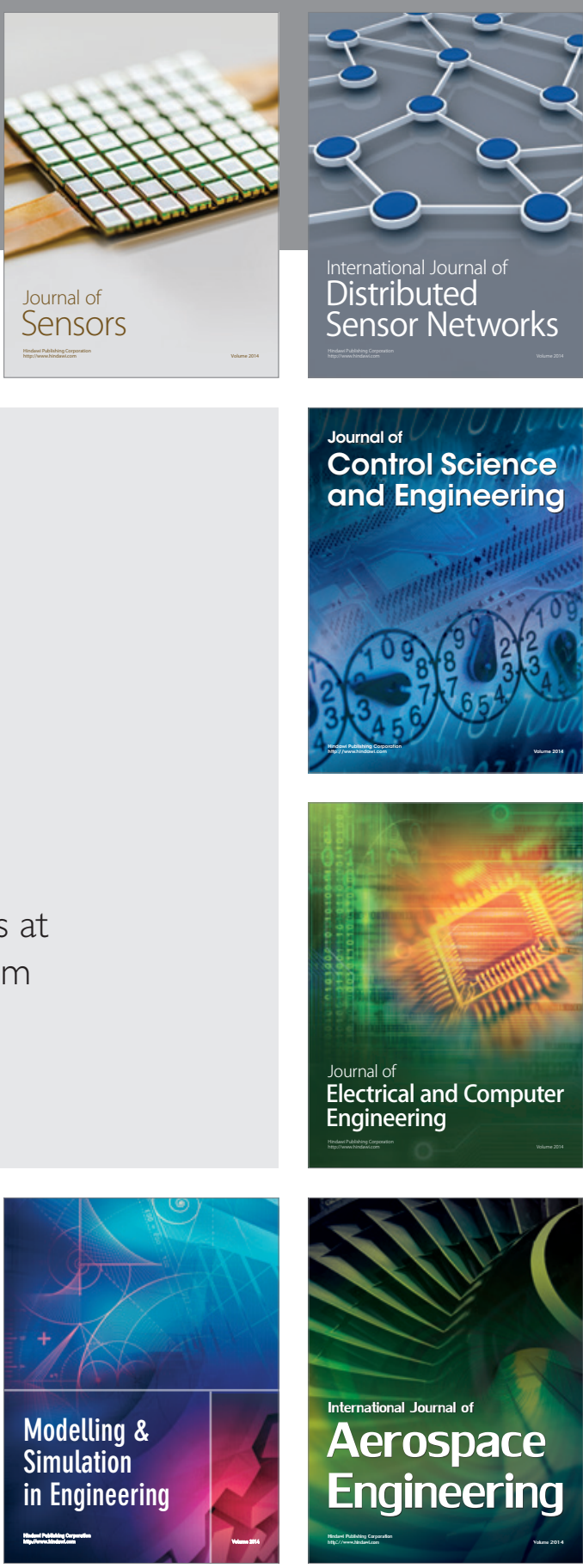

International Journal of

Distributed

Sensor Networks

$-$

Joumal of

Control Science

and Engineering
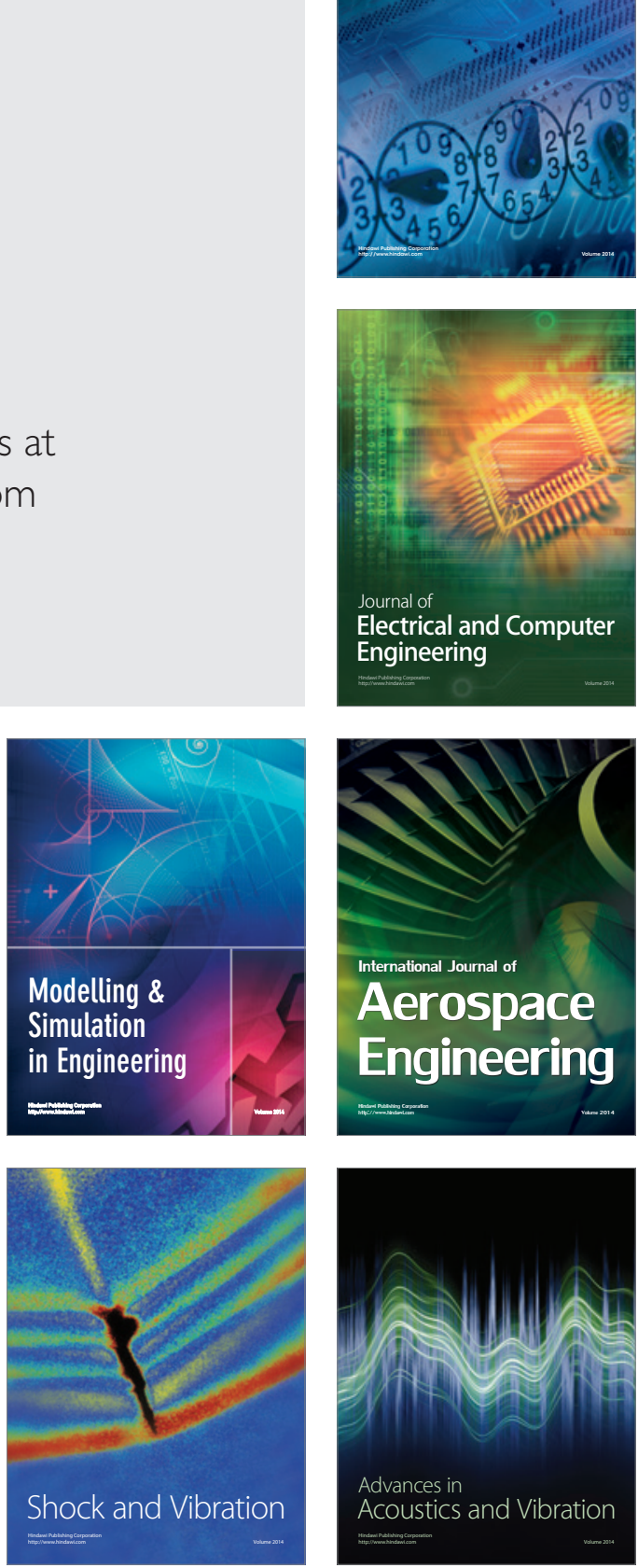